\title{
Enabling and probing oxidative addition and reductive elimination at a Group 14 metal center: cleavage and functionalization of E-H bonds by a bis(boryl)stannylene
}

Andrey V. Protchenko, Joshua I. Bates, Liban M. A. Saleh, Matthew P.

Blake, Andrew D. Schwarz, Eugene L. Kolychev, Amber L. Thompson, Cameron Jones, Philip Mountford and Simon Aldridge

\section{SUPPORTING INFORMATION}

1. Representative NMR spectra for new compounds $\quad$ S2

2. Additional experiments on ammonia activation chemistry $\quad$ S14

$\begin{array}{ll}\text { 3. Additional synthetic data } & \text { S17 }\end{array}$

4. Additional crystallographic data $\quad$ S25

$\begin{array}{ll}\text { 5. DFT run files } & \text { S28 }\end{array}$

(55 pages total) 


\section{Representative NMR spectra for new compounds}

\section{$\mathrm{H}_{2} \operatorname{Sn}\left\{\mathrm{B}(\mathrm{NDippCH})_{2}\right\}_{2}(3)$}
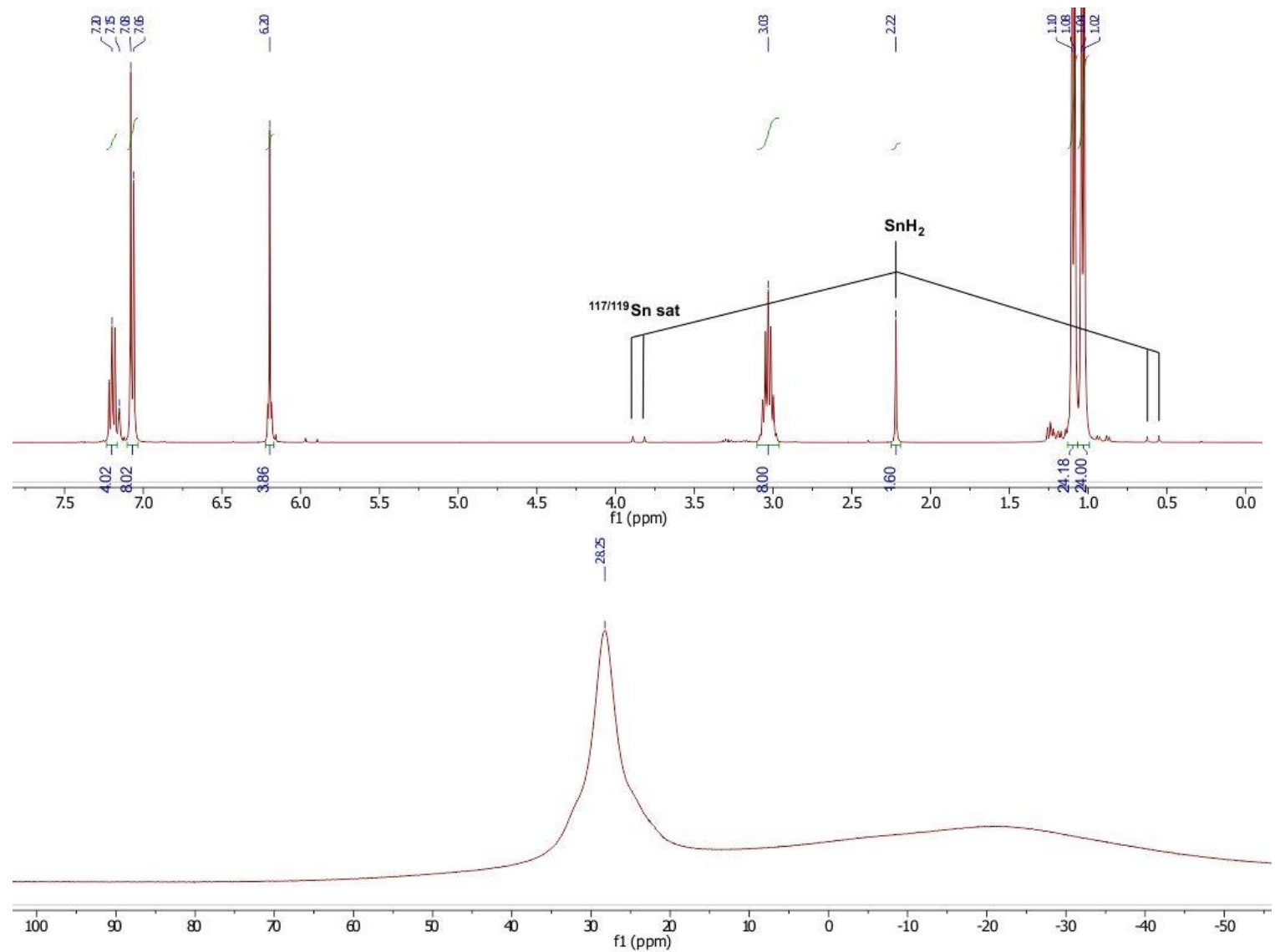

Figure s1: ${ }^{1} \mathrm{H}$ and ${ }^{11} \mathrm{~B}$ NMR spectra of crystalline $\mathrm{Sn}\left\{\mathrm{B}(\mathrm{NDippCH})_{2}\right\}_{2}(\mathrm{H})_{2}(\mathbf{3})$. 


\section{$\left.\operatorname{Sn}\{\text { B(NDippCH })_{2}\right\}_{2}(\mathrm{H})\left(\mathrm{SiH}_{3}\right)(\mathbf{4 a})$}

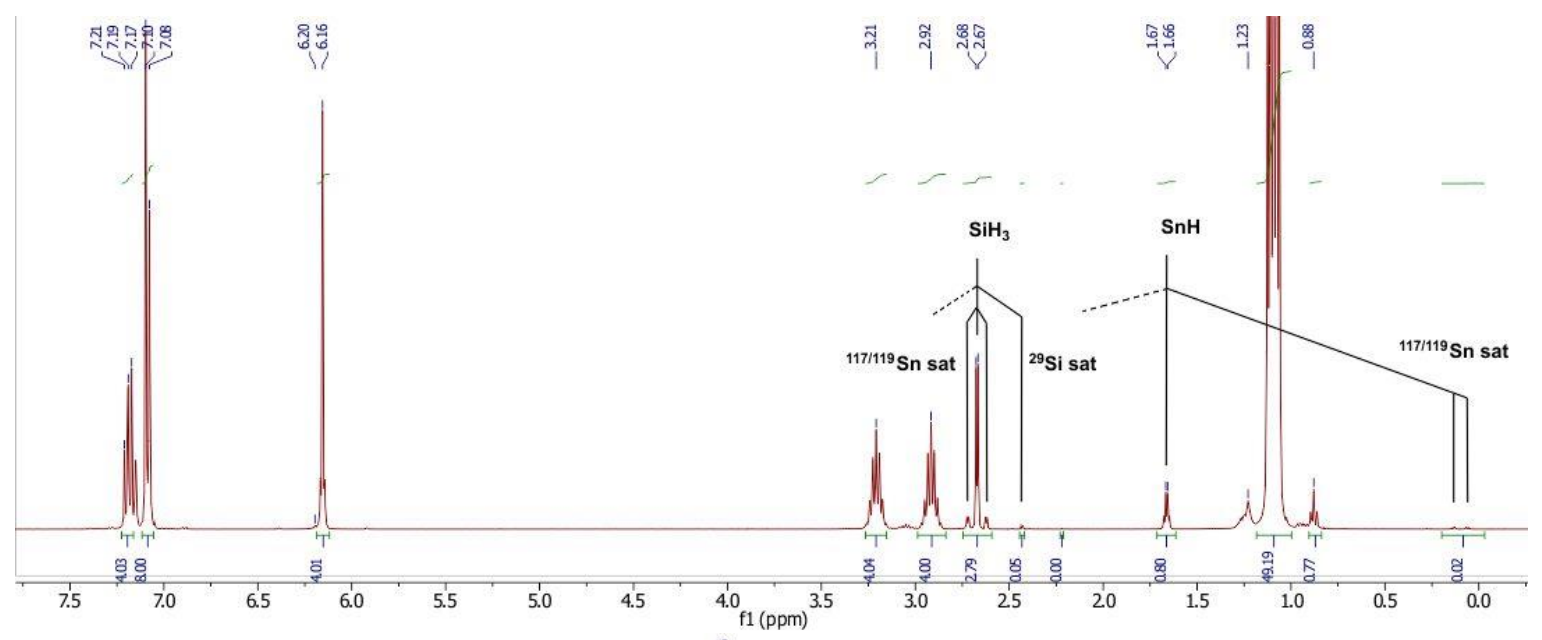

离

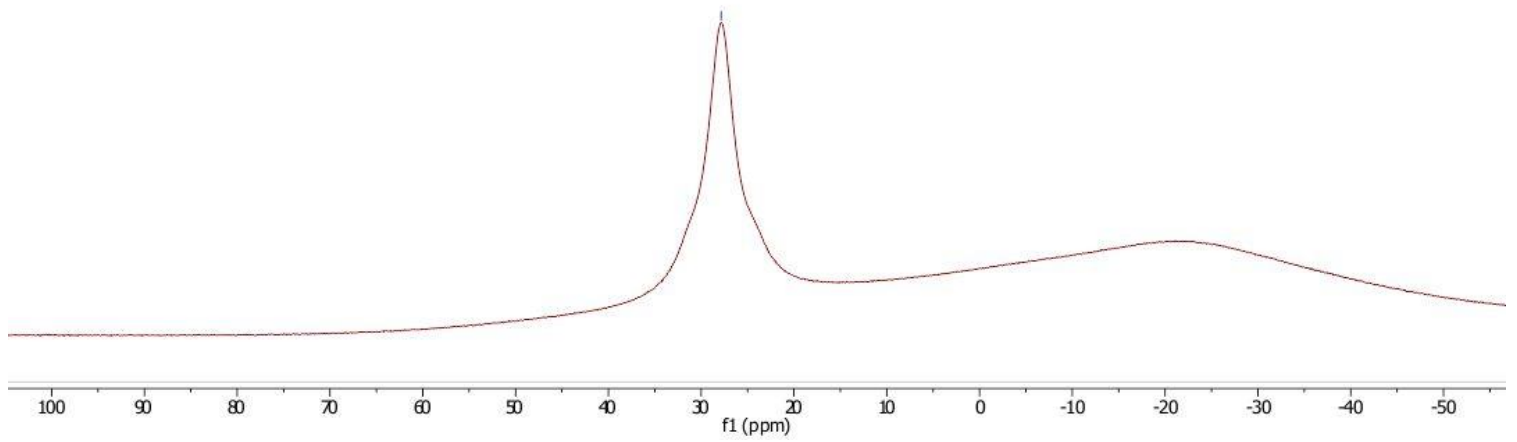

Figure s2: ${ }^{1} \mathrm{H}$ and ${ }^{11} \mathrm{~B}$ NMR spectra of crystalline $\mathrm{Sn}\left\{\mathrm{B}(\mathrm{NDippCH})_{2}\right\}_{2}(\mathrm{H})\left(\mathrm{SiH}_{3}\right)$ (peaks at $\delta 0.88$ and $1.23 \mathrm{ppm}$ belong to residual hexane) (4a) 


\section{$\mathrm{Sn}\left\{\mathrm{B}(\mathrm{NDippCH})_{2}\right\}_{2}(\mathrm{H})\left(\mathrm{SiH}_{2} \mathrm{Ph}\right)(\mathbf{4 b})$}
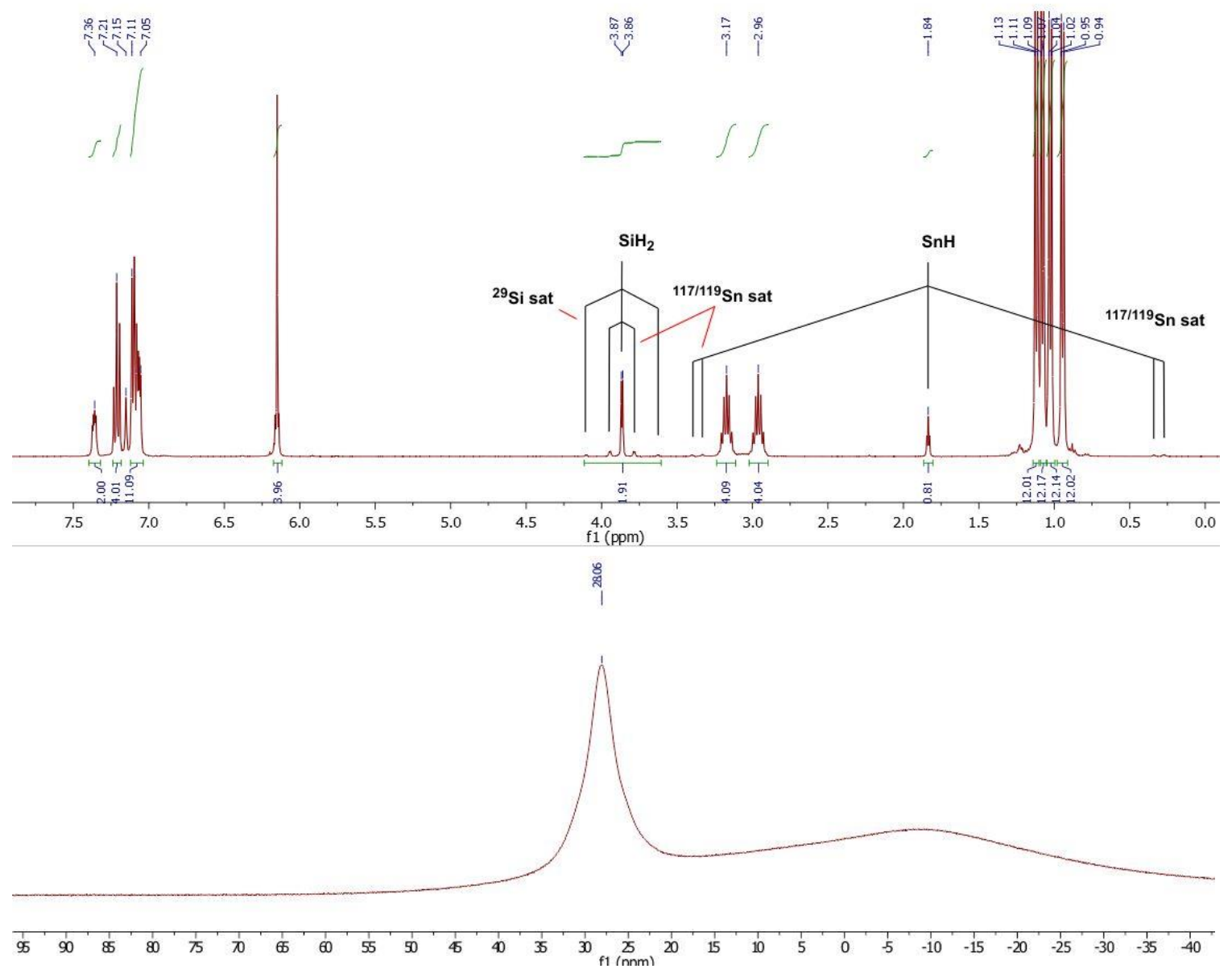

Figure s3: ${ }^{1} \mathrm{H}$ and ${ }^{11} \mathrm{~B}\left\{{ }^{1} \mathrm{H}\right\}$ NMR spectra of crystalline $\mathrm{Sn}\left\{\mathrm{B}(\mathrm{NDippCH})_{2}\right\}_{2}(\mathrm{H})\left(\mathrm{SiH}_{2} \mathrm{Ph}\right)$ (4b) 


\section{$\mathrm{Sn}\left\{\mathrm{B}(\mathrm{NDippCH})_{2}\right\}_{2}(\mathrm{H})\left(\mathrm{BH}_{2} \mathrm{NMe}_{3}\right)(5)$}
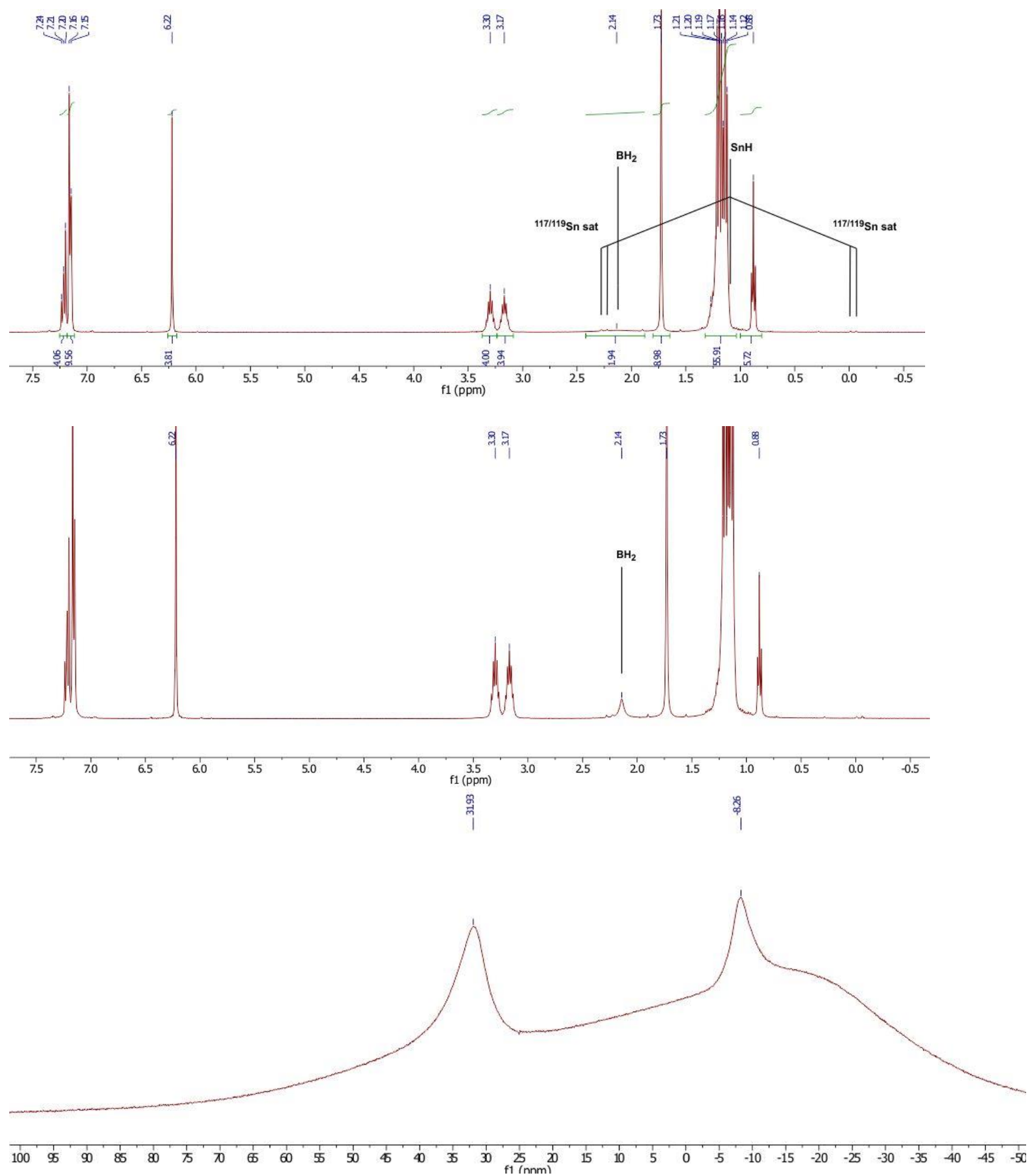

Figure s4: ${ }^{1} \mathrm{H}, \quad{ }^{1} \mathrm{H}\left\{{ }^{11} \mathrm{~B}\right\}$ and ${ }^{11} \mathrm{~B}\left\{{ }^{1} \mathrm{H}\right\} \quad$ NMR spectra of the crystalline $\mathrm{Sn}\left\{\mathrm{B}(\mathrm{NDippCH})_{2}\right\}_{2}(\mathrm{H})\left(\mathrm{BH}_{2} \mathrm{NMe}_{3}\right)(\mathbf{5})$ 
$\operatorname{Sn}\left\{\mathrm{B}(\mathrm{NDippCH})_{2}\right\}_{2}(\mathrm{H})(\mathrm{OH})(6)$
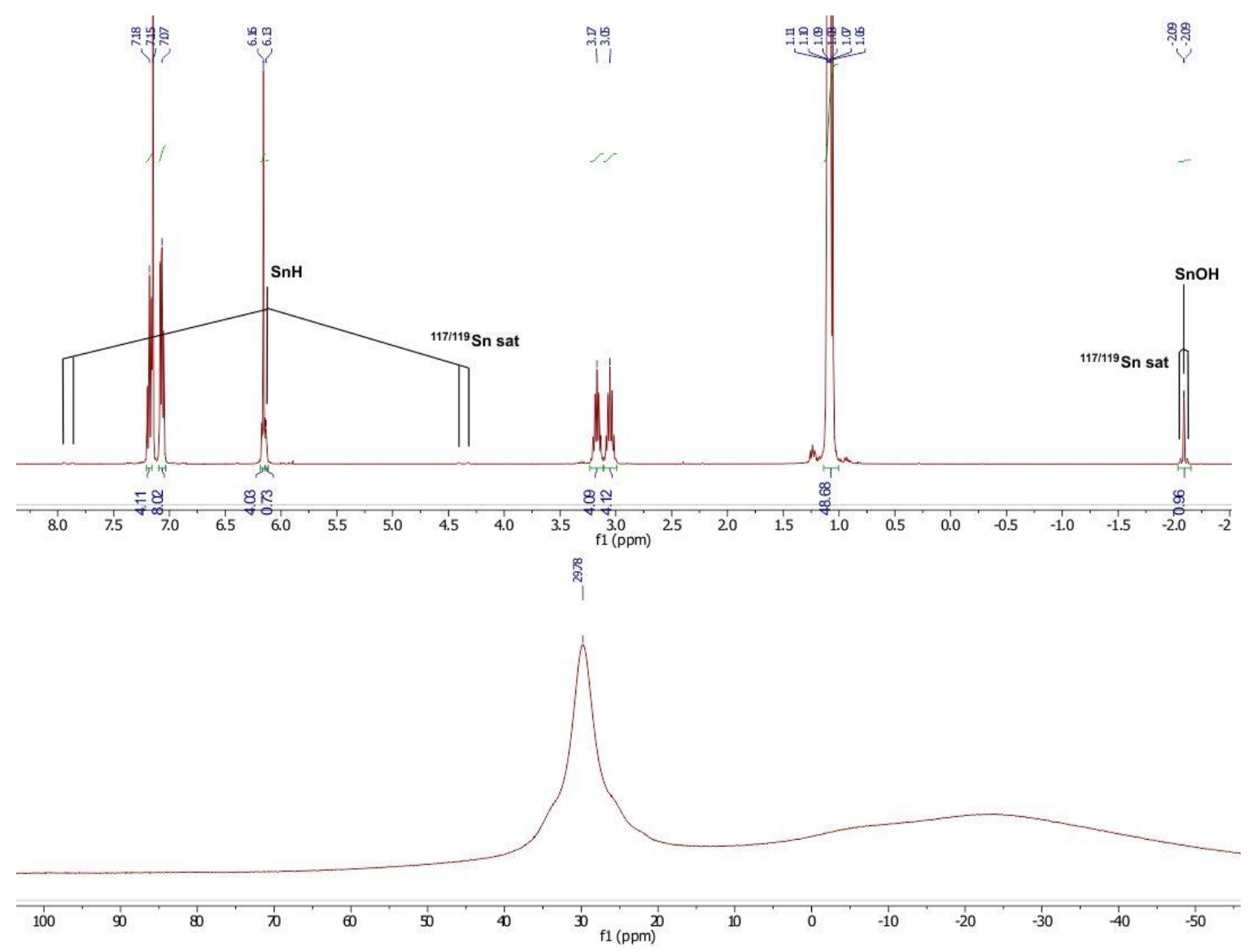

Figure s5: ${ }^{1} \mathrm{H}$ and ${ }^{11} \mathrm{~B}$ NMR spectra of the crystalline $\mathrm{Sn}\left\{\mathrm{B}(\mathrm{NDippCH})_{2}\right\}_{2}(\mathrm{H})(\mathrm{OH})(\mathbf{6})$ 


\section{$\operatorname{Sn}\left\{\mathrm{B}(\mathrm{NDippCH})_{2}\right\}_{2}\left({ }^{t} \mathrm{BuNH}_{2}\right)\left(1^{t} \mathrm{BuNH}_{2}\right)$}

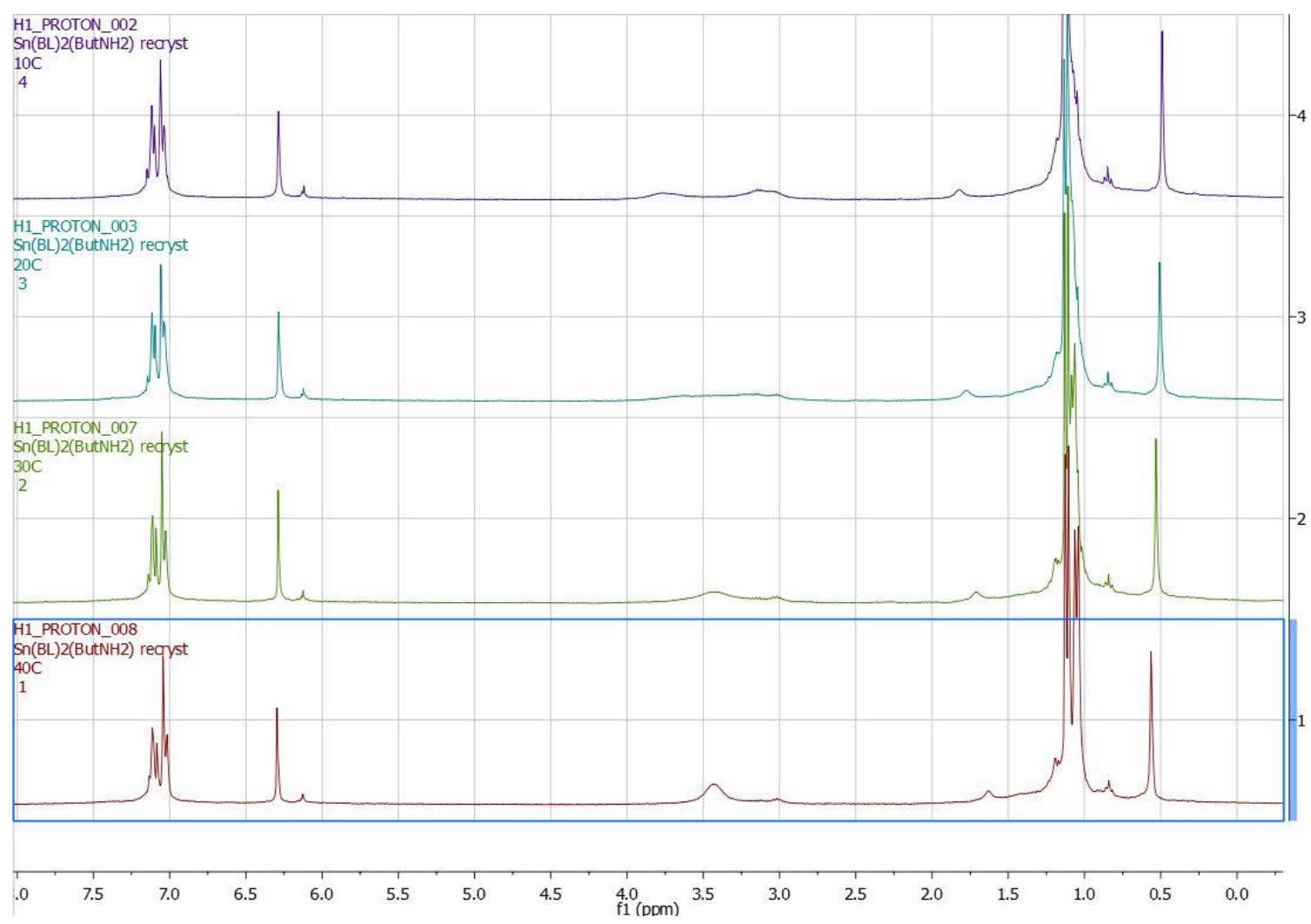

Figure s6: Variable temperature ${ }^{1} \mathrm{H} \quad$ NMR $\quad$ spectra of crystalline $\mathrm{Sn}\left\{\mathrm{B}(\mathrm{NDippCH})_{2}\right\}_{2}\left({ }^{t} \mathrm{BuNH}_{2}\right)\left(\mathbf{1}^{t} \mathrm{BuNH}_{2}\right)$ 


\section{$\operatorname{Sn}\left\{\mathrm{B}(\mathrm{NDippCH})_{2}\right\}_{2}\left(\mathrm{NH}_{3}\right)\left(\mathbf{1} \mathrm{NH}_{3}\right)$}
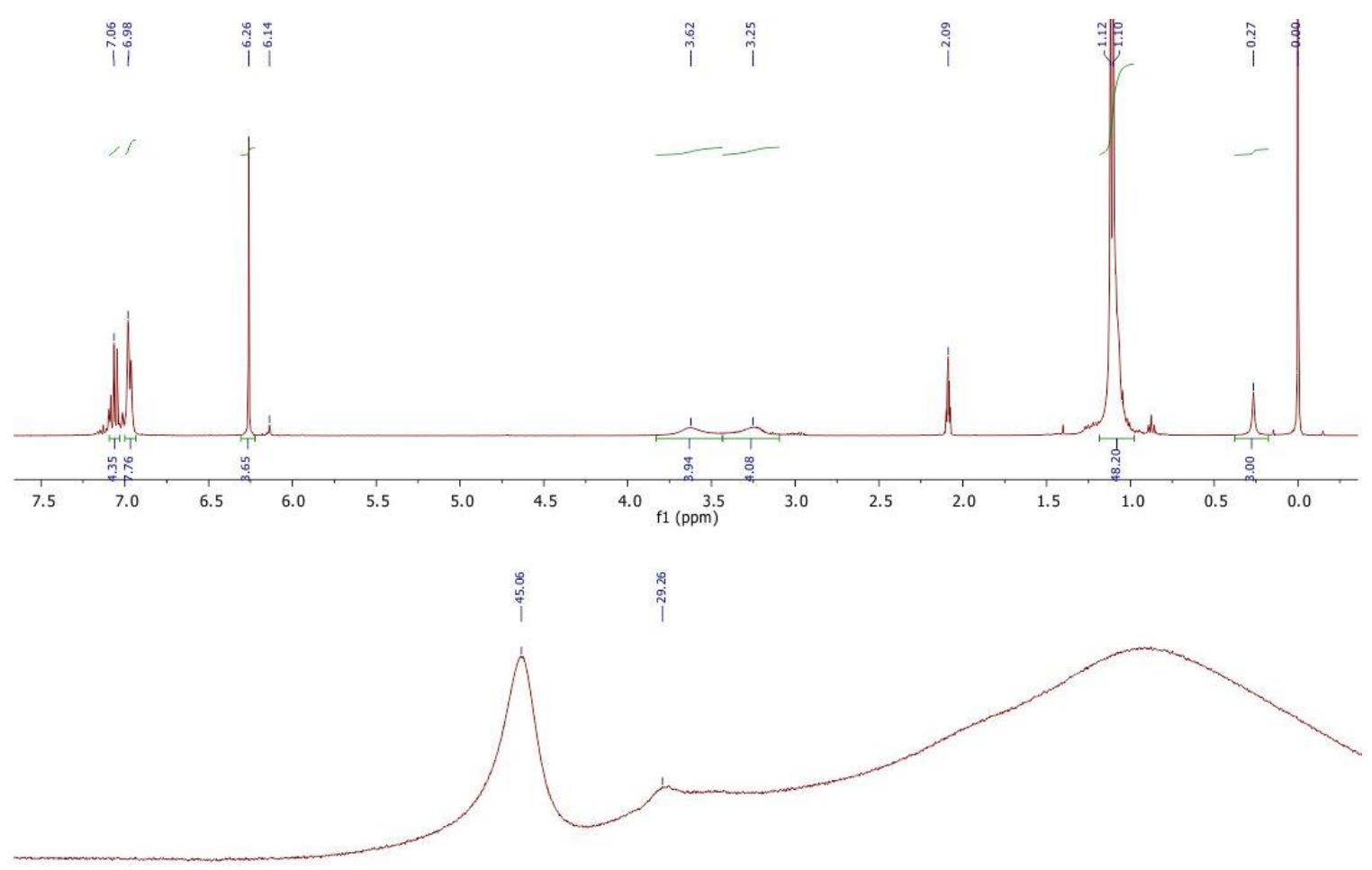

$\begin{array}{lllllllllllllllllllllllllllllllllllllllllll}100 & 95 & 90 & 85 & 80 & 75 & 70 & 65 & 60 & 55 & 50 & 45 & 40 & 35 & 30 & 25 & 20 & 15 & 10 & 5 & 0 & -5 & -10 & -15 & -20 & -25 & -30 & -35 & -40 & -45\end{array}$

Figure s7: ${ }^{1} \mathrm{H}$ and ${ }^{11} \mathrm{~B}$ NMR $\left(\mathrm{C}_{6} \mathrm{D}_{5} \mathrm{CD}_{3}\right)$ spectra of crystalline $\mathrm{Sn}\left\{\mathrm{B}(\mathrm{NDippCH})_{2}\right\}_{2}\left(\mathrm{NH}_{3}\right)$ $\left(1 \cdot \mathrm{NH}_{3}\right)$ 


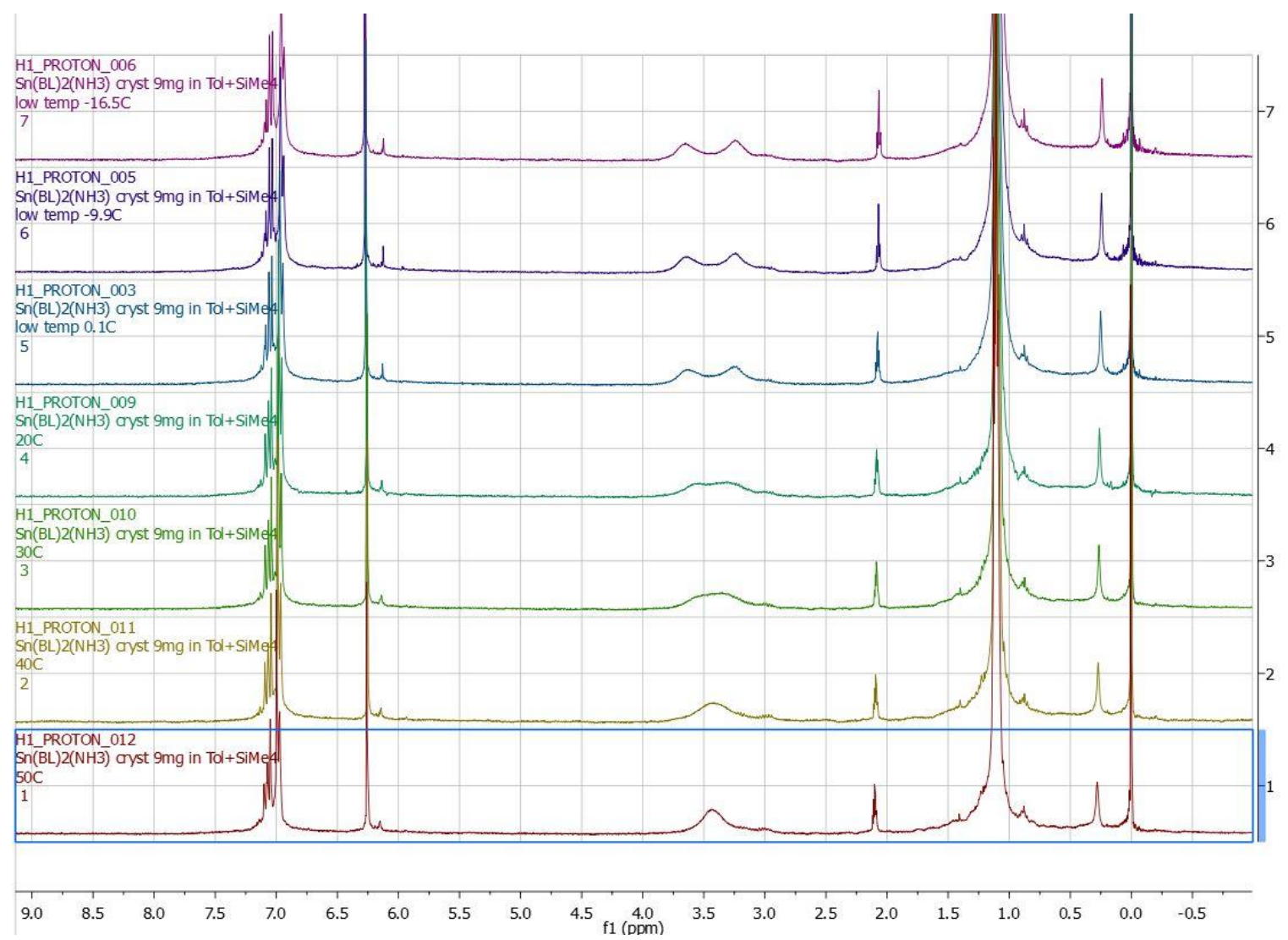

Figure s8: $\quad$ Variable temperature ${ }^{1} \mathrm{H} \quad$ NMR $\quad$ spectra of crystalline $\mathrm{Sn}\left\{\mathrm{B}(\mathrm{NDippCH})_{2}\right\}_{2}\left(\mathrm{NH}_{3}\right)\left(\mathbf{1} \cdot \mathrm{NH}_{3}\right)$ 


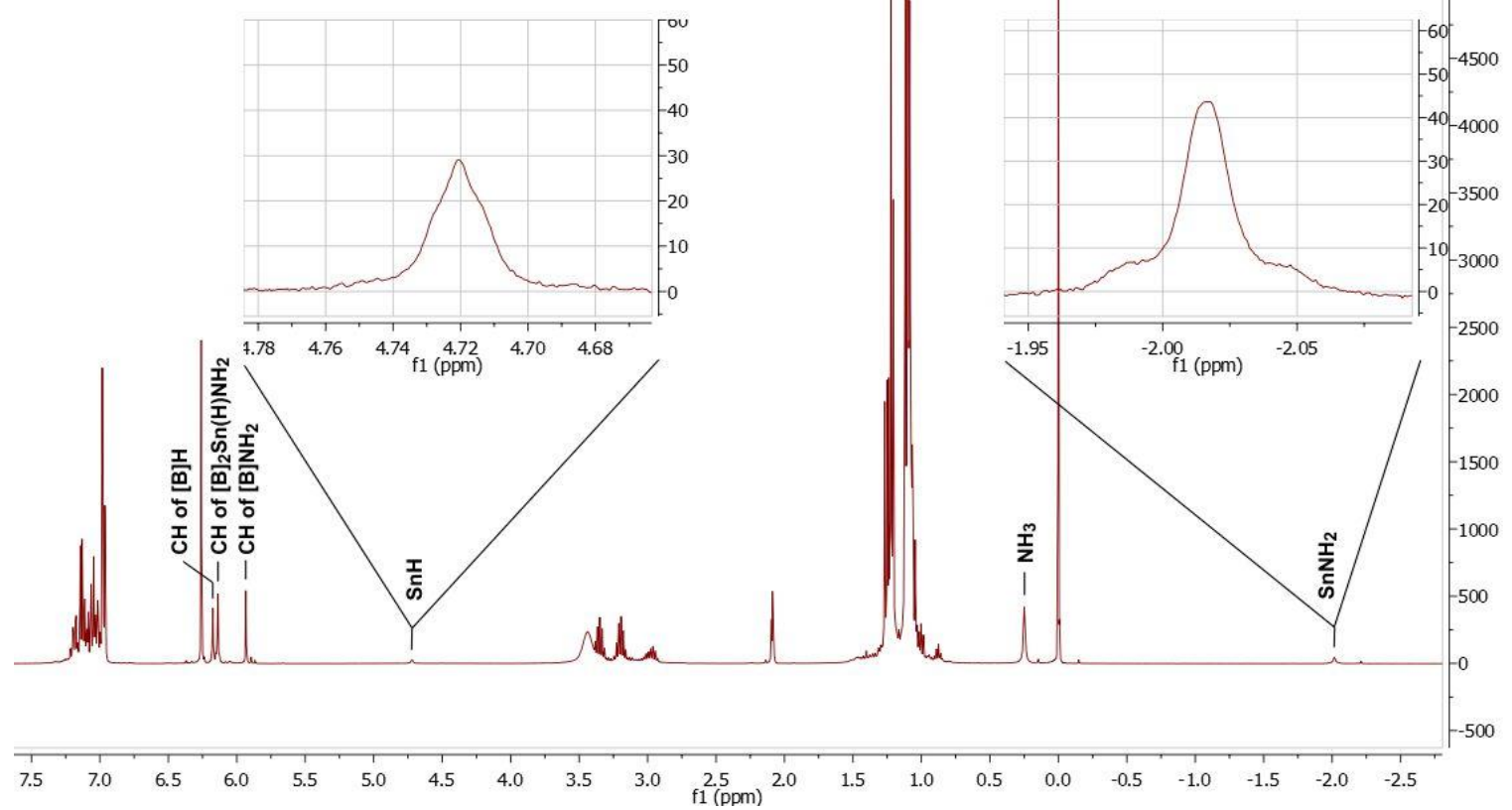

Figure s9: ${ }^{1} \mathrm{H} \mathrm{NMR}\left(\mathrm{C}_{6} \mathrm{D}_{5} \mathrm{CD}_{3}\right)$ spectrum of $\mathrm{Sn}\left\{\mathrm{B}(\mathrm{NDippCH})_{2}\right\}_{2}\left(\mathrm{NH}_{3}\right)$ after $2 \mathrm{~d}$ at $\mathrm{RT}$

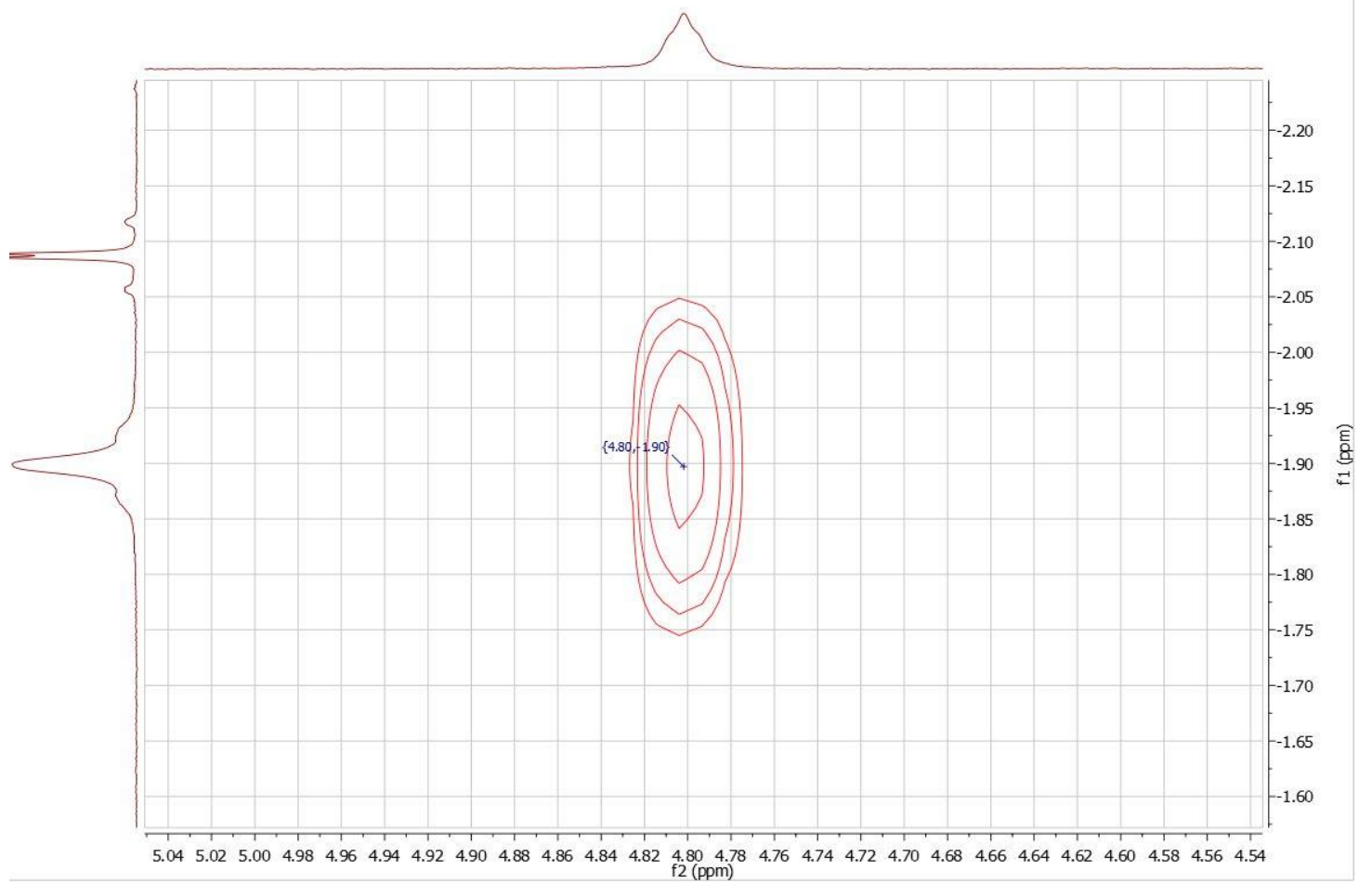




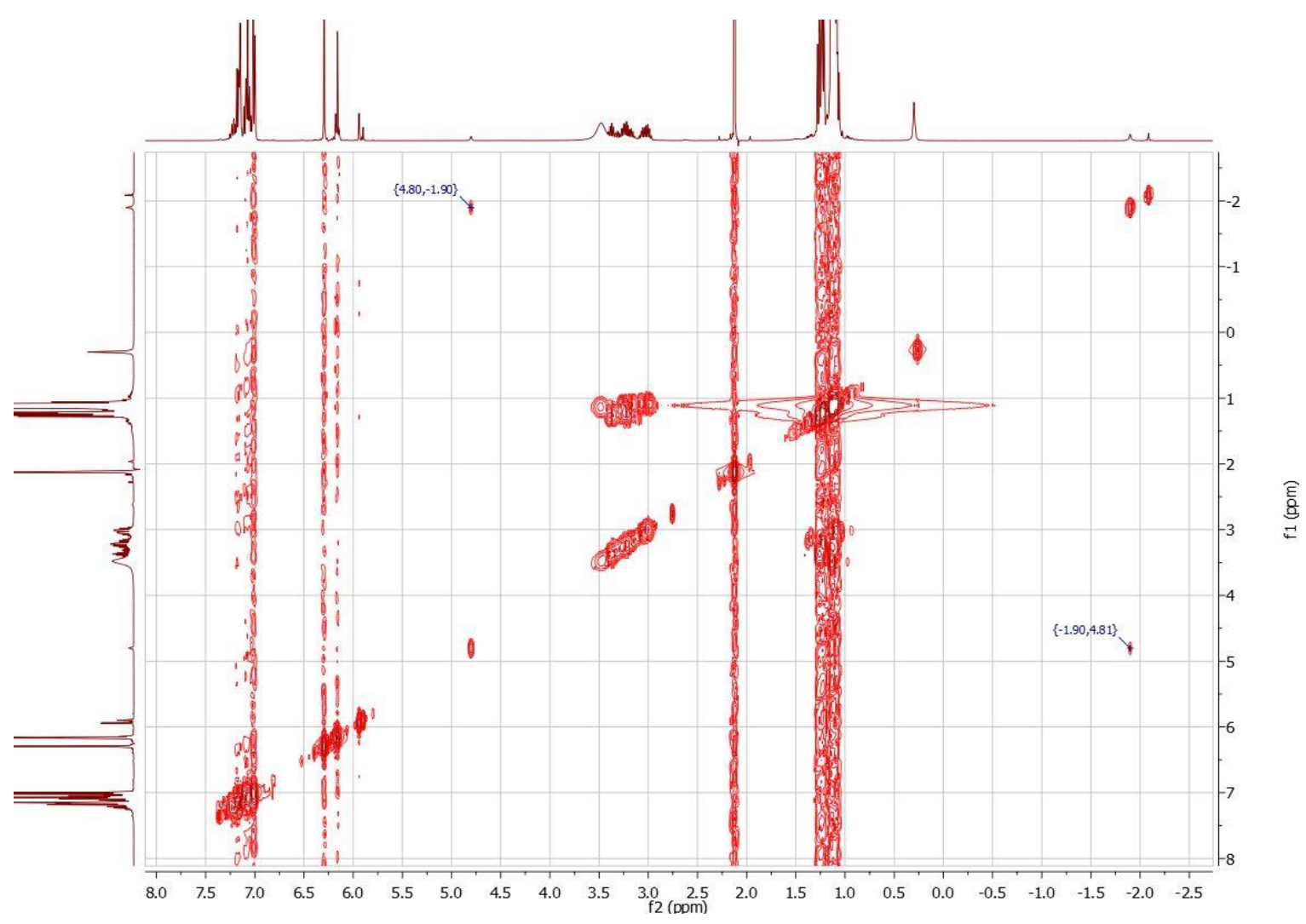

Figure s10: ${ }^{1} \mathrm{H} /{ }^{1} \mathrm{H}$ COSY NMR $\left(\mathrm{C}_{6} \mathrm{D}_{6}\right)$ spectrum of $\mathrm{Sn}\left\{\mathrm{B}(\mathrm{NDippCH})_{2}\right\}_{2}\left(\mathrm{NH}_{3}\right)$ after $2 \mathrm{~d}$ at $20{ }^{\circ} \mathrm{C}$ showing cross-peak for $\mathrm{SnH} / \mathrm{SnNH}_{2}$ unresolved coupling

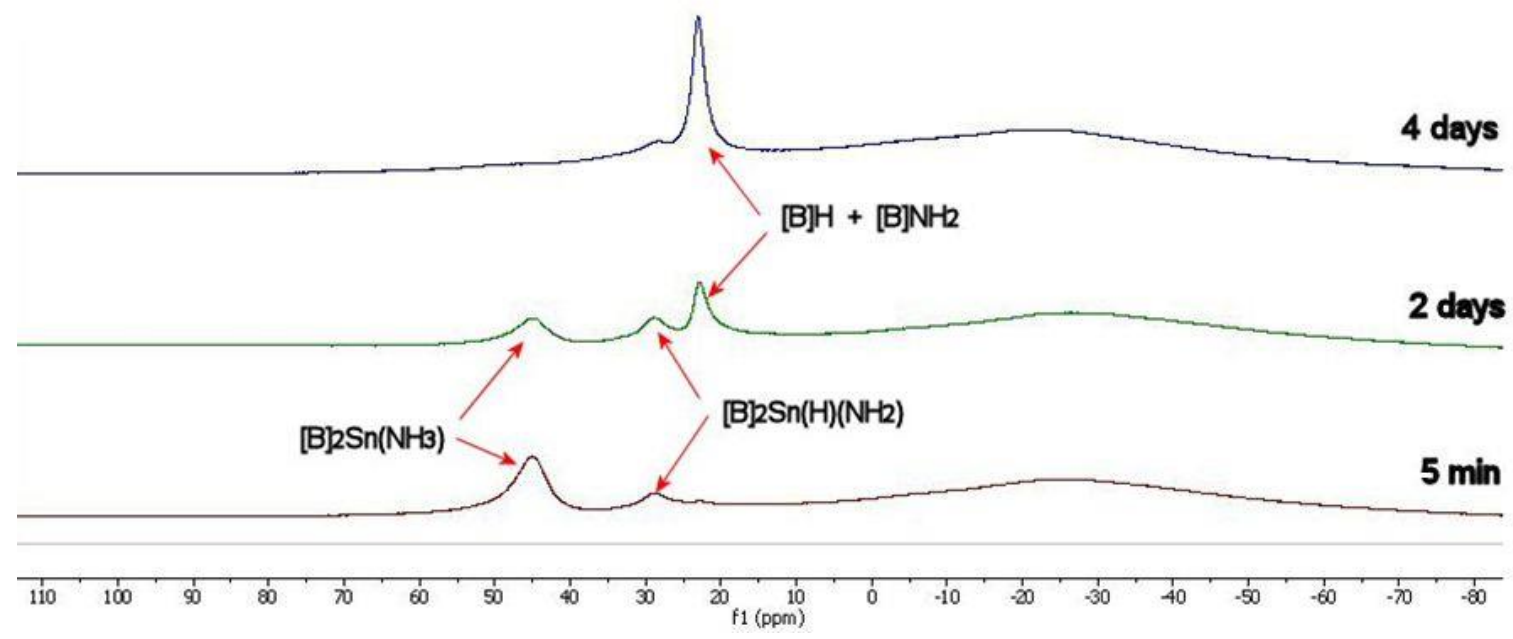

Figure s11: ${ }^{11} \mathrm{~B}$ NMR $\left(\mathrm{C}_{6} \mathrm{D}_{6}\right)$ spectrum of $\mathrm{Sn}\left\{\mathrm{B}(\mathrm{NDippCH})_{2}\right\}_{2}\left(\mathrm{NH}_{3}\right)$ after $5 \mathrm{~min}, 2 \mathrm{~d}$ and $4 \mathrm{~d}$ at RT 


\section{$\operatorname{Sn}\left\{\mathrm{B}(\mathrm{NDippCH})_{2}\right\}_{2}(\mathrm{H})\left(\mathrm{NH}_{2}\right)(\mathbf{7})$}
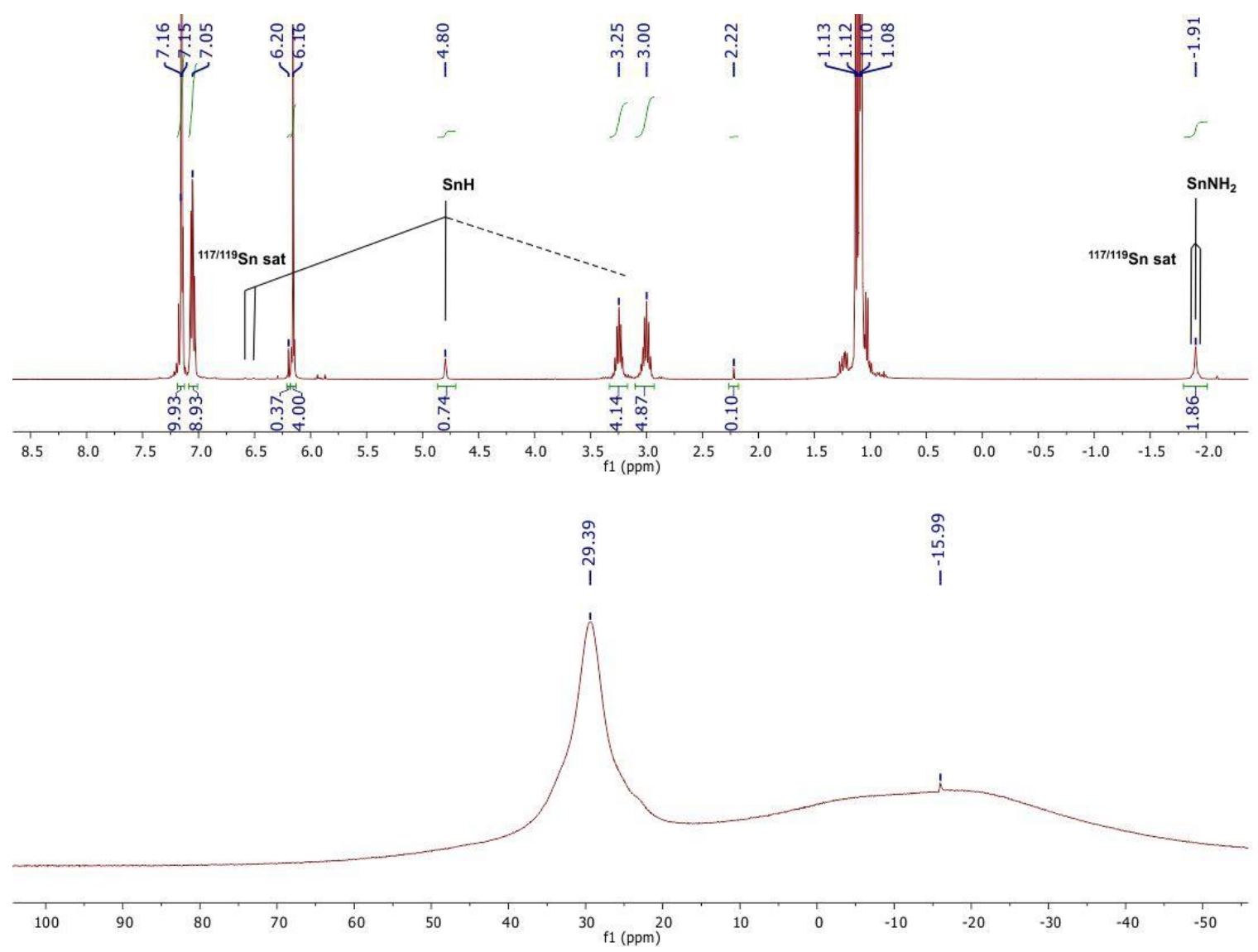

Figure s12: ${ }^{1} \mathrm{H}$ and ${ }^{11} \mathrm{~B}$ NMR spectra of crystalline $\mathrm{Sn}\left\{\mathrm{B}(\mathrm{NDippCH})_{2}\right\}_{2}(\mathrm{H})\left(\mathrm{NH}_{2}\right)(7)$ 
$\left[\mathrm{Sn}\left\{\mathrm{B}(\mathrm{NDippCH})_{2}\right\}\left(\mu-\mathrm{NH}_{2}\right)\right]_{2}(8)$
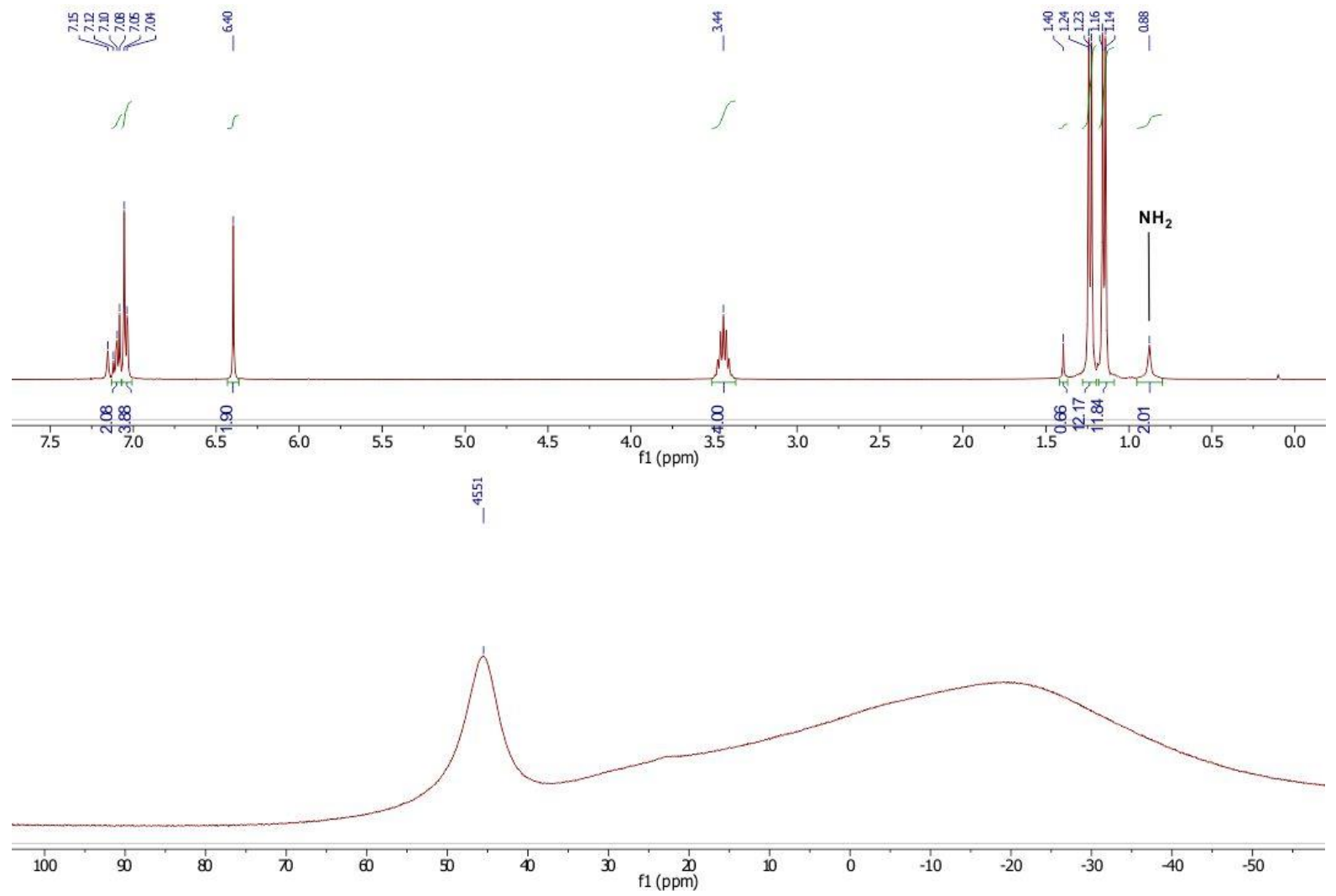

Figure s13: ${ }^{1} \mathrm{H}$ and ${ }^{11} \mathrm{~B}$ NMR spectra of crystalline $\left\{\mathrm{Sn}\left\{\mathrm{B}(\mathrm{NDippCH})_{2}\right\}\left(\mu-\mathrm{NH}_{2}\right)\right\}_{2}$ (the peak at $\delta_{\mathrm{H}} 1.40 \mathrm{ppm}$ belongs to residual cyclohexane) 


\section{Additional experiments on ammonia activation chemistry}

Reaction of $\operatorname{Sn}\left\{\mathrm{B}(\mathrm{NDippCH})_{2}\right\}_{2}$ (1) with excess ammonia. A degassed solution of $\mathrm{Sn}\left\{\mathrm{B}(\mathrm{NArCH})_{2}\right\}_{2}(\mathbf{1})(25 \mathrm{mg}, 0.028 \mathrm{mmol})$ in $\mathrm{C}_{6} \mathrm{D}_{6}(0.5 \mathrm{~mL})$ was briefly exposed to an atmosphere of dry ammonia, the mixture was shaken and monitored by ${ }^{1} \mathrm{H} \mathrm{NMR}$ for $24 \mathrm{~h}$. Initial spectrum showed that the ratio of 1 to $\mathrm{NH}_{3}$ was $\sim 1: 1.9$. The signals of the initially formed $\mathbf{1} \mathrm{NH}_{3}$ disappeared after $21 \mathrm{~h}$.

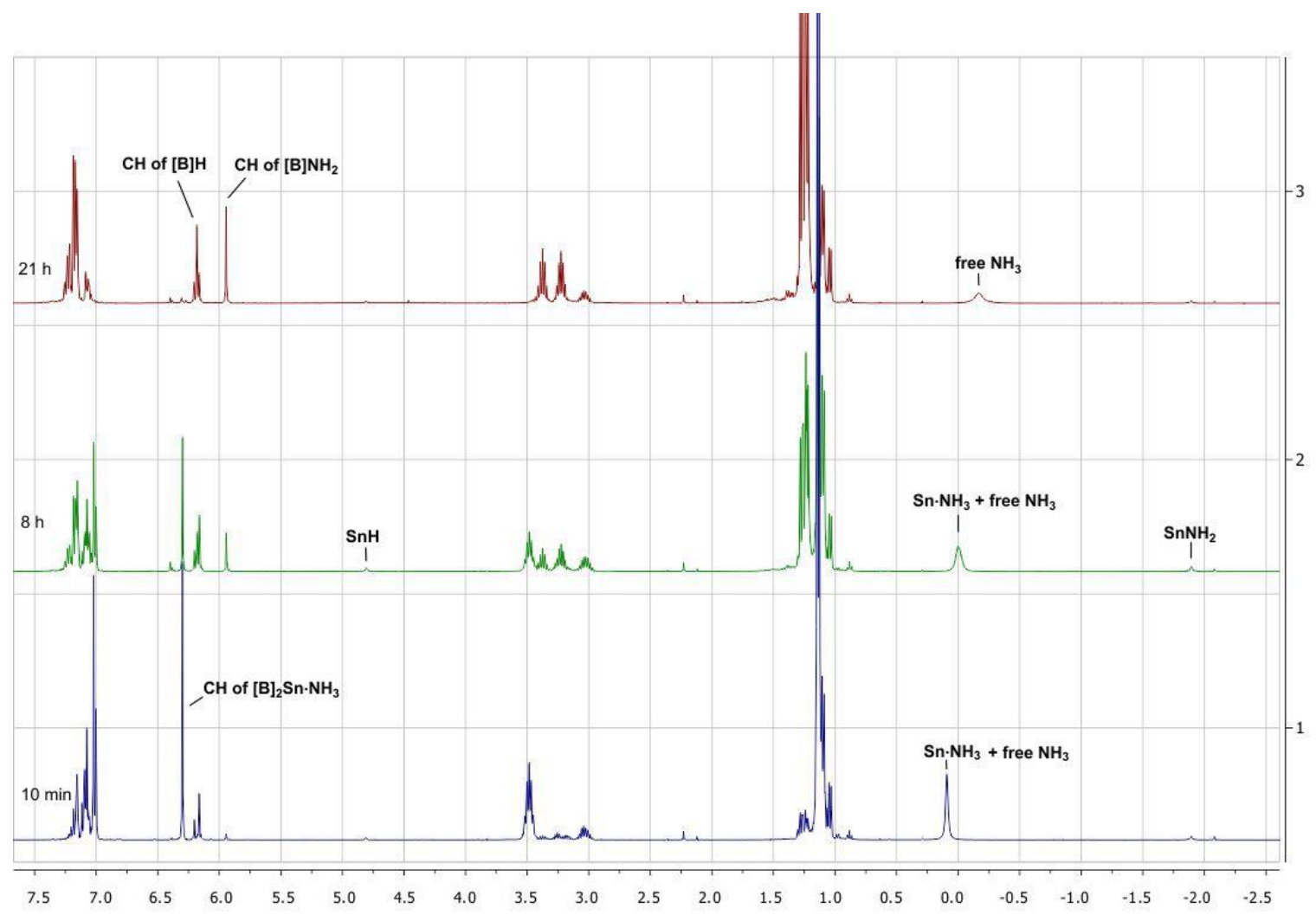

Figure s14: ${ }^{1} \mathrm{H}$ NMR $\left(\mathrm{C}_{6} \mathrm{D}_{6}\right)$ spectra of the reaction mixture $\mathbf{1}+\mathrm{NH}_{3}$ (excess)

Reaction of $\mathrm{Sn}\left\{\mathrm{B}(\mathrm{NDippCH})_{2}\right\}_{2}\left(\mathrm{NH}_{3}\right)\left(1 \mathrm{NH}_{3}\right)$ with 0.15 equiv. of $\mathrm{KN}\left(\mathrm{SiMe}_{3}\right)_{2}$. A solution of $\mathrm{Sn}\left\{\mathrm{B}(\mathrm{NArCH})_{2}\right\}_{2}\left(\mathrm{NH}_{3}\right)\left(\mathbf{1} \mathrm{NH}_{3}\right)(21 \mathrm{mg}, 0.024 \mathrm{mmol})$ in $\mathrm{C}_{6} \mathrm{D}_{6}(0.5 \mathrm{~mL})$ was first checked by ${ }^{1} \mathrm{H} \mathrm{NMR}$, then $\mathrm{KN}\left(\mathrm{SiMe}_{3}\right)_{2}$ (approx. $0.5 \mathrm{mg}, 0.0025 \mathrm{mmol}$ ) was added; the mixture was shaken and monitored by ${ }^{1} \mathrm{H}$ NMR for several days. Integration of the $\mathbf{1} \mathrm{NH}_{3}$ and $\mathrm{HN}\left(\mathrm{SiMe}_{3}\right)_{2}$ signals in the initial spectrum showed that the ratio of $\mathbf{1} \mathrm{NH}_{3}$ to 
$\mathrm{KN}\left(\mathrm{SiMe}_{3}\right)_{2}$ was $\sim 1: 0.15$. Immediately after $\mathrm{KN}\left(\mathrm{SiMe}_{3}\right)_{2}$ addition, compound 9 was formed, which slowly decomposed to $1: 1$ mixture of $\mathrm{H}_{2} \mathrm{NB}(\mathrm{NDippCH})_{2}$ and $\mathrm{HB}(\mathrm{NDippCH})_{2}$ and some unidentified compounds. Practically no changes in the spectrum were observed after $30 \mathrm{~h}$.

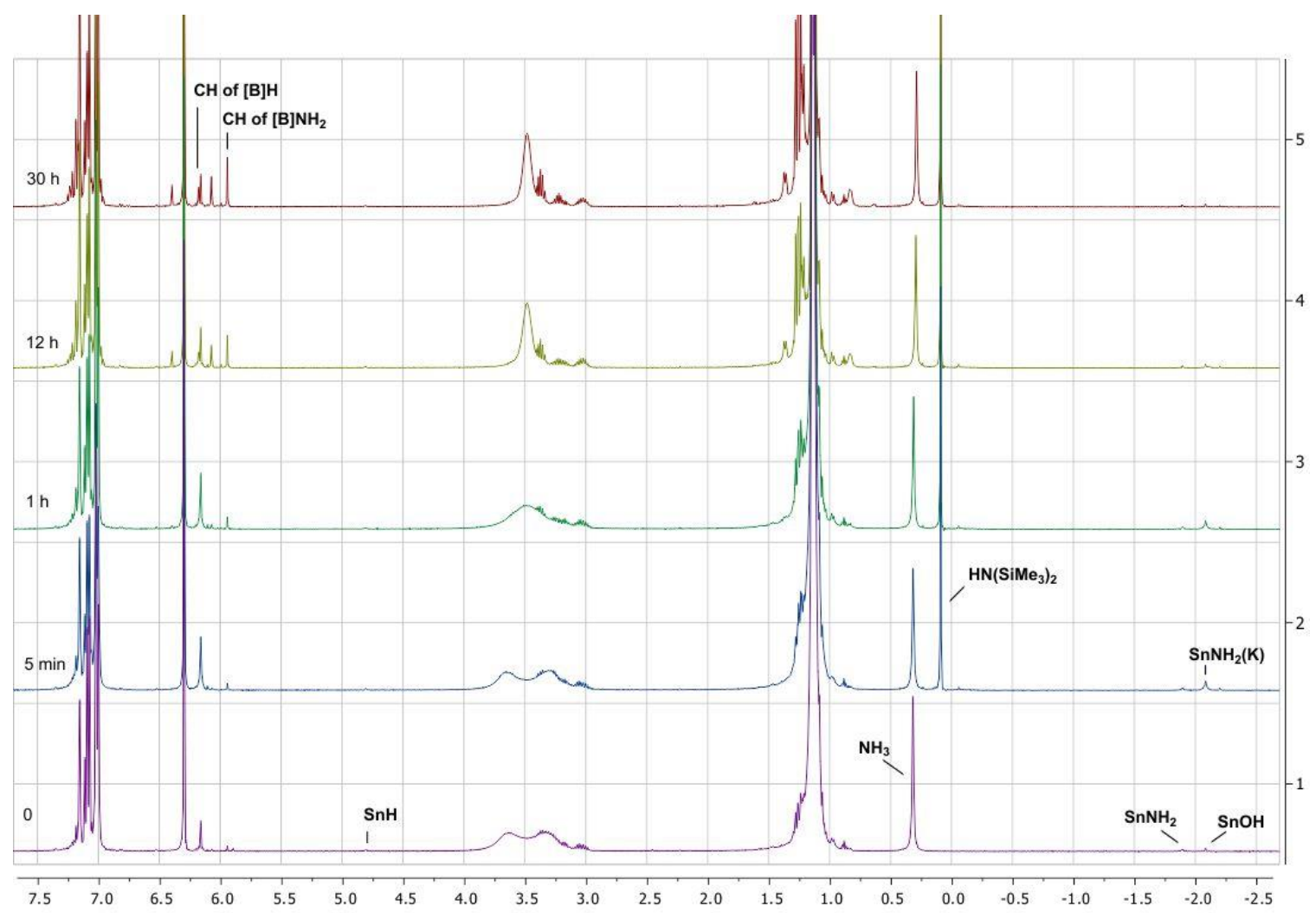

Figure s15: ${ }^{1} \mathrm{H}$ NMR $\left(\mathrm{C}_{6} \mathrm{D}_{6}\right)$ spectra of the reaction mixture $\mathbf{1} \mathbf{N H}_{3}+\mathrm{KN}\left(\mathrm{SiMe}_{3}\right)_{2}(0.15$ equiv.)

Reaction of $\mathrm{Sn}\left\{\mathrm{B}(\mathrm{NDippCH})_{2}\right\}_{2}\left(\mathrm{NH}_{3}\right) \quad\left(1 \mathrm{NH}_{3}\right) \quad$ with $\quad 0.06$ equiv. of $\left[\mathbf{P h N M e}_{2} \mathbf{H}\right]\left[\mathbf{B}\left(\mathbf{C}_{6} \mathbf{F}_{5}\right)_{4}\right]$. A solution of $\mathrm{Sn}\left\{\mathrm{B}(\mathrm{NArCH})_{2}\right\}_{2}\left(\mathrm{NH}_{3}\right)\left(\mathbf{1} \mathrm{NH}_{3}\right)(24 \mathrm{mg}, 0.027$ mmol) in $\mathrm{C}_{6} \mathrm{D}_{6}(0.5 \mathrm{~mL})$ was first checked by ${ }^{1} \mathrm{H}$ NMR, then $\left[\mathrm{PhNMe}_{2} \mathrm{H}\right]\left[\mathrm{B}\left(\mathrm{C}_{6} \mathrm{~F}_{5}\right)_{4}\right](1.3$ $\mathrm{mg}, 0.0016 \mathrm{mmol}$ ) was added; the mixture was shaken and monitored by ${ }^{1} \mathrm{H}$ NMR for one day. Acid-catalysed conversion of $\mathbf{1} \mathrm{NH}_{3}$ to $\mathbf{7}$ was essentially complete after $3 \mathrm{~h}$, then progressive decomposition of 7 to 1:1 mixture of $\mathrm{H}_{2} \mathrm{NB}(\mathrm{NDippCH})_{2}$ and $\mathrm{HB}(\mathrm{NDippCH})_{2}$ was observed. 


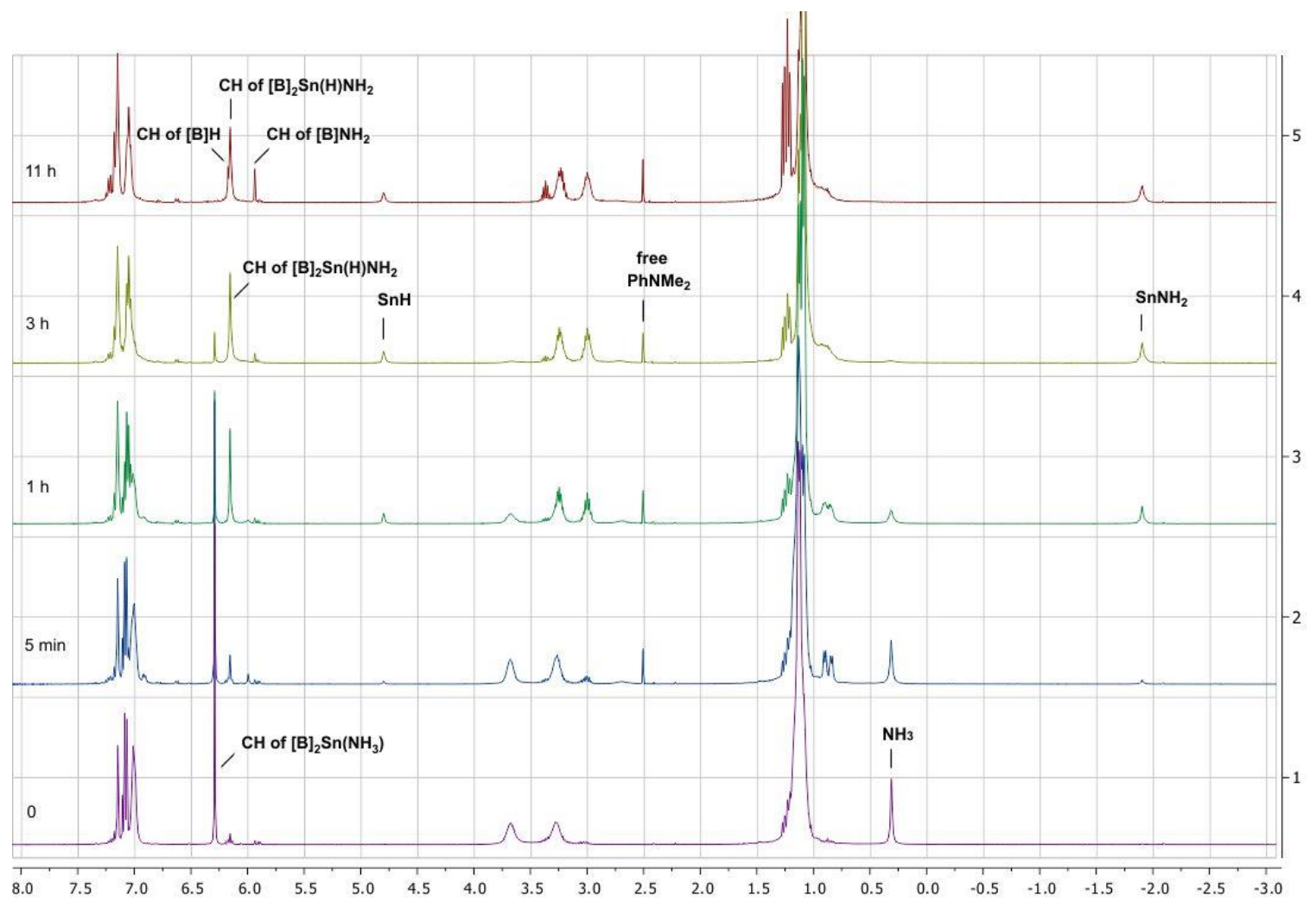

Figure s16: ${ }^{1} \mathrm{H} \quad \mathrm{NMR} \quad\left(\mathrm{C}_{6} \mathrm{D}_{6}\right)$ spectra of the reaction mixture $\mathbf{1} \mathrm{NH}_{3}+$ $\left[\mathrm{PhNMe}{ }_{2} \mathrm{H}\right]\left[\mathrm{B}\left(\mathrm{C}_{6} \mathrm{~F}_{5}\right)_{4}\right]$ (0.06 equiv.) 


\section{Additional synthetic data}

Synthesis of $\mathbf{H}_{2} \mathbf{N B}(\mathbf{N D i p p C H})_{2}$. A degassed suspension of $\mathrm{KCH}_{2} \mathrm{Ph}(10 \mathrm{mg}, 0.077$ $\mathrm{mmol})$ in $\mathrm{C}_{6} \mathrm{H}_{6}(2 \mathrm{~mL})$ was exposed to an atmosphere of ammonia and shaken by hand until the orange color disappeared, indicating the formation of white $\mathrm{KNH}_{2}$. Solid $\mathrm{BrB}(\mathrm{NDippCH})_{2}(32 \mathrm{mg}, 0.068 \mathrm{mmol})$ was added to the resulting suspension producing copious precipitate. After stirring for $1 \mathrm{~h}$ at $20^{\circ} \mathrm{C}$ all volatiles were removed in vacuo, the residue was extracted with hexane $(1 \mathrm{~mL})$, and the extract slowly evaporated at $20{ }^{\circ} \mathrm{C}$, while colorless crystals were growing, which were washed with a small amount of cold hexane and dried in vacuo yielding $\mathrm{H}_{2} \mathrm{NB}(\mathrm{NDippCH})_{2}(23 \mathrm{mg}, 0.057 \mathrm{mmol}(\mathrm{MW}=$ 403.42), 84\%).

${ }^{1} \mathrm{H}$ NMR $\left(\mathrm{C}_{6} \mathrm{D}_{6}\right): \delta 7.23(2 \mathrm{H}, \mathrm{m}, p-\mathrm{H}$ of $\mathrm{Ar}), 7.18\left(4 \mathrm{H}, \mathrm{d},{ }^{3} J=7.7 \mathrm{~Hz}, m-\mathrm{H}\right.$ of Ar), 5.94

$(2 \mathrm{H}, \mathrm{s}, \mathrm{NCH}), 3.37\left(4 \mathrm{H}\right.$, septet, $\left.{ }^{3} J=6.9 \mathrm{~Hz}, \mathrm{CHMe}\right), 1.27\left(12 \mathrm{H}, \mathrm{d},{ }^{3} J=6.9 \mathrm{~Hz}\right.$, $\left.\mathrm{CHMe} e_{2}\right), 1.25\left(12 \mathrm{H}, \mathrm{d},{ }^{3} \mathrm{~J}=6.9 \mathrm{~Hz}, \mathrm{CHMe} 2\right), 1.19\left(2 \mathrm{H}\right.$, br s, $\left.\mathrm{NH}_{2}\right)$.

${ }^{13} \mathrm{C}\left\{{ }^{1} \mathrm{H}\right\} \mathrm{NMR}\left(\mathrm{C}_{6} \mathrm{D}_{6}\right): \delta 147.49$ (o-C of Ar), 138.67 (ipso-C of Ar), 127.51 ( $p-\mathrm{CH}$ of $\mathrm{Ar}$ ), $123.79(m-\mathrm{CH}$ of $\left.\mathrm{Ar}), 116.85(\mathrm{NCH}), 28.54\left(\mathrm{CHMe}_{2}\right), 24.33(\mathrm{CHMe})_{2}\right), 24.24\left(\mathrm{CHMe}_{2}\right)$.

${ }^{11} \mathrm{~B}\left\{{ }^{1} \mathrm{H}\right\}\left(\mathrm{C}_{6} \mathrm{D}_{6}\right): \delta 23.0$.

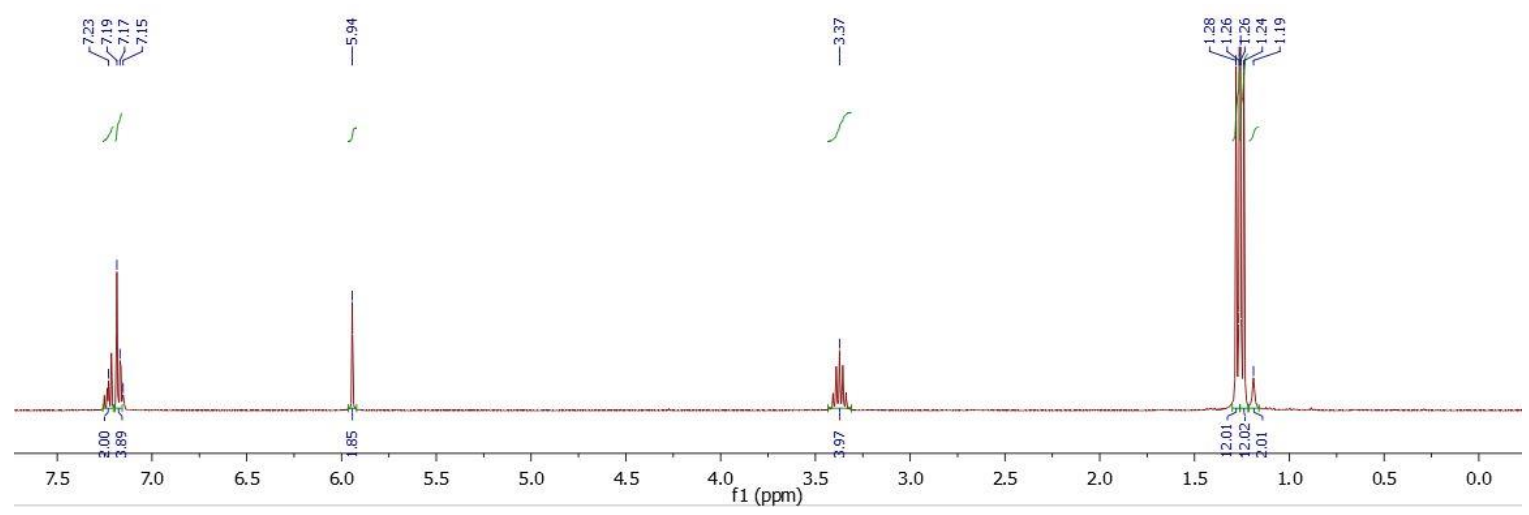




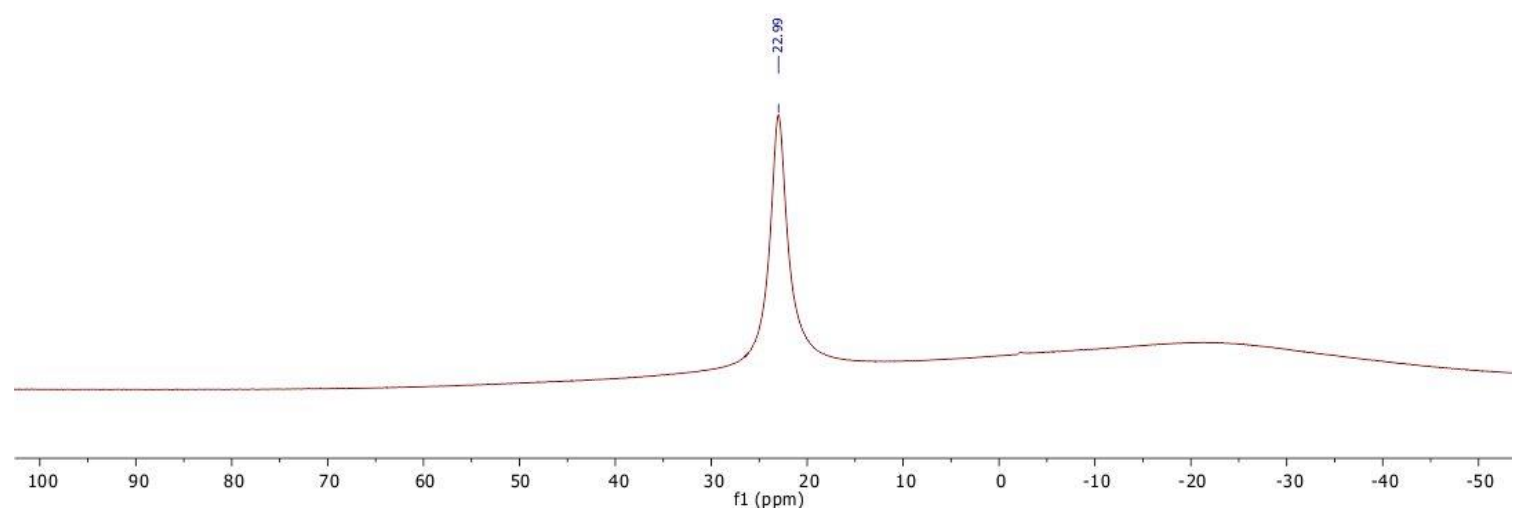

Figure s17: ${ }^{1} \mathrm{H}$ and ${ }^{11} \mathrm{~B}$ NMR spectra of crystalline $\mathrm{H}_{2} \mathrm{NB}(\mathrm{NDippCH})_{2}$

\section{Synthesis of $\mathrm{Si}\left\{\mathrm{NDipp}\left(\mathrm{SiMe}_{3}\right)\right\}\left\{\mathrm{Si}\left(\mathrm{SiMe}_{3}\right)_{3}\right\}(\mathrm{H})\left(\mathrm{NH}_{2}\right)$}

A degassed solution of $\operatorname{Si}\left\{\mathrm{NDipp}\left(\mathrm{SiMe}_{3}\right)\right\}\left\{\mathrm{Si}\left(\mathrm{SiMe}_{3}\right)_{3}\right\}(24 \mathrm{mg}, 0.046 \mathrm{mmol})$ in $\mathrm{C}_{6} \mathrm{H}_{6}$ $(0.5 \mathrm{~mL})$ was exposed to the atmosphere of dry ammonia gas. Upon shaking the mixture changed from bright purple to pale yellow. All volatiles removed in vacuo, the residue was dissolved in a minimal amount of hexane $(\sim 0.2 \mathrm{~mL})$ and the tube was sealed under vacuum. After crystallisation was initiated by freezing with liquid $\mathrm{N}_{2}$, the solution was stored at $-30{ }^{\circ} \mathrm{C}$ while large colourless blocks formed. The resulting crystals were washed with a small amount of cold hexane and dried in vacuo yielding $\mathrm{Si}\left\{\mathrm{NDipp}\left(\mathrm{SiMe}_{3}\right)\right\}\left\{\mathrm{Si}\left(\mathrm{SiMe}_{3}\right)_{3}\right\}(\mathrm{H})\left(\mathrm{NH}_{2}\right)(17 \mathrm{mg}, \mathrm{MW}=541.24,0.031 \mathrm{mmol}, 68.3 \%)$. Anal. found (calcd. for $\mathrm{C}_{24} \mathrm{H}_{56} \mathrm{~N}_{2} \mathrm{Si}_{6}$ ): C, 53.29 (53.26); H, 10.50 (10.43); N, 5.06 (5.18) $\%$.

${ }^{1} \mathrm{H}$ NMR $\left(\mathrm{C}_{6} \mathrm{D}_{6}\right): \delta 7.07(3 \mathrm{H}, \mathrm{m}, m$ - and $p-\mathrm{H}$ of $\mathrm{Ar}), 5.22\left(1 \mathrm{H}, \mathrm{t},{ }^{3} J=2.95 \mathrm{~Hz}\right.$, with ${ }^{29} \mathrm{Si}$ satellites $\left.{ }^{1} J(\mathrm{Si}-\mathrm{H})=214.4 \mathrm{~Hz}, \mathrm{SiH}\right), 3.65\left(1 \mathrm{H}\right.$, septet, $\left.{ }^{3} J=6.85 \mathrm{~Hz}, \mathrm{CHMe}\right), 3.56(1 \mathrm{H}$, septet, $\left.{ }^{3} J=6.81 \mathrm{~Hz}, \mathrm{CHMe}\right), 1.26\left(3 \mathrm{H}, \mathrm{d},{ }^{3} J=6.85 \mathrm{~Hz}, \mathrm{CHMe}\right), 1.25\left(3 \mathrm{H}, \mathrm{d},{ }^{3} J=6.85\right.$ $\mathrm{Hz}, \mathrm{CHMe}$ ), $1.24\left(3 \mathrm{H}, \mathrm{d},{ }^{3} J=6.85 \mathrm{~Hz}, \mathrm{CH} M e_{2}\right), 1.13\left(3 \mathrm{H}, \mathrm{d},{ }^{3} \mathrm{~J}=6.85 \mathrm{~Hz}, \mathrm{CH} M e_{2}\right)$, 0.99 (2 H, br s, $\left.\mathrm{NH}_{2}\right), 0.38$ (27 H, s, $\left.\mathrm{SiSiMe}_{3}\right), 0.23$ (9 H, s, NSiMe 3 ).

${ }^{13} \mathrm{C}\left\{{ }^{1} \mathrm{H}\right\} \operatorname{NMR}\left(\mathrm{C}_{6} \mathrm{D}_{6}\right): \delta 147.06$ (o-C of Ar), 146.73 (o-C of Ar), 144.54 (ipso-C of Ar), 125.28 ( $p$-CH of Ar), 124.39 ( $m$-CH of Ar), 124.01 ( $m-\mathrm{CH}$ of Ar), $28.08\left(C \mathrm{HMe}_{2}\right), 28.01$ $\left(\mathrm{CHMe}_{2}\right), 26.36(\mathrm{CHMe}), 26.31(\mathrm{CHMe}), 24.56(\mathrm{CHMe}), 24.20\left(\mathrm{CHMe} e_{2}\right), 3.60$ $\left(\mathrm{SiSiMe}_{3}\right), 3.26\left(\mathrm{NSiMe}_{3}\right)$. 

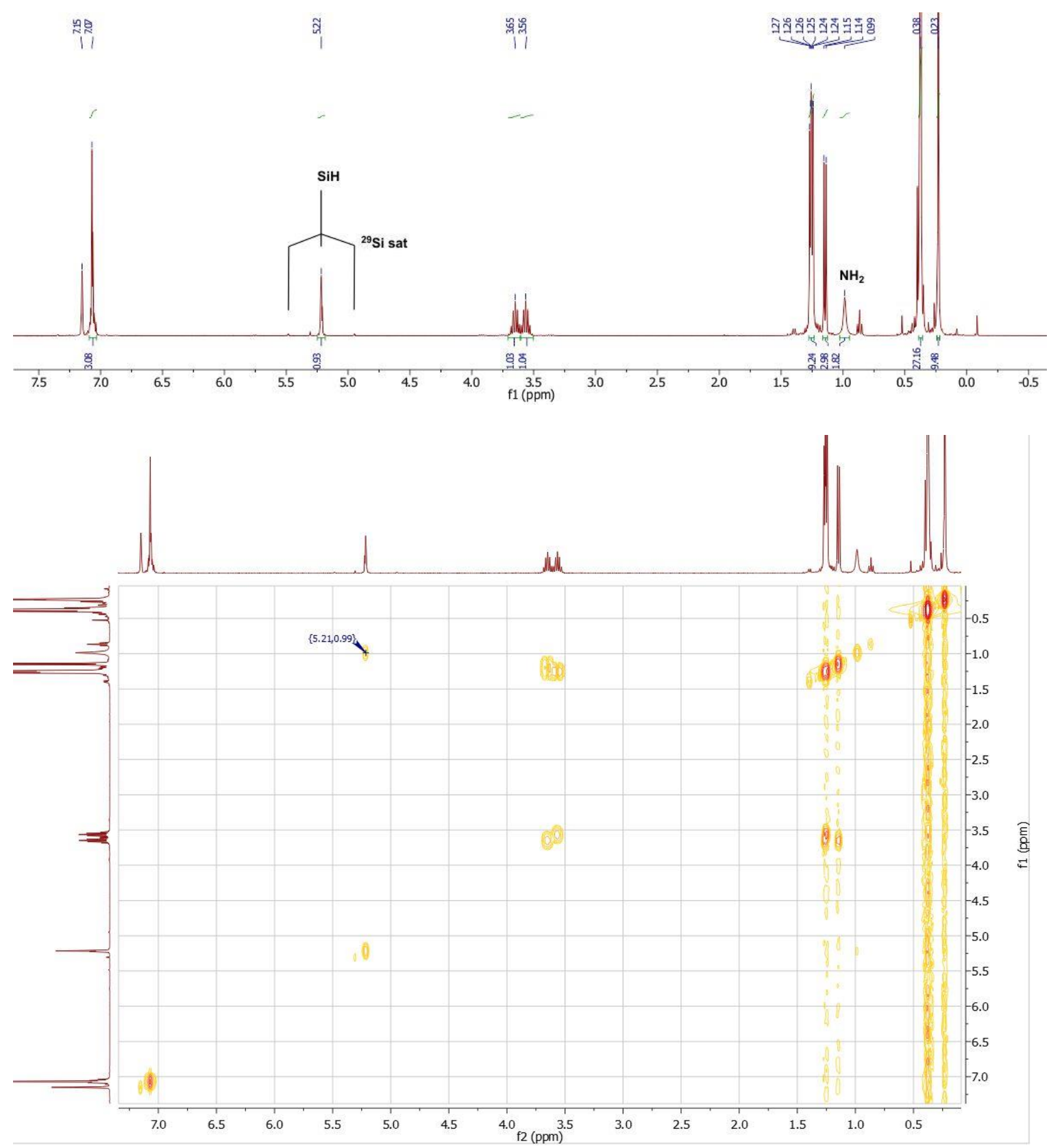

Figure s18: ${ }^{1} \mathrm{H}$ and ${ }^{1} \mathrm{H} /{ }^{1} \mathrm{H} \quad \mathrm{COSY} \quad \mathrm{NMR} \quad$ spectra of crystalline $\operatorname{Si}\left\{\mathrm{NDipp}\left(\mathrm{SiMe}_{3}\right)\right\}\left\{\mathrm{Si}\left(\mathrm{SiMe}_{3}\right)_{3}\right\}(\mathrm{H})\left(\mathrm{NH}_{2}\right)$ 
Synthesis of $\mathbf{C l}_{2} \mathbf{S n}\left\{\mathbf{B}(\mathbf{N D i p p C H})_{2}\right\}_{2}$. Method $A$. To a solution of $\mathrm{UCl}_{4}(20 \mathrm{mg}, 52.6$ $\mu \mathrm{mol})$ in THF-d $\mathrm{d}_{8}(0.25 \mathrm{~mL})$ was added a solution of $\mathrm{Sn}\left\{\mathrm{B}(\mathrm{NDippCH})_{2}\right\}_{2}(\mathbf{1 a})(47 \mathrm{mg}$, $52.6 \mu \mathrm{mol})$ in THF- $\mathrm{d}_{8}(0.25 \mathrm{~mL})$, producing a pale green solution that was allowed to react overnight. All the volatiles were removed under reduced pressure leaving a yellowgreen residue which was extracted into hexane and filtered. Slow evaporation of the hexane solution produced light yellow crystals, which were determined by an X-ray diffraction study to be $\mathrm{Cl}_{2} \mathrm{Sn}\left\{\mathrm{B}(\mathrm{NDippCH})_{2}\right\}_{2}$.

Method B. Trityl chloride $(13.7 \mathrm{mg}, 0.050 \mathrm{mmol})$ was added to a solution of $\mathrm{Sn}\left\{\mathrm{B}(\mathrm{NDippCH})_{2}\right\}_{2}$ (1a) $(22 \mathrm{mg}, 0.025 \mathrm{mmol})$ in $\mathrm{C}_{6} \mathrm{D}_{6}(0.5 \mathrm{~mL})$. Almost immediately the yellow-green color changed to yellow and ${ }^{1} \mathrm{H}$ NMR spectrum showed clean formation of two new species (the known trityl dimer and the tin boryl compound as in Method A). The mixture was transferred into a two-section crystallization tube and all volatiles removed in vacuo. Hexane $(1 \mathrm{~mL})$ was added to the residue and the tube was sealed under vacuum. During crystallisation a partial decomposition occurred (the solution turned light brown) yielding slightly impure product (15 mg, MW = 964.41, $0.016 \mathrm{mmol}$, $62.2 \%)$.

${ }^{1} \mathrm{H}$ NMR $\left(\mathrm{C}_{6} \mathrm{D}_{6}\right): \delta 7.17\left(4 \mathrm{H}, \mathrm{t},{ }^{3} J=7.7 \mathrm{~Hz}, p-\mathrm{H}\right.$ of $\left.\mathrm{Ar}\right), 7.07\left(8 \mathrm{H}, \mathrm{d},{ }^{3} J=7.7 \mathrm{~Hz}, m-\mathrm{H}\right.$ of Ar), $6.06\left(4 \mathrm{H}\right.$, s, with ${ }^{119 / 117} \mathrm{Sn}$ satellites $\left.{ }^{4} J(\mathrm{Sn}-\mathrm{H})=14.2 \mathrm{~Hz}, \mathrm{NCH}\right), 3.07$ (8 H, septet, ${ }^{3} J$ $=6.8 \mathrm{~Hz}, \mathrm{CHMe} 2), 1.17\left(24 \mathrm{H}, \mathrm{d},{ }^{3} J=6.8 \mathrm{~Hz}, \mathrm{CHMe}\right), 1.09\left(24 \mathrm{H}, \mathrm{d},{ }^{3} J=6.8 \mathrm{~Hz}\right.$, $\mathrm{CHMe}$ ).

${ }^{13} \mathrm{C}\left\{{ }^{1} \mathrm{H}\right\}$ NMR $\left(\mathrm{C}_{6} \mathrm{D}_{6}\right): \delta 145.92$ (o-C of Ar), 138.97 (ipso-C of Ar), 128.39 ( $p-\mathrm{CH}$ of Ar), $123.91\left(m-\mathrm{CH}\right.$ of $\mathrm{Ar}$ ), 123.89 (s with ${ }^{119 / 117} \mathrm{Sn}$ satellites $\left.{ }^{3} J(\mathrm{Sn}-\mathrm{H})=49 \mathrm{~Hz}, \mathrm{NCH}\right), 28.85$ $\left(\mathrm{CHMe}_{2}\right), 26.01(\mathrm{CHMe}), 23.85\left(\mathrm{CHMe} e_{2}\right)$.

${ }^{11} \mathrm{~B}\left\{{ }^{1} \mathrm{H}\right\}\left(\mathrm{C}_{6} \mathrm{D}_{6}\right): \delta 27.8\left(\mathrm{br}, \Delta w_{1 / 2}=306 \mathrm{~Hz}\right.$ with ${ }^{119 / 117} \mathrm{Sn}$ satellites $\left.{ }^{1} J(\mathrm{Sn}-\mathrm{B})=1150 \mathrm{~Hz}\right)$. 


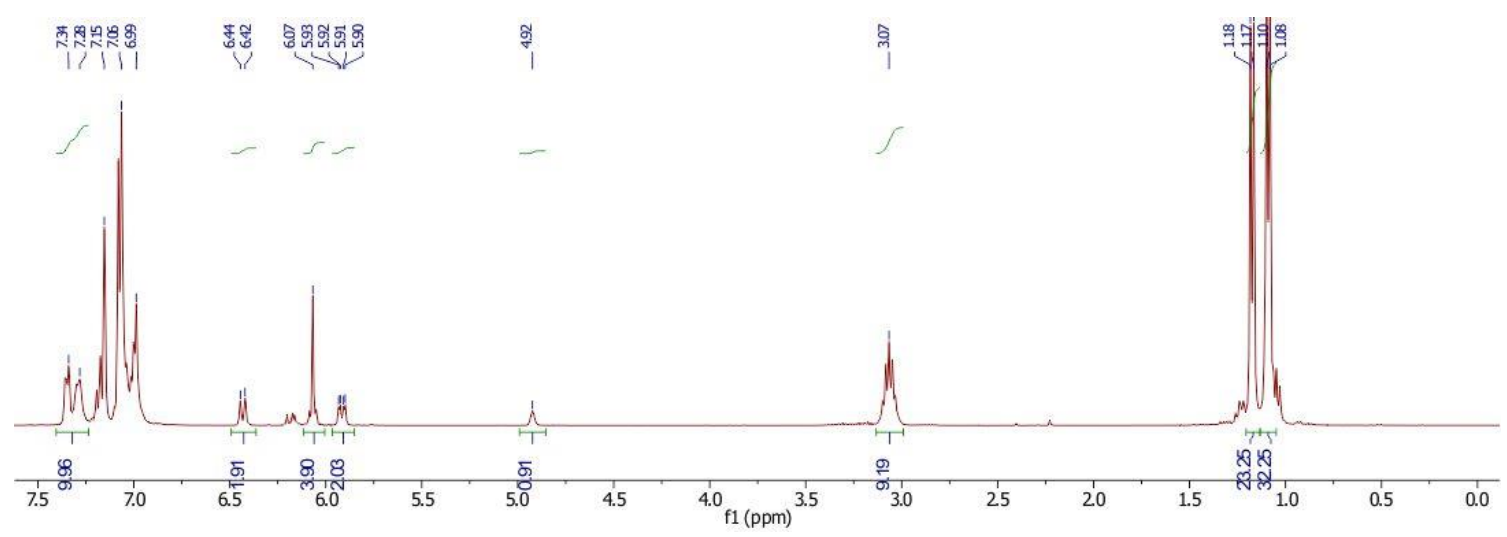

Figure s19: ${ }^{1} \mathrm{H}$ NMR spectrum of the reaction mixture $\mathrm{Sn}\left\{\mathrm{B}(\mathrm{NDippCH})_{2}\right\}_{2}+2 \mathrm{Ph}_{3} \mathrm{CCl}$ showing almost quantitative formation of $\mathrm{Cl}_{2} \mathrm{Sn}\left\{\mathrm{B}(\mathrm{NDippCH})_{2}\right\}_{2}$ and trityl dimer

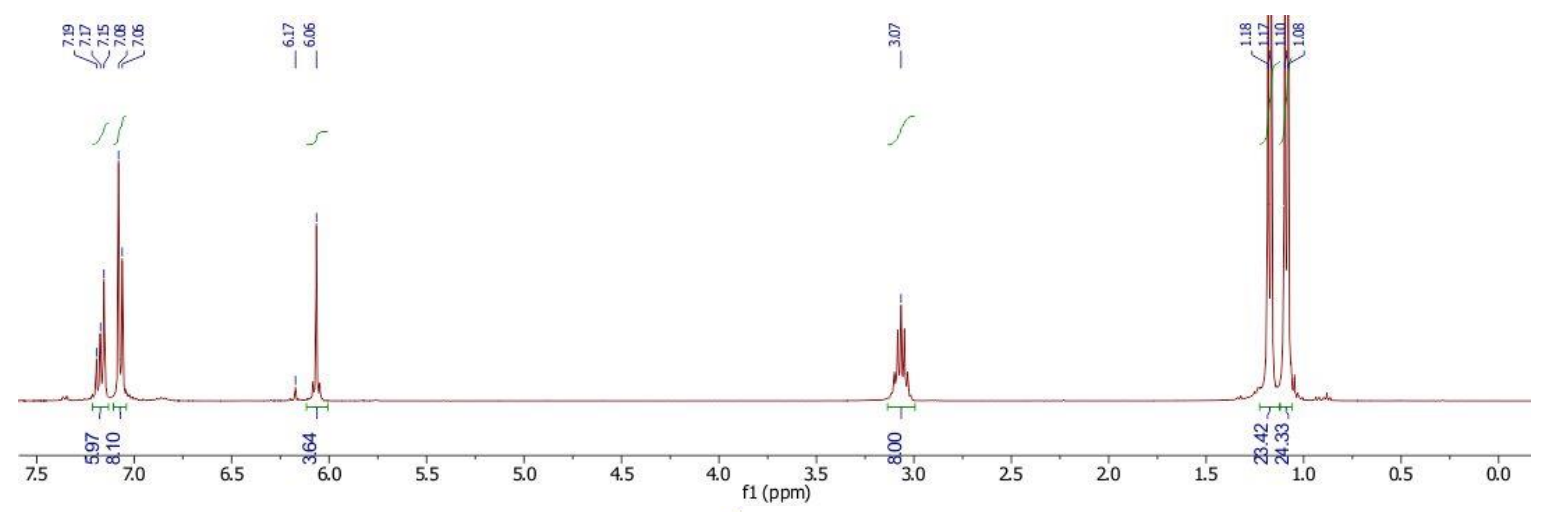

量

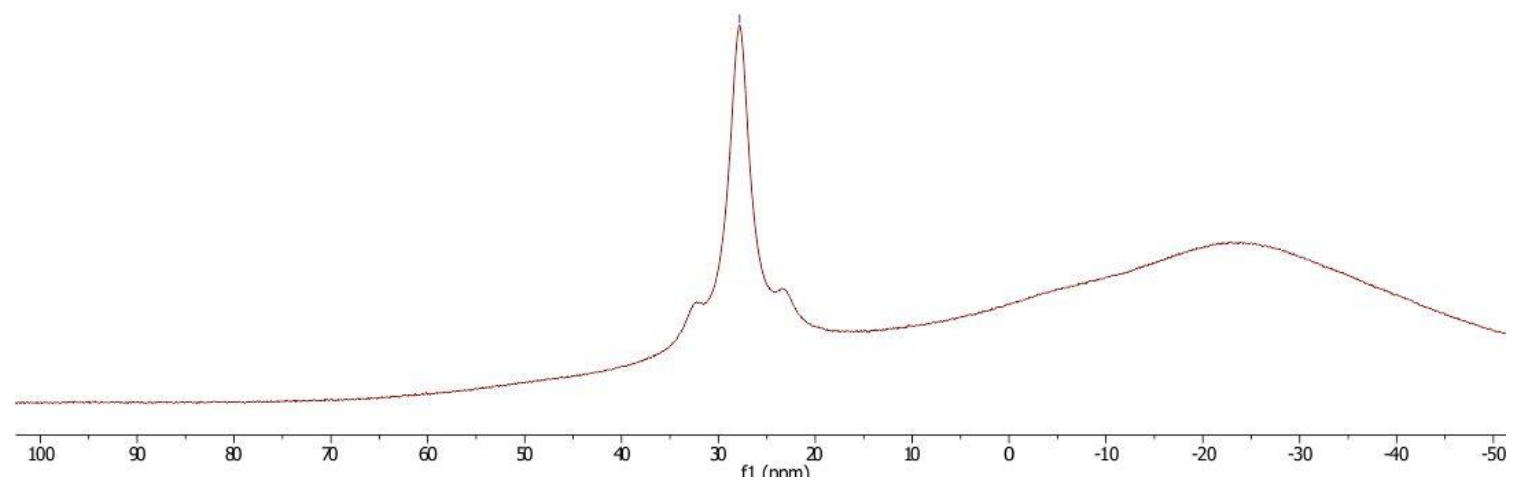

Figure s20: ${ }^{1} \mathrm{H}$ and ${ }^{11} \mathrm{~B}\left\{{ }^{1} \mathrm{H}\right\}$ NMR spectra of crystalline $\mathrm{Cl}_{2} \mathrm{Sn}\left\{\mathrm{B}(\mathrm{NDippCH})_{2}\right\}_{2}$

\section{Synthesis of $\left\{\left(\mathrm{MeCCH}_{2}\right)_{2}\right\} \operatorname{Sn}\left\{\mathrm{NDipp}\left(\mathrm{SiMe}_{3}\right)\right\}\left\{\mathrm{B}(\mathrm{NDippCH})_{2}\right\}$}

To a solution of $\mathrm{Sn}\left\{\mathrm{NDipp}\left(\mathrm{SiMe}_{3}\right)\right\}\left\{\mathrm{B}(\mathrm{NDippCH})_{2}\right\}$ (1b) $(38 \mathrm{mg}, 0.050 \mathrm{mmol})$ in $\mathrm{C}_{6} \mathrm{D}_{6}$ $(0.5 \mathrm{~mL})$ was added excess 2,3-dimethylbutadiene $(30 \mu \mathrm{L}, 22 \mathrm{mg}, 0.265 \mathrm{mmol}) .{ }^{1} \mathrm{H}$ NMR 
spectrum showed that only a small amount of product was formed. Upon storing at $20^{\circ} \mathrm{C}$ for $2 \mathrm{~h}$ the mixture changed from bright purple to almost colourless and the signals of $\mathbf{1 b}$ disappeared. The mixture was transferred into a small crystallisation tube, all volatiles removed in vacuo, the residue was dissolved in a minimal amount of pentane $(\sim 0.2 \mathrm{~mL})$ and the tube was sealed under vacuum. After crystallisation was initiated by freezing with liquid $\mathrm{N}_{2}$, the solution was stored at $4{ }^{\circ} \mathrm{C}$ while large colourless blocks formed. The resulting crystals were washed with a small amount of cold pentane and dried in vacuo yielding $\left\{\left(\mathrm{MeCCH}_{2}\right)_{2}\right\} \mathrm{Sn}\left\{\mathrm{NDipp}\left(\mathrm{SiMe}_{3}\right)\right\}\left\{\mathrm{B}(\mathrm{NDippCH})_{2}\right\}(22 \mathrm{mg}, \mathrm{MW}=836.72,0.026$ mmol, $52.6 \%$ ).

${ }^{1} \mathrm{H}$ NMR $\left(\mathrm{C}_{6} \mathrm{D}_{6}\right): \delta 7.23\left(2 \mathrm{H}, \mathrm{t},{ }^{3} J=7.7 \mathrm{~Hz}, p-\mathrm{H}\right.$ of Ar/boryl $), 7.13\left(4 \mathrm{H}, \mathrm{d},{ }^{3} J=7.7 \mathrm{~Hz}\right.$, $m-\mathrm{H}$ of Ar /boryl $\left.+\mathrm{C}_{6} \mathrm{D}_{5} \mathrm{H}\right), 7.00(3 \mathrm{H}, \mathrm{m}, m$ - and $p-\mathrm{H}$ of Ar/amide), $6.25(2 \mathrm{H}$, s, with ${ }^{119 / 117} \mathrm{Sn}$ satellites $\left.{ }^{4} J(\mathrm{Sn}-\mathrm{H})=11.9 \mathrm{~Hz}, \mathrm{NCH}\right), 3.36\left(2 \mathrm{H}\right.$, septet, $\left.{ }^{3} J=6.8 \mathrm{~Hz}, \mathrm{CHMe} 2\right)$, $3.23\left(4 \mathrm{H}\right.$, septet, $\left.{ }^{3} J=6.8 \mathrm{~Hz}, \mathrm{CHMe}\right), 1.49\left(6 \mathrm{H}, \mathrm{s}, \mathrm{SnCH}_{2} \mathrm{CMe}\right), 1.35\left(2 \mathrm{H}, \mathrm{d},{ }^{2} J=15.8\right.$ $\left.\mathrm{Hz}, \mathrm{SnCH}_{a} \mathrm{H}_{\mathrm{b}} \mathrm{CMe}\right), 1.28\left(12 \mathrm{H}, \mathrm{d},{ }^{3} J=6.8 \mathrm{~Hz}, \mathrm{CHMe} e_{2}, 1.12\left(6 \mathrm{H}, \mathrm{d},{ }^{3} \mathrm{~J}=6.8 \mathrm{~Hz}\right.\right.$, $\mathrm{CHMe} 2), 1.10\left(12 \mathrm{H}, \mathrm{d},{ }^{3} J=6.8 \mathrm{~Hz}, \mathrm{CHMe}\right), 0.85\left(2 \mathrm{H}, \mathrm{d},{ }^{2} J=15.7 \mathrm{~Hz}, \mathrm{SnCH}_{\mathrm{a}} H_{b} \mathrm{CMe}\right)$, $0.75\left(6 \mathrm{H}, \mathrm{d},{ }^{3} \mathrm{~J}=6.8 \mathrm{~Hz}, \mathrm{CHMe} 2\right), 0.22\left(9 \mathrm{H}, \mathrm{s}, \mathrm{NSiMe}_{3}\right)$.

${ }^{13} \mathrm{C}\left\{{ }^{1} \mathrm{H}\right\}$ NMR $\left(\mathrm{C}_{6} \mathrm{D}_{6}\right): \delta 147.62$ (o-C of Ar/amide), 146.38 (o-C of Ar/boryl), 146.18 (ipso-C of Ar/amide), 140.37 (ipso-C of Ar/boryl), $131.90\left({ }^{119 / 117} \mathrm{Sn}\right.$ satellites ${ }^{2} J(\mathrm{Sn}-\mathrm{C})=$ $24.5 \mathrm{~Hz}, \mathrm{SnCH}_{2} \mathrm{CMe}$ ), 128.41 ( $p$-CH of Ar/boryl), 124.08 ( $m-\mathrm{CH}$ of Ar/boryl), 123.82 ( $m$-CH of Ar/amide), 123.62 ( $p$-CH of Ar/amide), $123.44\left({ }^{119 / 117} \mathrm{Sn}\right.$ satellites ${ }^{3} J(\mathrm{Sn}-\mathrm{C})=$ $7.6 \mathrm{~Hz}, \mathrm{NCH}$ ), 28.74 ( $\mathrm{CHMe}_{2} /$ boryl), 27.85 ( $\mathrm{CHMe}_{2} /$ amide), 26.69 (CHMe $/$ boryl), 25.68 $\left({ }^{119 / 117} \mathrm{Sn}\right.$ satellites overlapping, $\left.\mathrm{SnCH}_{2}\right), 25.64(\mathrm{CHMe} /$ amide $), 24.55\left(\mathrm{CHMe}_{2} / \mathrm{amide}\right)$, $23.19(\mathrm{CHMe} /$ boryl $), 21.35\left({ }^{119 / 117} \mathrm{Sn}\right.$ satellites $\left.{ }^{3} J(\mathrm{Sn}-\mathrm{C})=65.4 \mathrm{~Hz}, \mathrm{SnCH}_{2} \mathrm{CMe}\right), 3.38$ $\left(\mathrm{NSiMe}_{3}\right)$.

${ }^{11} \mathrm{~B}\left\{{ }^{1} \mathrm{H}\right\}\left(\mathrm{C}_{6} \mathrm{D}_{6}\right): \delta 29.5\left(\mathrm{br}, \Delta w_{1 / 2}=310 \mathrm{~Hz}\right.$ with ${ }^{119 / 117} \mathrm{Sn}$ satellites $\left.{ }^{1} J(\mathrm{Sn}-\mathrm{B})=1050 \mathrm{~Hz}\right)$. 

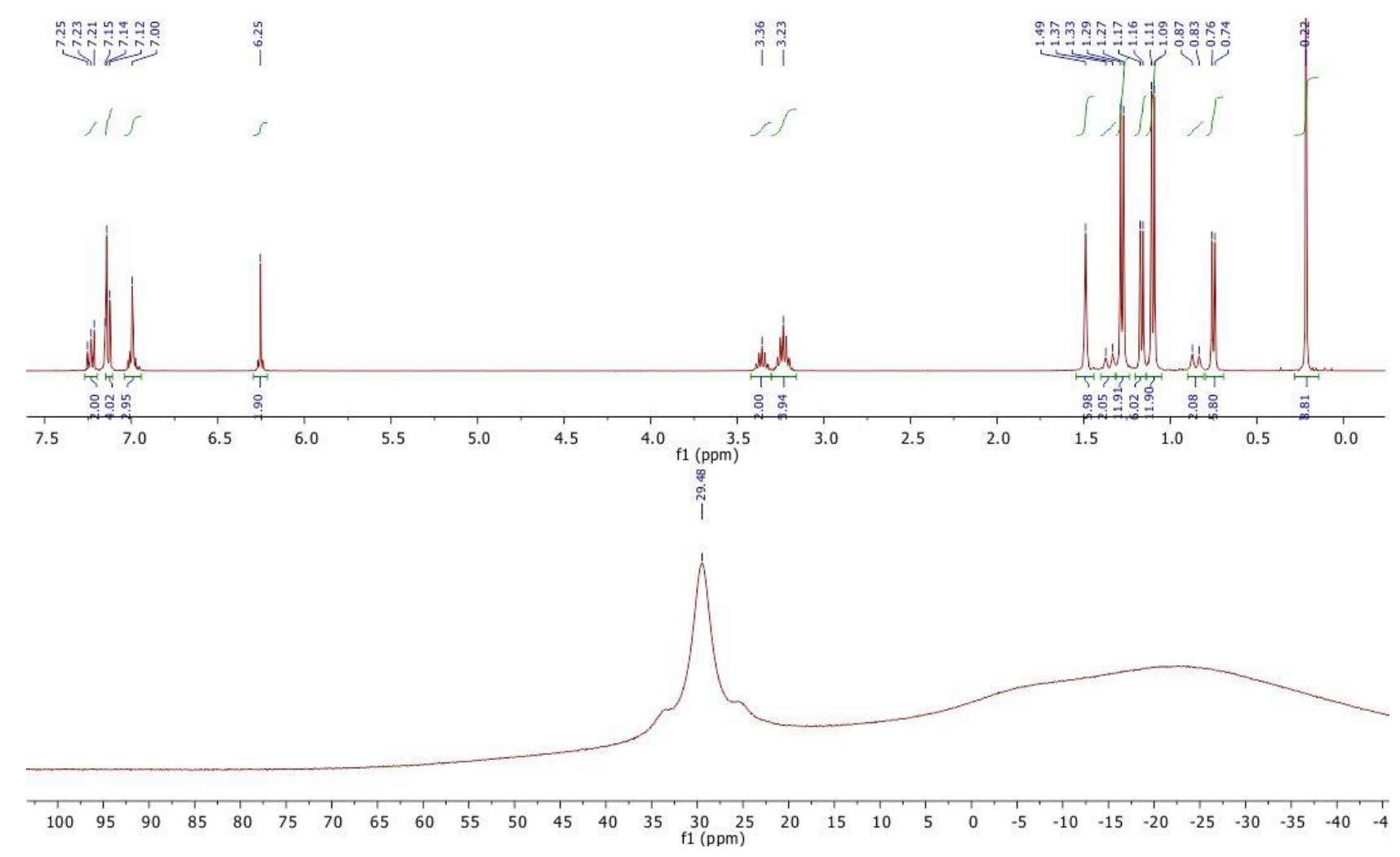

Figure s21: ${ }^{1} \mathrm{H}$ and ${ }^{11} \mathrm{~B}\left\{{ }^{1} \mathrm{H}\right\} \quad \mathrm{NMR} \quad$ spectra of the crystalline $\left\{\left(\mathrm{MeCCH}_{2}\right)_{2}\right\} \operatorname{Sn}\left\{\mathrm{NDipp}\left(\mathrm{SiMe}_{3}\right)\right\}\left\{\mathrm{B}(\mathrm{NDippCH})_{2}\right\}$

\section{Isolation of $\mathrm{K}\left[\mathrm{B}\left(\mathrm{C}_{6} \mathrm{~F}_{5}\right)_{4}\right]$ crystals}

Potassium amide $\mathrm{K}\left\{\mathrm{N}\left(\mathrm{SiMe}_{3}\right)_{2}\right\}(8.0 \mathrm{mg}, 0.040 \mathrm{mmol})$ was added to a suspension of $\left[\mathrm{Et}_{3} \mathrm{NH}\right]\left[\mathrm{B}\left(\mathrm{C}_{6} \mathrm{~F}_{5}\right)_{4}\right](31 \mathrm{mg}, 0.040 \mathrm{mmol})$ in $\mathrm{C}_{6} \mathrm{D}_{6}(0.5 \mathrm{~mL})$ in NMR tube. Initially drops of heavy liquid were formed, which turned into crystalline powder after sonication for 5 min. ${ }^{1} \mathrm{H}$ NMR showed that only triethylamine and $\mathrm{HN}\left(\mathrm{SiMe}_{3}\right)_{2}$ were present in solution. The liquid was carefully decanted from the precipitate using a long pipette and a solution of $\mathrm{Sn}\left\{\mathrm{B}(\mathrm{NArCH})_{2}\right\}_{2}\left(\mathrm{NH}_{3}\right)\left(\mathbf{1}^{\cdot} \mathrm{NH}_{3}\right)(34.5 \mathrm{mg}, 0.0379 \mathrm{mmol})$ in $\mathrm{C}_{6} \mathrm{D}_{6}(0.5 \mathrm{~mL})$ was added; after sonication the mixture for $10 \mathrm{~min}$ all the original precipitate of $\mathrm{K}\left[\mathrm{B}\left(\mathrm{C}_{6} \mathrm{~F}_{5}\right)_{4}\right]$ had dissolved and ${ }^{1} \mathrm{H}$ NMR showed the formation of $\mathrm{Sn}\left\{\mathrm{B}(\mathrm{NArCH})_{2}\right\}_{2}\left(\mathrm{NH}_{2}\right)(\mathrm{H})$ (7) as a major product. Attempted crystallisation of the latter by layering of the concentrated $\mathrm{C}_{6} \mathrm{D}_{6}$ solution with hexane resulted in polycrystalline material contaminated with brown tar (progressive darkening occurred during two days of solvent diffusion). Storing the supernatant solution at $20^{\circ} \mathrm{C}$ for two weeks resulted in precipitation of black powder 
(bottom) and formation of colourless plate-like crystals (on the walls). An X-Ray diffraction study revealed that the latter was unsolvated $\mathrm{K}\left[\mathrm{B}\left(\mathrm{C}_{6} \mathrm{~F}_{5}\right)_{4}\right]$. 


\section{Additional crystallographic data}

Crystal data for $n i d o-\mathrm{Sn}_{11}\left\{\mathrm{~B}(\mathrm{NDippCH})_{2}\right\}_{4} /$ nido-Sn ${ }_{10}\left\{\mathrm{~B}(\mathrm{NDippCH})_{2}\right\}_{4}$ co-crystallite: $a=$ 24.6958(2) $\AA, b=14.9348(1) \AA, c=33.1882(4) \AA, \beta=94.4855(9)^{\circ}$, monoclinic, $P 22_{1} / \mathrm{c}$, $V=12203.2(2) \AA^{3}, Z=4, \mathrm{R}_{1}$ for 14342 [data intensity $I>2 \sigma(I)$ ] unique reflections = $0.0465, w \mathrm{R}_{2}$ (all 15191 unique reflections) $=0.0985$. CCDC ref: 1055218 .

Addition details of the structure solution and refinement: on initial refinement of the molecule as a $\mathrm{Sn}_{12}$ (boryl $)_{4}$ cluster some of the ADPs of the cluster atoms were noticeably enlarged, while others were markedly prolate. In order to better understand the problem, the occupancies were refined under various different conditions with different absorption corrections. The results of this in-depth study suggest that the cluster is in fact a disordered $\mathrm{Sn}_{11}$ (boryl $)_{4}$ system with two orientations. The geometry of the cluster is approximately icosahedral with one vacant site (Figure s22).
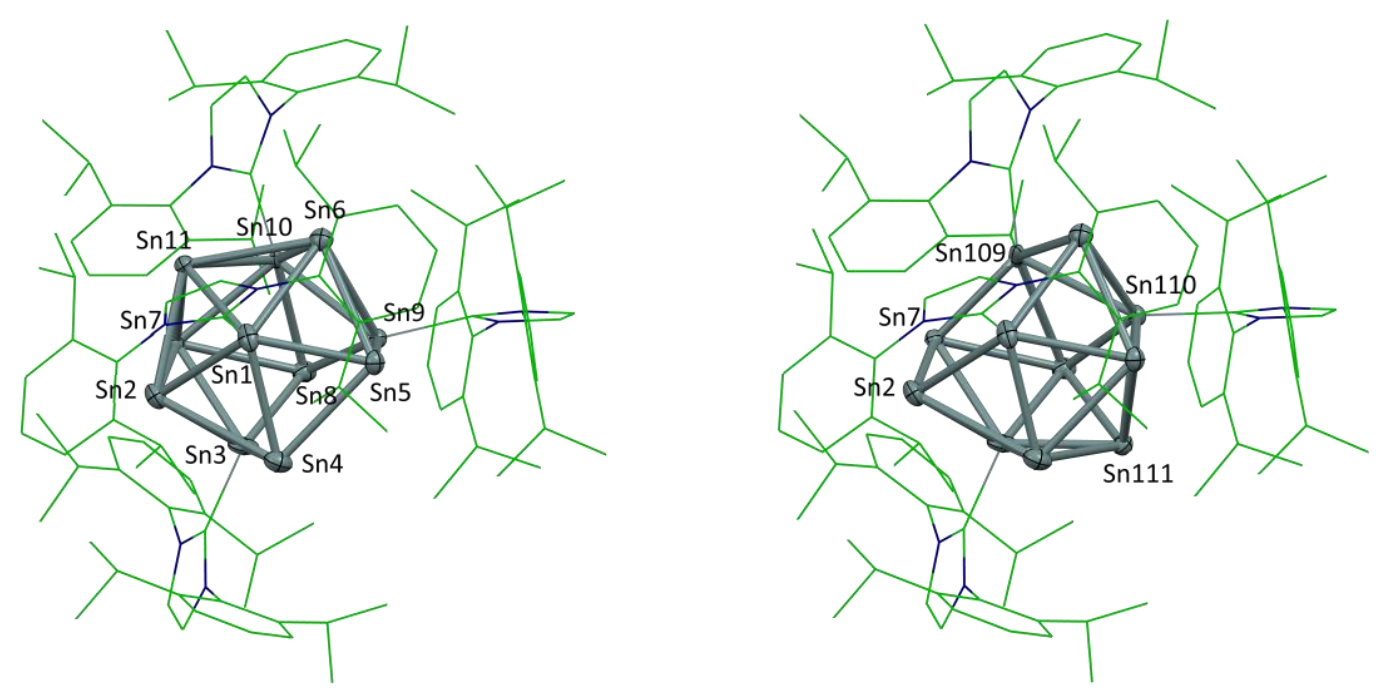

Figure s22: Two orientations of the (major) $\mathrm{Sn}_{11}$ cluster found in the solid state

On further refinement, it became apparent that two of the tin atoms exhibited enlarged ADPs with respect to the other tin atoms in the structure. On close examination, there was also a large residual electron density peak in the vicinity of these two atoms. Investigation suggested that a small amount of a $\mathrm{Sn}_{10}$ (boryl $)_{4}$ cluster was additionally present. Competitive refinement of this minor component indicated that the structure was approximately $80 \% \mathrm{Sn}_{11}$ and $20 \% \mathrm{Sn} 10$. 


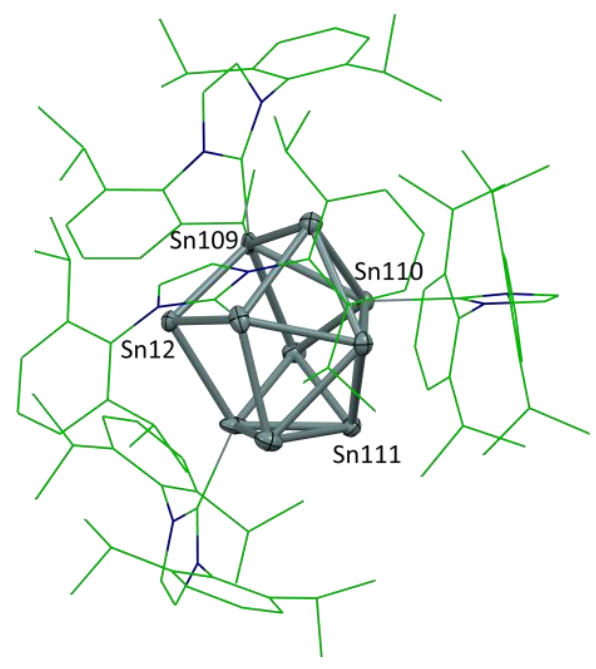

Figure s23: Minor $\mathrm{Sn}_{10}$ cluster component found in the solid state

The difference map also indicated the presence of diffuse electron density believed to be disordered hexane. In the early stages of the refinement, these were roughly modelled with poorly defined atoms to reduce the systematic error in the rest of the structure. In the latter stages, it was decided that the solvent was too ill-defined to treat with an atomic model, so SQUEEZE was used leaving a void from which the electron density was removed.

Crystal data for $\mathrm{Cl}_{2} \mathrm{Sn}\left\{\mathrm{B}(\mathrm{NDippCH})_{2}\right\}_{2}: a=11.3674(1) \AA ⿻$, $b=18.7715(1) \AA, c=$ 24.6570(2) A, orthorhombic, $P 22_{1} 2_{1} 2_{1}, V=5261.39(7) \AA^{3}, Z=4, \mathrm{R}_{1}$ for 11064 [data intensity $I>2 \sigma(I)]$ unique reflections $=0.0328, w \mathrm{R}_{2}$ (all 11978 unique reflections) $=$ 0.0391. CCDC ref: 1055213.

Crystal data for $\operatorname{Si}\left\{\operatorname{NDipp}\left(\mathrm{SiMe}_{3}\right)\right\}\left\{\operatorname{Si}\left(\mathrm{SiMe}_{3}\right)_{3}\right\}(\mathrm{H})\left(\mathrm{NH}_{2}\right): a=15.5841(2) \AA, \quad b=$ 12.2901(1) $\AA, c=18.1568(2) \AA, \beta=99.2133(10)^{\circ}$, monoclinic, $P 21 / n, V=3432.71(7)$ $\AA^{3}, Z=4, \mathrm{R}_{1}$ for 6306 [data intensity $I>2 \sigma(I)$ ] unique reflections $=0.0268, w \mathrm{R}_{2}($ all 7081 unique reflections $)=0.0303$. CCDC ref: 1055220 . 
Crystal data for $\mathrm{K}\left[\mathrm{B}\left(\mathrm{C}_{6} \mathrm{~F}_{5}\right)_{4}\right]: a=14.7357(4) \AA, b=9.6701(2) \AA, c=18.1354(5) \AA, \beta=$ 105.3864(5) ${ }^{\circ}$, monoclinic, $P 2_{1}, V=2362.01(12) \AA^{3}, Z=4, \mathrm{R}_{1}$ for 8939 [data intensity $I$ $>2 \sigma(I)]$ unique reflections $=0.0489, w \mathrm{R}_{2}$ (all 9391 unique reflections $)=0.1192$. CCDC ref: 1446878.

Crystal data for $\left\{\left(\mathrm{MeCCH}_{2}\right)_{2}\right\} \operatorname{Sn}\left\{\mathrm{NDipp}\left(\mathrm{SiMe}_{3}\right)\right\}\left\{\mathrm{B}(\mathrm{NDippCH})_{2}\right\}: a=11.3662(1) \AA$ $=19.9462(2) \AA, c=20.7654(3) \AA, b=91.344(1)^{\circ}$, monoclinic, $P 2_{1}, V=4706.5(1) \AA^{3}, Z$ $=4, \mathrm{R}_{1}$ for 16304 [data intensity $I>2 \sigma(I)$ ] unique reflections $=0.0483, w \mathrm{R}_{2}$ (all 16619 unique reflections) $=0.0518$. 


\section{DFT run files}

Run Files:

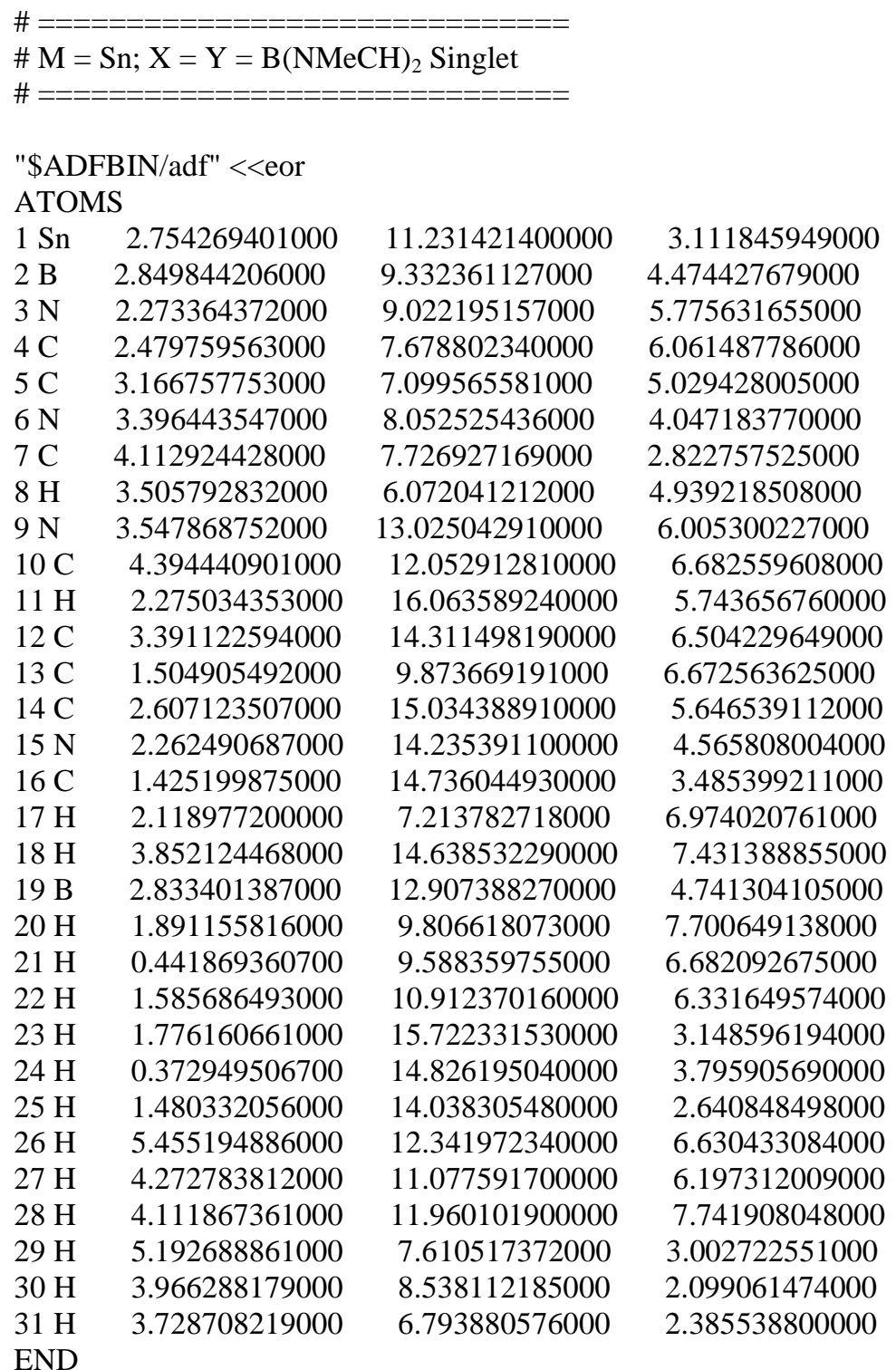

\section{GUIBONDS}

1123

21193

3261.0

4231.0

5341.0

63131.0

74171.0

8452.0

9581.0

10561.0

11671.0 1212142.0

1314151.0

1414111.0

1515161.0

1619151.0

171991.0

189121.0

199101.0

2012181.0

2120131.0 
2221131.0

2322131.0

2423161.0

2524161.0

2625161.0

2726101.0

2827101.0

2928101.0

302971.0

313071.0

323171.0

END

BASIS

type TZP

core Large

createoutput None

END

$\mathrm{XC}$

\# ==============================
\# $\mathrm{M}=\mathrm{Sn} ; \mathrm{X}=\mathrm{Y}=\mathrm{B}(\mathrm{NMeCH})_{2}$ Triplet
$\#$ =========================-

"\$ADFBIN/adf" <<eor

ATOMS

$1 \mathrm{Sn} \quad 2.807004815000$

2 B 2.733896838000

$3 \mathrm{~N} \quad 2.009130515000$

$4 \mathrm{C} \quad 2.233510167000$

$5 \mathrm{C} \quad 3.041972091000$

$6 \mathrm{~N} \quad 3.369265307000$

$7 \mathrm{C} \quad 4.217398551000$

$8 \mathrm{H} \quad 3.412126554000$

$9 \mathrm{~N} \quad 3.775171920000$

$10 \mathrm{C} \quad 4.734399377000$

$11 \mathrm{H} \quad 2.187615369000$

$12 \mathrm{C} \quad 3.535722625000$

$13 \mathrm{C} \quad 1.172878135000$

$14 \mathrm{C} \quad 2.602362865000$

$15 \mathrm{~N} \quad 2.207390757000$

$16 \mathrm{C} \quad 1.221174074000$

$17 \mathrm{H} \quad 1.808229534000$

$18 \mathrm{H} \quad 4.040247504000$

19 B 2.929230878000

$20 \mathrm{H} \quad 1.616523507000$

$21 \mathrm{H} \quad 0.170063379100$

$22 \mathrm{H} \quad 1.068495377000$

$23 \mathrm{H} \quad 1.600237095000$

$24 \mathrm{H} \quad 0.291149340000$

$25 \mathrm{H} \quad 0.990767966400$

$26 \mathrm{H} \quad 5.726891923000$

$27 \mathrm{H} \quad 4.820410515000$

$28 \mathrm{H} \quad 4.414606789000$
11.200348260000 9.085832519000

8.590304860000

7.217472767000

6.815764717000

7.918565117000

7.798451310000

5.818613076000

13.516526790000

12.701440490000

16.370027370000

14.848831490000

9.304602090000

15.367173300000

14.382838390000

14.643890870000

6.617973397000

15.338428160000

13.169658110000

9.299523216000

8.855524060000

10.345219100000

15.364188040000

15.041059460000

13.703422730000

13.176067400000

11.726514810000

12.538757480000
GGA Becke Perdew

END

RELATIVISTIC Scalar ZORA

SCANFREQ -1000 0

AnalyticalFreq

END

SAVE TAPE21 TAPE13

FULLSCF

INTEGRATION 6.0

NOPRINT LOGFILE

eor

3.528869774000

4.353844229000

5.501518437000

5.602987215000

4.578516276000

3.790308110000

2.615191732000

4.362369216000

5.772994633000

6.501497949000

5.239869265000

6.108164123000

6.453068220000

5.256732841000

4.351699884000

3.315244805000

6.401761149000

6.935222975000

4.653982589000

7.460399520000

6.509231658000

6.124826063000

2.574589068000

3.748521696000

2.800709074000

6.509581332000

6.007807415000

7.542064057000 


\begin{tabular}{|c|c|c|c|}
\hline \multicolumn{4}{|c|}{ 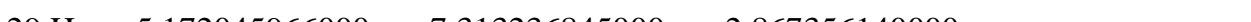 } \\
\hline $30 \mathrm{H}$ & 4.429896927000 & 8.801791538000 & 2.227323797000 \\
\hline $31 \mathrm{H}$ & 3.724119780000 & 7.214715061000 & 1.823289484000 \\
\hline \multicolumn{4}{|l|}{ END } \\
\hline \multicolumn{3}{|c|}{ GUIBONDS } & END \\
\hline 112 & & & \\
\hline \multicolumn{4}{|r|}{ CHARGE 0.02 .0} \\
\hline \multicolumn{4}{|c|}{3261.0} \\
\hline \multicolumn{4}{|r|}{ UNRESTRICTED } \\
\hline \multicolumn{4}{|c|}{5341.0} \\
\hline \multicolumn{4}{|c|}{63131.0} \\
\hline \multicolumn{4}{|c|}{74171.0} \\
\hline \multicolumn{4}{|r|}{ core Large } \\
\hline \multicolumn{4}{|r|}{ createoutput None } \\
\hline \multicolumn{4}{|c|}{10561.0} \\
\hline \multicolumn{4}{|c|}{11671.0} \\
\hline \multicolumn{4}{|c|}{1212142.0} \\
\hline \multicolumn{4}{|r|}{ GGA Becke Perdew } \\
\hline \multicolumn{4}{|c|}{1414111.0} \\
\hline \multicolumn{4}{|c|}{1515161.0} \\
\hline \multicolumn{4}{|r|}{ RELATIVISTIC Scalar ZORA } \\
\hline \multicolumn{4}{|c|}{171991.0} \\
\hline \multicolumn{4}{|c|}{189121.0} \\
\hline \multicolumn{4}{|c|}{199101.0} \\
\hline 2012 & 81.0 & & SCANFREQ -1000 0 \\
\hline 2120 & 31.0 & & \\
\hline 2221 & 31.0 & & AnalyticalFreq \\
\hline 2322 & 31.0 & & END \\
\hline 2423 & 61.0 & & \\
\hline 2524 & 61.0 & & SAVE TAPE21 TAPE13 \\
\hline 2625 & 61.0 & & \\
\hline 2726 & 01.0 & & FULLSCF \\
\hline 2827 & 01.0 & & INTEGRATION 6.0 \\
\hline 2928 & 01.0 & & \\
\hline 3029 & 1.0 & & NOPRINT LOGFILE \\
\hline 3130 & 1.0 & & \\
\hline 3231 & 1.0 & & eor \\
\hline$t===$ & -ー-ー-ー-ー & $==========$ & \\
\hline $\begin{array}{l}\# \mathrm{M}= \\
\#===\end{array}$ & $\begin{array}{l}\mathrm{n} ; \mathrm{X}=\mathrm{B}(\mathrm{NMeCH})_{2} \\
=============\end{array}$ & 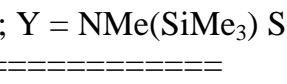 & nglet \\
\hline "\$AD & BIN/adf" <<eor & & \\
\hline ATOI & & & \\
\hline $1 \mathrm{Sn}$ & 10.485074060000 & 2.300128691000 & 2.394573286000 \\
\hline $2 \mathrm{~N}$ & 8.967740506000 & 3.814843022000 & 2.482699853000 \\
\hline $3 \mathrm{Si}$ & 7.937706004000 & 4.260299386000 & 3.861927188000 \\
\hline $4 \mathrm{C}$ & 8.986314821000 & 4.854902527000 & 5.319861225000 \\
\hline $5 \mathrm{C}$ & 6.883709312000 & 2.789986419000 & 4.415951307000 \\
\hline $6 \mathrm{C}$ & 6.769718598000 & 5.671143127000 & 3.355830217000 \\
\hline $7 \mathrm{C}$ & 8.780799143000 & 4.553457162000 & 1.220337638000 \\
\hline $8 \mathrm{H}$ & 6.100008625000 & 5.385813620000 & 2.530087522000 \\
\hline $9 \mathrm{H}$ & 8.545803702000 & -0.180758773700 & 3.386693338000 \\
\hline $10 \mathrm{H}$ & 13.287253870000 & 2.417352745000 & 5.950756336000 \\
\hline
\end{tabular}




\begin{tabular}{|c|c|c|c|}
\hline $11 \mathrm{H}$ & 6.211936802000 & 3.083135162000 & 5.239144966000 \\
\hline $12 \mathrm{C}$ & 11.114279750000 & 0.800170728100 & 6.736835593000 \\
\hline $13 \mathrm{C}$ & 10.184531830000 & -0.114322585600 & 6.342378098000 \\
\hline $14 \mathrm{~N}$ & 9.743040153000 & 0.210042199100 & 5.056651059000 \\
\hline $15 \mathrm{C}$ & 8.774898973000 & -0.623896446100 & 4.362438844000 \\
\hline $16 \mathrm{H}$ & 7.833184077000 & -0.700340300200 & 4.927068272000 \\
\hline $17 \mathrm{H}$ & 7.521851429000 & 1.970054329000 & 4.778233274000 \\
\hline $18 \mathrm{H}$ & 8.341628824000 & 5.174362644000 & 6.154868370000 \\
\hline $19 \mathrm{~B}$ & 10.420054480000 & 1.409037153000 & 4.608440911000 \\
\hline $20 \mathrm{~N}$ & 11.294236120000 & 1.736877832000 & 5.715373676000 \\
\hline $21 \mathrm{C}$ & 12.265002390000 & 2.811831040000 & 5.844846278000 \\
\hline $22 \mathrm{H}$ & 6.263597288000 & 2.410681016000 & 3.588812844000 \\
\hline $23 \mathrm{H}$ & 11.654695300000 & 0.852974906300 & 7.676832709000 \\
\hline $24 \mathrm{H}$ & 9.801970839000 & -0.969608027900 & 6.890637528000 \\
\hline $25 \mathrm{H}$ & 9.619909000000 & 5.708544551000 & 5.032807536000 \\
\hline $26 \mathrm{H}$ & 9.635652252000 & 4.045938162000 & 5.686721402000 \\
\hline $27 \mathrm{H}$ & 12.044990330000 & 3.448636808000 & 6.715231263000 \\
\hline $28 \mathrm{H}$ & 12.230443730000 & 3.443445181000 & 4.949968858000 \\
\hline $29 \mathrm{H}$ & 9.166343925000 & -1.639555026000 & 4.197882466000 \\
\hline $30 \mathrm{H}$ & 6.135812969000 & 5.940083604000 & 4.216381738000 \\
\hline $31 \mathrm{H}$ & 7.314353622000 & 6.578272162000 & 3.052011203000 \\
\hline $32 \mathrm{H}$ & 8.964502598000 & 5.634520503000 & 1.340960141000 \\
\hline $33 \mathrm{H}$ & 7.758742969000 & 4.440631153000 & 0.820479880100 \\
\hline $34 \mathrm{H}$ & 9.476529602000 & 4.186331471000 & 0.450235704000 \\
\hline \multicolumn{4}{|l|}{ END } \\
\hline \multicolumn{3}{|c|}{ GUIBONDS } & 3028211.0 \\
\hline \multicolumn{3}{|c|}{1121.0} & 3116151.0 \\
\hline \multicolumn{3}{|c|}{21193} & 323271.0 \\
\hline \multicolumn{3}{|c|}{3271.0} & 333371.0 \\
\hline \multicolumn{3}{|c|}{4231.0} & 343471.0 \\
\hline \multicolumn{3}{|c|}{5361.0} & END \\
\hline \multicolumn{3}{|c|}{6341.0} & \\
\hline \multicolumn{3}{|c|}{7351.0} & BASIS \\
\hline \multicolumn{3}{|c|}{84261.0} & type TZP \\
\hline \multicolumn{3}{|c|}{94181.0} & core Large \\
\hline \multicolumn{3}{|c|}{104251.0} & createoutput None \\
\hline \multicolumn{3}{|c|}{115221.0} & END \\
\hline \multicolumn{4}{|c|}{125111.0} \\
\hline \multicolumn{3}{|c|}{135171.0} & $\mathrm{XC}$ \\
\hline \multicolumn{3}{|c|}{14681.0} & GGA Becke Perdew \\
\hline \multirow{2}{*}{\multicolumn{3}{|c|}{$\begin{array}{llll}15 & 6 & 30 & 1.0 \\
16 & 6 & 31 & 1.0\end{array}$}} & END \\
\hline \multirow{2}{*}{\multicolumn{3}{|c|}{$\begin{array}{l}166311.0 \\
17 \quad 19201.0\end{array}$}} & \\
\hline & & & RELATIVISTIC Scalar ZORA \\
\hline \multicolumn{4}{|c|}{1829151.0} \\
\hline \multicolumn{4}{|c|}{199151.0} \\
\hline 2014 & 51.0 & & \\
\hline 2112 & 31.0 & & SCANFREQ -1000 0 \\
\hline 2210 & 11.0 & & \\
\hline 2327 & 11.0 & & AnalyticalFreq \\
\hline 2420 & 21.0 & & END \\
\hline 2520 & 11.0 & & \\
\hline 2612 & 32.0 & & SAVE TAPE21 TAPE13 \\
\hline 2713 & 41.0 & & \\
\hline 2813 & 41.0 & & FULLSCF \\
\hline 2919 & 41.0 & & INTEGRATION 6.0 \\
\hline
\end{tabular}




\begin{tabular}{|c|c|c|c|}
\hline$\# \mathrm{M}=$ & $\begin{array}{l}\mathrm{Sn} ; \mathrm{X}=\mathrm{B}(\mathrm{NMeCH}) \\
============\end{array}$ & $========$ & \\
\hline $\begin{array}{l}\text { "\$AD } \\
\text { ATOI }\end{array}$ & $\begin{array}{l}\text { "BIN/adf" <<eor } \\
\text { IS }\end{array}$ & & \\
\hline $1 \mathrm{Sn}$ & 10.674856540000 & 2.881499305000 & 3.227051430000 \\
\hline $2 \mathrm{~N}$ & 8.645096346000 & 3.267422346000 & 2.506434505000 \\
\hline $3 \mathrm{Si}$ & 7.688185724000 & 4.585932767000 & 3.211891474000 \\
\hline $4 \mathrm{C}$ & 8.205921255000 & 6.272823800000 & 2.513105907000 \\
\hline $5 \mathrm{C}$ & 7.906098274000 & 4.579395652000 & 5.087327627000 \\
\hline $6 \mathrm{C}$ & 5.858527129000 & 4.291586516000 & 2.792899426000 \\
\hline $7 \mathrm{C}$ & 8.390932627000 & 2.936023094000 & 1.096137118000 \\
\hline $8 \mathrm{H}$ & 5.521235797000 & 3.298158700000 & 3.127153658000 \\
\hline $9 \mathrm{H}$ & 8.014288913000 & 0.989607003700 & 3.620251381000 \\
\hline $10 \mathrm{H}$ & 13.774763740000 & 0.273994703700 & 4.972698709000 \\
\hline $11 \mathrm{H}$ & 556000 & 5401000 & 5.538990555000 \\
\hline $12 \mathrm{C}$ & 11.245477060000 & -0.726552073800 & 5.939357863000 \\
\hline $13 \mathrm{C}$ & 9.942797268000 & -0.953829389400 & 5.605371882000 \\
\hline $14 \mathrm{~N}$ & 239204000 & 333290 & 4.748186161000 \\
\hline $15 \mathrm{C}$ & 8.145663504000 & 0.076955105310 & 4.212996986000 \\
\hline $16 \mathrm{H}$ & 7.402275716000 & 0.073446081450 & 5.024470627000 \\
\hline $17 \mathrm{H}$ & 8.943472280000 & 4.797931299000 & 5.382588048000 \\
\hline $18 \mathrm{H}$ & 7.580213745000 & 7.079464438000 & 2.928498186000 \\
\hline $19 \mathrm{~B}$ & 10.59 & 550200 & 020000 \\
\hline $20 \mathrm{~N}$ & 11.687824270000 & 0.440105316400 & 5.309516730000 \\
\hline $21 \mathrm{C}$ & 13.045968600000 & 0.933103974500 & 5.468869154000 \\
\hline $22 \mathrm{H}$ & 7.631755779000 & 3.606690097000 & 5.522524736000 \\
\hline $23 \mathrm{H}$ & 11.888722150000 & -1.310891580000 & 6.589568535000 \\
\hline $24 \mathrm{H}$ & 9.295209501000 & -1.763653450000 & 5.926195549000 \\
\hline $25 \mathrm{H}$ & 8.106993199000 & 6.306214325000 & 1.416772820000 \\
\hline $26 \mathrm{H}$ & 9.255057954000 & 6.493296731000 & 2.765136306000 \\
\hline $27 \mathrm{H}$ & 13.311604270000 & 1.012839242000 & 6.533319010000 \\
\hline $28 \mathrm{H}$ & 13.123708900000 & 1.931834854000 & 5.023570497000 \\
\hline $29 \mathrm{H}$ & 7.962583090000 & -0.795699542700 & 3.567459841000 \\
\hline $30 \mathrm{H}$ & 5.235347168000 & 5.044682848000 & 3.301604381000 \\
\hline $31 \mathrm{H}$ & 5.658179990000 & 4.375727352000 & 1.713934292000 \\
\hline $32 \mathrm{H}$ & 8.630092219000 & 3.764307497000 & 0.404435932600 \\
\hline $33 \mathrm{H}$ & 7.325684383000 & 2.689126891000 & 0.947034961600 \\
\hline $34 \mathrm{H}$ & 8.984943003000 & 2.065266818000 & 0.782303165000 \\
\hline
\end{tabular}

\section{GUIBONDS}

1121.0

21193

3271.0

4231.0

5361.0

6341.0

7351.0

84261.0

94181.0

104251.0 115221.0 125111.0 135171.0 14681.0 156301.0 166311.0 1719201.0 1829151.0 199151.0 
2014151.0

2112231.0

2210211.0

2327211.0

2420121.0

2520211.0

2612132.0

2713241.0

2813141.0

2919141.0

3028211.0

3116151.0

323271.0

333371.0

343471.0

END

CHARGE 0.02 .0

UNRESTRICTED

\section{BASIS}

type TZP

core Large

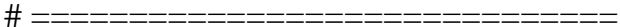

$\# \mathrm{M}=\mathrm{Sn} ; \mathrm{X}=\mathrm{B}(\mathrm{NMeCH})_{2} ; \mathrm{Y}=\mathrm{PMe}_{2}$ Singlet

\# ============================

"\$ADFBIN/adf" <<eor

ATOMS

$1 \mathrm{Sn} \quad 10.764683720000 \quad 2.315103405000$

$\begin{array}{llll}2 \mathrm{P} & 8.748615416000 & 3.783914004000 & 2.595532701000\end{array}$

$3 \mathrm{H} \quad 7.753003463000 \quad 3.266575119000 \quad 4.762403306000$

$4 \mathrm{H} \quad 12.975820680000 \quad 2.651012737000$

$5 \mathrm{H} \quad 9.188572885000 \quad-0.393121875400$

$6 \mathrm{H} \quad 8.306220810000 \quad-0.962303587000$

$7 \mathrm{C} \quad 7.967606705000 \quad 4.524167193000$

$8 \mathrm{H} \quad 7.109181901000 \quad 3.928308681000$

$9 \mathrm{H} \quad 8.108506679000 \quad 0.627837671700$

$10 \mathrm{H} \quad 13.561953930000 \quad 1.599088995000$

$11 \mathrm{H} \quad 8.718980513000 \quad 4.555951247000$

$12 \mathrm{C} \quad 11.021608730000$

$13 \mathrm{C} \quad 9.815294840000$

$14 \mathrm{~N} \quad 9.459911471000$

$15 \mathrm{C} \quad 8.246810684000$

$16 \mathrm{H} \quad 7.365892654000$

$17 \mathrm{H} \quad 11.580914190000$

$18 \mathrm{H} \quad 7.628962973000$

$19 \mathrm{~B} \quad 10.503273860000$

$20 \mathrm{~N} \quad 11.480719100000$

$21 \mathrm{C} \quad 12.742185520000$

$22 \mathrm{H} \quad 12.693063960000$

$23 \mathrm{C} \quad 7.366578583000$

$24 \mathrm{H} \quad 7.015176092000$

$25 \mathrm{H} \quad 6.521073071000$
0.804262416300

0.248043475200

0.624899296100

0.122468946700

0.322922869400

0.727561389300

5.549837149000

1.465870727000

1.539644171000

2.262278342000

3.113128467000

3.717557348000

4.736049404000

3.119857363000
4.672093567000

7.012248360000

4.299566790000

1.080632480000

0.738531053000

3.515060150000

5.984243440000

0.280856419600

6.711714072000

6.400148748000

5.104414454000

4.478021186000

5.106370332000

7.639011563000

1.286719519000

4.553509270000

5.619392565000

5.670097405000

6.366851360000

3.839491534000

4.060331193000

3.468719374000 createoutput None

END

$\mathrm{XC}$

GGA Becke Perdew

END

RELATIVISTIC Scalar ZORA

SCANFREQ -1000 0

AnalyticalFreq

END

SAVE TAPE21 TAPE13

FULLSCF

INTEGRATION 6.0

NOPRINT LOGFILE

eor 


$\begin{array}{llll}19 \mathrm{~B} & 10.650986400000 & 1.263561288000 & 4.651147474000 \\ 20 \mathrm{~N} & 11.263118940000 & 2.006380835000 & 5.727210626000 \\ 21 \mathrm{C} & 11.468661360000 & 3.437925592000 & 5.886628980000 \\ 22 \mathrm{H} & 10.985424400000 & 3.804883870000 & 6.804562649000 \\ 23 \mathrm{C} & 7.783022804000 & 4.960738423000 & 2.021104465000 \\ 24 \mathrm{H} & 7.685543578000 & 6.039623938000 & 1.824741772000 \\ 25 \mathrm{H} & 7.419901628000 & 4.405401770000 & 1.145530132000 \\ \text { END } & & & \end{array}$

\section{GUIBONDS}

1121.0

21193

325231.0

43231.0

51351.0

616151.0

722211.0

81871.0

9871.0

101171.0

1120211.0

1212132.0

1320121.0

1413141.0

1519141.0

164211.0

1719201.0

186151.0

199151.0

2014151.0

2112171.0

2210211.0

2324231.0

242321.0

25721.0

END

CHARGE 0.02 .0

\section{UNRESTRICTED}

\section{BASIS}

type TZP

core Large

createoutput None

END

$\mathrm{XC}$

GGA Becke Perdew

END

RELATIVISTIC Scalar ZORA

SCANFREQ -1000 0

AnalyticalFreq

END

SAVE TAPE21 TAPE13

FULLSCF

INTEGRATION 6.0

NOPRINT LOGFILE

eor

\footnotetext{
\# ==============================

$\# \mathrm{M}=\mathrm{Sn}^{+} ; \mathrm{X}=\mathrm{B}(\mathrm{NMeCH})_{2} ; \mathrm{L}=\mathrm{C}(\mathrm{NMeCH})_{2}$ Singlet

\# =============================

"\$ADFBIN/adf" <<eor ATOMS

$1 \mathrm{Sn} \quad 2.547654530000$

$2 \mathrm{C} \quad 2.893251550000$

11.209509140000

3.146694796000

$3 \mathrm{~N} \quad 2.203791412000$

9.333584658000

4.539758794000

4 C 2.516383936000

8.947038191000

5.651990043000

7.641328501000

5.994543588000

5 C 3.426998942000

7.201677488000

3.635857473000

8.243468425000

$7 \mathrm{C} \quad 4.563327945000$

8.187918253000

$8 \mathrm{H} \quad 3.937213445000$

6.249872322000

5.080882291000

4.192802829000

3.060341263000

4.994685579000

$9 \mathrm{~N} \quad 3.625594706000$

12.925721370000

6.003666839000

$\begin{array}{llll}10 \mathrm{C} & 4.363209257000 & 11.902474210000 & 6.739962363000\end{array}$
} 


\begin{tabular}{|c|c|c|c|}
\hline $11 \mathrm{H}$ & 2.700555354000 & 16.070097750000 & 5.596482190000 \\
\hline $12 \mathrm{C}$ & 3.604625340000 & 14.231573270000 & 6.441533723000 \\
\hline $13 \mathrm{C}$ & 1.207823068000 & 9.759820734000 & 6.355106458000 \\
\hline $14 \mathrm{C}$ & 2.909243365000 & 15.006136890000 & 5.541135299000 \\
\hline $15 \mathrm{~N}$ & 2.485536530000 & 14.218576880000 & 4.496457677000 \\
\hline $16 \mathrm{C}$ & 1.707710599000 & 14.783444250000 & 3.393981744000 \\
\hline $17 \mathrm{H}$ & 2.069776920000 & 7.138226095000 & 6.843789075000 \\
\hline $18 \mathrm{H}$ & 4.092084101000 & 14.548736970000 & 7.358399775000 \\
\hline $19 \mathrm{~B}$ & 2.908843711000 & 12.843184580000 & 4.736821378000 \\
\hline $20 \mathrm{H}$ & 1.291924940000 & 9.582017733000 & 7.432304820000 \\
\hline $21 \mathrm{H}$ & 0.196868170900 & 9.496773751000 & 6.020458579000 \\
\hline $22 \mathrm{H}$ & 1.404191427000 & 10.817528910000 & 6.146914345000 \\
\hline $23 \mathrm{H}$ & 2.205503850000 & 15.675959100000 & 2.993818224000 \\
\hline $24 \mathrm{H}$ & 0.697156885200 & 15.057654780000 & 3.725619689000 \\
\hline $25 \mathrm{H}$ & 1.619484557000 & 14.046721720000 & 2.587659465000 \\
\hline $26 \mathrm{H}$ & 5.438388071000 & 12.127330740000 & 6.748398369000 \\
\hline $27 \mathrm{H}$ & 4.213939862000 & 10.933502990000 & 6.254167002000 \\
\hline $28 \mathrm{H}$ & 4.010532316000 & 11.842448120000 & 7.778774275000 \\
\hline $29 \mathrm{H}$ & 5.562186323000 & 8.526331761000 & 3.360525385000 \\
\hline $30 \mathrm{H}$ & 4.181864781000 & 8.827915403000 & 2.255140586000 \\
\hline $31 \mathrm{H}$ & 4.620765425000 & 7.157683346000 & 2.695256366000 \\
\hline \multicolumn{4}{|l|}{ END } \\
\hline \multicolumn{3}{|c|}{ GUIBONDS } & 323171.0 \\
\hline \multicolumn{3}{|c|}{1121.0} & END \\
\hline \multicolumn{3}{|c|}{21191.0} & \\
\hline \multicolumn{3}{|c|}{3261.0} & CHARGE 1.0 \\
\hline \multicolumn{3}{|c|}{4231.0} & \\
\hline \multicolumn{3}{|c|}{5341.0} & BASIS \\
\hline \multicolumn{3}{|c|}{63131.0} & type TZP \\
\hline \multicolumn{3}{|c|}{74171.0} & core Large \\
\hline \multicolumn{3}{|c|}{8452.0} & createoutput None \\
\hline \multicolumn{3}{|c|}{9581.0} & END \\
\hline \multicolumn{4}{|c|}{10561.0} \\
\hline \multicolumn{3}{|c|}{11671.0} & $\mathrm{XC}$ \\
\hline \multicolumn{3}{|c|}{1212142.0} & GGA Becke Perdew \\
\hline \multicolumn{3}{|c|}{1314151.0} & END \\
\hline \multicolumn{3}{|c|}{1414111.0} & \\
\hline \multicolumn{3}{|c|}{1515161.0} & RELATIVISTIC Scalar ZORA \\
\hline \multicolumn{4}{|c|}{1619151.0} \\
\hline \multicolumn{4}{|c|}{171991.0} \\
\hline \multicolumn{4}{|c|}{189121.0} \\
\hline 1991 & 1.0 & & SCANFREQ -1000 0 \\
\hline 2012 & 31.0 & & \\
\hline 2120 & 31.0 & & AnalyticalFreq \\
\hline 2221 & 31.0 & & END \\
\hline 2322 & 31.0 & & \\
\hline 2423 & 51.0 & & SAVE TAPE21 TAPE13 \\
\hline 2524 & 51.0 & & \\
\hline 2625 & 51.0 & & FULLSCF \\
\hline 2726 & 1.0 & & INTEGRATION 6.0 \\
\hline 2827 & 1.0 & & \\
\hline 2928 & 1.0 & & NOPRINT LOGFILE \\
\hline 3029 & & & \\
\hline 3130 & 1.0 & & eor \\
\hline
\end{tabular}




\begin{tabular}{|c|c|c|c|}
\hline \multicolumn{4}{|c|}{$\begin{array}{l}\# \mathrm{M}=\mathrm{Sn}^{+} ; \mathrm{X}=\mathrm{B}(\mathrm{NMeCH})_{2} ; \mathrm{L}=\mathrm{C}(\mathrm{NMeCH})_{2} \text { Triplet } \\
\#==============================\end{array}$} \\
\hline \multicolumn{4}{|c|}{ "\$ADFBIN/adf" <<eor } \\
\hline \multicolumn{3}{|c|}{ ATOMS } & \\
\hline $2 \mathrm{C}$ & 2.567567980000 & $\begin{array}{l}11.141887820000 \\
9.219584971000\end{array}$ & 4.374703700000 \\
\hline $3 \mathrm{~N}$ & 2.063931698000 & 8.955889190000 & 5.615382494000 \\
\hline $4 \mathrm{C}$ & 1.759950688000 & 7.608616579000 & 5.734803085000 \\
\hline $5 \mathrm{C}$ & 2.082282667000 & 7.021621770000 & 4.548568192000 \\
\hline $6 \mathrm{~N}$ & 2.572673126000 & 8.019761921000 & 3.723910416000 \\
\hline $7 \mathrm{C}$ & 3.062812442000 & 7.805120623000 & 2.360710237000 \\
\hline $8 \mathrm{H}$ & 1.999535636000 & 5.990784603000 & 4.226285308000 \\
\hline $9 \mathrm{~N}$ & 3.813991380000 & 13.693932070000 & 5.605083729000 \\
\hline $10 \mathrm{C}$ & 5.191380165000 & 13.373369540000 & 5.977906506000 \\
\hline $11 \mathrm{H}$ & 1.108327890000 & 15.585591500000 & 5.804890931000 \\
\hline $12 \mathrm{C}$ & 3.148052374000 & 14.780513240000 & 6.135111946000 \\
\hline $13 \mathrm{C}$ & 1.859024325000 & 9.931713334000 & 6.686456490000 \\
\hline $14 \mathrm{C}$ & 1.890074274000 & 14.866889350000 & 5.579290901000 \\
\hline $15 \mathrm{~N}$ & 1.712929128000 & 13.839456080000 & 4.674916122000 \\
\hline $16 \mathrm{C}$ & 0.483607115900 & 13.697615080000 & 3.895706895000 \\
\hline $17 \mathrm{H}$ & 1.339173504000 & 7.186428436000 & 6.639493037000 \\
\hline $18 \mathrm{H}$ & 3.586135941000 & 15.416413870000 & 6.898124594000 \\
\hline $19 \mathrm{~B}$ & 2.899960454000 & 13.014630330000 & 4.714150677000 \\
\hline $20 \mathrm{H}$ & 2.464338683000 & 9.657598412000 & 7.558627262000 \\
\hline $21 \mathrm{H}$ & 0.799418934600 & 9.957743365000 & 6.966663199000 \\
\hline $22 \mathrm{H}$ & 2.162558216000 & 10.920885590000 & 6.328166391000 \\
\hline $23 \mathrm{H}$ & 0.330261030300 & 14.579763460000 & 3.260679094000 \\
\hline $24 \mathrm{H}$ & -0.385379552500 & 13.577560540000 & 4.556182012000 \\
\hline $25 \mathrm{H}$ & 0.567042259500 & 12.813382890000 & 3.254799393000 \\
\hline $26 \mathrm{H}$ & 5.871864413000 & 14.171482210000 & 5.653682341000 \\
\hline $27 \mathrm{H}$ & 5.486012026000 & 12.439181560000 & 5.487785403000 \\
\hline $28 \mathrm{H}$ & 5.280116900000 & 13.248201630000 & 7.065073054000 \\
\hline $29 \mathrm{H}$ & 4.159651527000 & 7.803427920000 & 2.344180796000 \\
\hline $30 \mathrm{H}$ & 2.685359911000 & 8.599557783000 & 1.705974251000 \\
\hline $31 \mathrm{H}$ & 2.691848507000 & 6.840992703000 & 2.000762403000 \\
\hline END & & & \\
\hline GUIB & DNDS & & 1619151.0 \\
\hline 112 & & & 171991.0 \\
\hline 2119 & & & 189121.0 \\
\hline 326 & & & 199101.0 \\
\hline 423 & & & 2012181.0 \\
\hline 534 & & & 2120131.0 \\
\hline 6313 & & & 2221131.0 \\
\hline 7417 & & & 2322131.0 \\
\hline 845 & & & 2423161.0 \\
\hline 958 & & & 2524161.0 \\
\hline 1056 & & & 2625161.0 \\
\hline 1167 & 1.0 & & 2726101.0 \\
\hline 1212 & 142.0 & & 2827101.0 \\
\hline 1314 & 151.0 & & $2928 \quad 101.0$ \\
\hline 1414 & 111.0 & & 302971.0 \\
\hline 1515 & 161.0 & & 313071.0 \\
\hline
\end{tabular}


323171.0

END

CHARGE 1.02 .0

UNRESTRICTED

BASIS

type TZP

core Large

createoutput None

END

$\mathrm{XC}$

GGA Becke Perdew

END

$\#========================$
$\# \mathrm{M}=\mathrm{Sn} ; \mathrm{X}=\mathrm{Y}=\mathrm{Si}\left(\mathrm{SiH}_{3}\right)_{3}$ Singlet

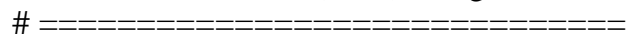

"\$ADFBIN/adf" <<eor ATOMS

$\begin{array}{llll}1 \mathrm{Sn} & 10.153971100000 & 2.032356907000 & 2.094995837000\end{array}$

$\begin{array}{llll}2 \mathrm{H} & 9.847771430000 & 5.332798516000 & 5.441615485000\end{array}$

$\begin{array}{llll}3 \mathrm{Si} & 12.703890800000 & 1.978086467000 & 5.226499422000\end{array}$

$\begin{array}{llll}4 \mathrm{Si} & 10.538990280000 & 1.173011398000 & 4.648106249000\end{array}$

$\begin{array}{llll}5 \mathrm{Si} & 9.088313445000 & 1.630208551000 & 6.467919439000\end{array}$

$\begin{array}{llll}6 \mathrm{H} & 9.641806555000 & 1.050071232000 & 7.737511201000\end{array}$

$\begin{array}{llll}7 \mathrm{H} & 12.644899420000 & 3.408728431000 & 5.673074153000\end{array}$

$\begin{array}{llll}8 \mathrm{H} & 8.901772811000 & 3.103207639000 & 6.667193091000\end{array}$

$\begin{array}{llll}9 \mathrm{H} & 7.744824939000 & 1.008153866000 & 6.227093473000\end{array}$

$10 \mathrm{Si} \quad 10.812069330000 \quad-1.179531586000 \quad 4.480422476000$

$\begin{array}{llll}11 \mathrm{H} & 9.562267809000 & -1.823775305000 & 3.953463331000\end{array}$

$\begin{array}{llll}12 \mathrm{H} & 11.128595580000 & -1.813060731000 & 5.803937538000\end{array}$

$\begin{array}{llll}13 \mathrm{H} & 13.304187440000 & 1.161018650000 & 6.332609959000\end{array}$

$\begin{array}{llll}14 \mathrm{H} & 13.631437600000 & 1.904418964000 & 4.047616992000\end{array}$

$\begin{array}{llll}15 \mathrm{H} & 11.925884560000 & -1.501143590000 & 3.526816762000\end{array}$

$\begin{array}{llll}16 \mathrm{Si} & 8.786994309000 & 4.351375352000 & 2.547800573000\end{array}$

$\begin{array}{llll}17 \mathrm{Si} & 8.596542752000 & 5.420047662000 & 0.438511836900\end{array}$

$\begin{array}{llll}18 \mathrm{H} & 7.836598977000 & 6.712879665000 & 0.505138886000\end{array}$

$19 \mathrm{H} \quad 9.958959084000 \quad 5.715681388000 \quad-0.119374070500$

$\begin{array}{llll}20 \mathrm{H} & 7.893524060000 & 4.518972091000 & -0.535413194600\end{array}$

$\begin{array}{llll}21 \mathrm{Si} & 6.578128458000 & 3.904647909000 & 3.298649219000\end{array}$

$\begin{array}{llll}22 \mathrm{H} & 5.942854517000 & 2.842453464000 & 2.449128153000\end{array}$

$23 \mathrm{H} \quad 5.719933054000 \quad 5.132794947000 \quad 3.211939342000$

$\begin{array}{llll}24 \mathrm{H} & 6.577182305000 & 3.423297635000 & 4.718395177000\end{array}$

$\begin{array}{llll}25 \mathrm{Si} & 9.792306853000 & 5.887491395000 & 4.050898004000\end{array}$

$\begin{array}{llll}26 \mathrm{H} & 11.193720570000 & 6.193992865000 & 3.610787218000\end{array}$

$\begin{array}{llll}27 \mathrm{H} & 9.029986389000 & 7.180196487000 & 4.084496387000\end{array}$

END

GUIBONDS
11611.0 


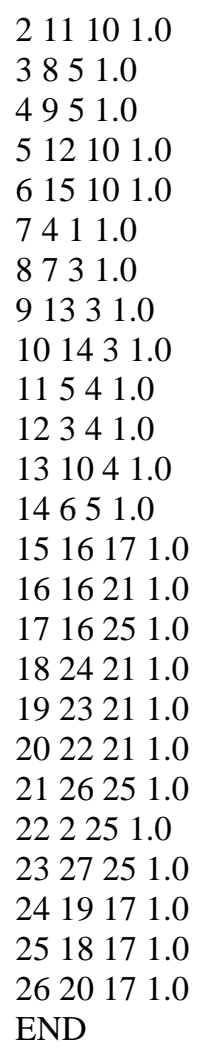

\section{BASIS}

type TZP

core Large

createoutput None

END

$\mathrm{XC}$

GGA Becke Perdew

END

RELATIVISTIC Scalar ZORA

SCANFREQ -1000 0

AnalyticalFreq

END

SAVE TAPE21 TAPE13

FULLSCF

INTEGRATION 6.0

NOPRINT LOGFILE

eor
2.625341174000
5.075518758000
4.752456541000
4.678062388000
6.679277500000
7.860768289000
5.053549706000
6.908226944000
6.590825691000
4.275805266000
4.150204200000
5.387125222000
5.816489594000
3.436441733000
2.997576615000
2.278212784000
$-0.018372201260$
$-0.304712205100$
$-0.406661583200$
$-0.863781045900$
2.825766850000 


\begin{tabular}{|c|c|c|c|}
\hline $22 \mathrm{H}$ & 6.231084802000 & 1.998632517000 & 1.919137178000 \\
\hline $23 \mathrm{H}$ & 5.248778891000 & 4.095055314000 & 2.678017508000 \\
\hline $24 \mathrm{H}$ & 6.466931186000 & 2.689497193000 & 4.242948796000 \\
\hline $25 \mathrm{Si}$ & 8.803056164000 & 6.206690874000 & 3.633153768000 \\
\hline $26 \mathrm{H}$ & 10.094199830000 & 6.862992424000 & 3.248302897000 \\
\hline $27 \mathrm{H}$ & 7.695220876000 & 7.210304937000 & 3.480975727000 \\
\hline \multicolumn{4}{|l|}{ END } \\
\hline \multicolumn{4}{|c|}{ GUIBONDS } \\
\hline \multicolumn{3}{|c|}{11611.0} & UNRESTRICTED \\
\hline \multicolumn{4}{|c|}{211101.0} \\
\hline \multicolumn{3}{|c|}{3851.0} & BASIS \\
\hline \multicolumn{3}{|c|}{4951.0} & type TZP \\
\hline \multicolumn{3}{|c|}{512101.0} & core Large \\
\hline \multicolumn{3}{|c|}{615101.0} & createoutput None \\
\hline \multicolumn{3}{|c|}{7411.0} & END \\
\hline \multicolumn{4}{|c|}{8731.0} \\
\hline \multicolumn{3}{|c|}{91331.0} & $\mathrm{XC}$ \\
\hline \multicolumn{3}{|c|}{101431.0} & GGA Becke Perdew \\
\hline \multicolumn{3}{|c|}{11541.0} & END \\
\hline \multicolumn{4}{|c|}{12341.0} \\
\hline \multicolumn{3}{|c|}{131041.0} & RELATIVISTIC Scalar ZORA \\
\hline \multicolumn{4}{|c|}{14651.0} \\
\hline \multicolumn{4}{|c|}{1516171.0} \\
\hline \multicolumn{4}{|c|}{1616211.0} \\
\hline \multirow{2}{*}{\multicolumn{4}{|c|}{$\begin{array}{llll}17 & 16 & 25 & 1.0 \\
18 & 24 & 21 & 1.0\end{array}$}} \\
\hline & \multicolumn{3}{|c|}{1824211.0} \\
\hline \multicolumn{3}{|c|}{1923211.0} & AnalyticalFreq \\
\hline \multirow{2}{*}{\multicolumn{4}{|c|}{$\begin{array}{llll}20 & 22 & 21 & 1.0 \\
21 & 26 & 25 & 1.0\end{array}$}} \\
\hline & 51.0 & & \\
\hline \multicolumn{3}{|c|}{222251.0} & SAVE TAPE21 TAPE13 \\
\hline 2327 & 51.0 & & \\
\hline 2419 & 71.0 & & FULLSCF \\
\hline 2518 & 71.0 & & INTEGRATION 6.0 \\
\hline 2620 & 71.0 & & \\
\hline END & & & NOPRINT LOGFILE \\
\hline CHA & GE 0.02 .0 & & eor \\
\hline$\#===$ & $==============$ & $==========$ & \\
\hline $\begin{array}{l}\# \mathrm{M}= \\
\#===\end{array}$ & $\begin{array}{l}\mathrm{Sn} ; \mathrm{X}=\mathrm{Si}\left(\mathrm{SiH}_{3}\right)_{3} ; \mathrm{Y} \\
=========\end{array}$ & $\begin{array}{l}=\mathrm{NMe}\left(\mathrm{SiMe}_{3}\right) \text { Sing } \\
=========\end{array}$ & \\
\hline $\begin{array}{l}\text { "\$AD } \\
\text { ATOI }\end{array}$ & $\begin{array}{l}\text { BIN/adf" <<eor } \\
\text { S }\end{array}$ & & \\
\hline $1 \mathrm{Sn}$ & 10.509414030000 & 2.465682685000 & 2.299935859000 \\
\hline $2 \mathrm{~N}$ & 8.922559166000 & 3.892772497000 & 2.309770846000 \\
\hline $3 \mathrm{Si}$ & 7.758719133000 & 4.311547201000 & 3.595246614000 \\
\hline $4 \mathrm{C}$ & 8.647965262000 & 5.253809190000 & 4.974038945000 \\
\hline $5 \mathrm{C}$ & 6.951683865000 & 2.739647140000 & 4.258246954000 \\
\hline $6 \mathrm{C}$ & 6.398364635000 & 5.421018769000 & 2.875605712000 \\
\hline $7 \mathrm{C}$ & 8.870037666000 & 4.681957974000 & 1.062675112000 \\
\hline $8 \mathrm{H}$ & 5.848266341000 & 4.923700429000 & 2.061844264000 \\
\hline $9 \mathrm{H}$ & 5.669819297000 & 5.658223393000 & 3.667833999000 \\
\hline
\end{tabular}




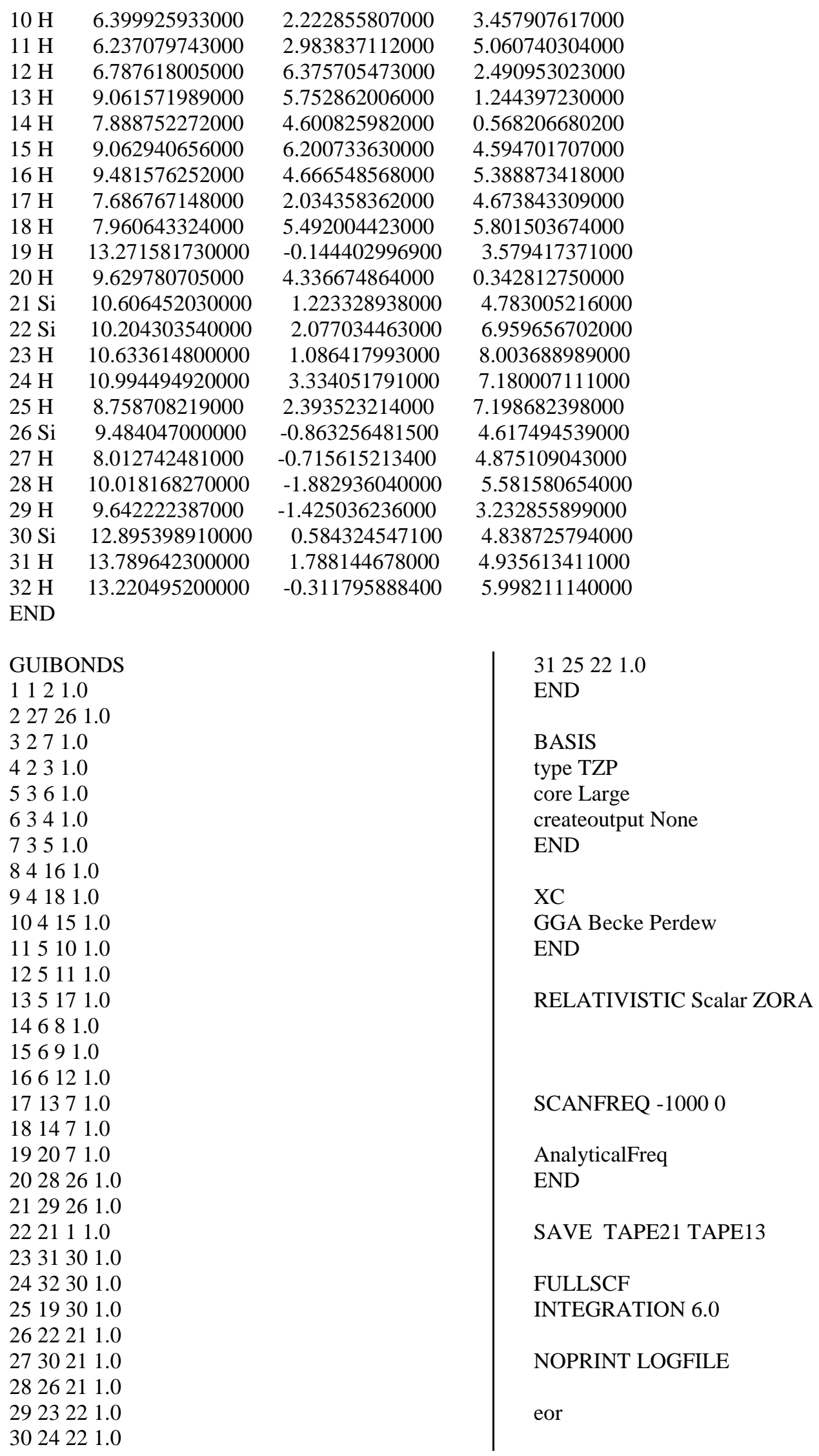




\begin{tabular}{|c|c|c|c|}
\hline \multicolumn{4}{|c|}{$\begin{array}{l}\text { \# } \mathrm{M}=\mathrm{Sn} ; \mathrm{X}=\mathrm{Si}\left(\mathrm{SiH}_{3}\right)_{2} ; \mathrm{Y}=\mathrm{NMe}\left(\mathrm{SiMe}_{3}\right) \text { Triplet } \\
\#========================\end{array}$} \\
\hline \multicolumn{4}{|c|}{ "\$ADFBIN/adf" <<eor } \\
\hline $1 \mathrm{Sn}$ & 10.589937350000 & 3.162391039000 & 2.993660747000 \\
\hline $2 \mathrm{~N}$ & 8.547169332000 & 3.798454347000 & 3.495181648000 \\
\hline $3 \mathrm{Si}$ & 7.169528300000 & 3.385601406000 & 2.444074073000 \\
\hline $4 \mathrm{C}$ & 5.575472964000 & 3.539782678000 & 3.460776716000 \\
\hline $5 \mathrm{C}$ & 7.367050454000 & 1.612248936000 & 1.832407560000 \\
\hline $6 \mathrm{C}$ & 7.081830058000 & 4.572022271000 & 0.966499278500 \\
\hline $7 \mathrm{C}$ & 8.422027881000 & 4.997639243000 & 4.331467906000 \\
\hline $8 \mathrm{H}$ & 7.981596368000 & 4.481763426000 & 0.338205317100 \\
\hline $9 \mathrm{H}$ & 6.205404430000 & 4.345384550000 & 0.338056955100 \\
\hline $10 \mathrm{H}$ & 8.257833788000 & 1.490053361000 & 1.197710869000 \\
\hline $11 \mathrm{H}$ & 6.486992353000 & 1.325225544000 & 1.234013316000 \\
\hline $12 \mathrm{H}$ & 6.997309160000 & 5.620885667000 & 1.290731303000 \\
\hline $13 \mathrm{H}$ & 7.572725528000 & 4.888702737000 & 5.028083706000 \\
\hline $14 \mathrm{H}$ & 8.239366762000 & 5.914840577000 & 3.741909763000 \\
\hline $15 \mathrm{H}$ & 5.378843495000 & 4.576933794000 & 3.771896714000 \\
\hline $16 \mathrm{H}$ & 5.611932454000 & 2.911074747000 & 4.363577608000 \\
\hline $17 \mathrm{H}$ & 7.449499554000 & 0.905705534300 & 2.672022358000 \\
\hline $18 \mathrm{H}$ & 4.716364961000 & 3.208596870000 & 2.855557262000 \\
\hline $19 \mathrm{H}$ & 9.880861752000 & -1.210644805000 & 2.858709541000 \\
\hline $20 \mathrm{H}$ & 9.329388930000 & 5.162517748000 & 4.929924287000 \\
\hline $21 \mathrm{Si}$ & 11.088229260000 & 1.105640961000 & 4.725092912000 \\
\hline $22 \mathrm{Si}$ & 13.167655220000 & 1.606985806000 & 5.742241680000 \\
\hline $23 \mathrm{H}$ & 13.692141300000 & 0.454722752600 & 6.551789896000 \\
\hline $24 \mathrm{H}$ & 14.185337960000 & 1.930627893000 & 4.689352006000 \\
\hline $25 \mathrm{H}$ & 13.026082460000 & 2.795368490000 & 6.644923169000 \\
\hline $26 \mathrm{Si}$ & 9.349109537000 & 1.024272510000 & 6.328938257000 \\
\hline $27 \mathrm{H}$ & 9.222074624000 & 2.338529301000 & 7.037452612000 \\
\hline $28 \mathrm{H}$ & 9.616330363000 & -0.035550900570 & 7.359934678000 \\
\hline $29 \mathrm{H}$ & 8.042001570000 & 0.705526579000 & 5.669637836000 \\
\hline $30 \mathrm{Si}$ & 11.211766250000 & -0.906123438800 & 3.477691578000 \\
\hline $31 \mathrm{H}$ & 12.221513080000 & -0.759092824200 & 2.379062343000 \\
\hline $32 \mathrm{H}$ & 11.616031160000 & -2.079283331000 & 4.326718841000 \\
\hline \multicolumn{4}{|l|}{ END } \\
\hline \multicolumn{3}{|c|}{ GUIBONDS } & 14681.0 \\
\hline \multicolumn{3}{|c|}{1121.0} & 15691.0 \\
\hline \multicolumn{3}{|c|}{227261.0} & 166121.0 \\
\hline \multicolumn{3}{|c|}{3271.0} & 171371.0 \\
\hline \multicolumn{3}{|c|}{4231.0} & 181471.0 \\
\hline \multicolumn{3}{|c|}{5361.0} & 192071.0 \\
\hline \multicolumn{3}{|c|}{6341.0} & 2028261.0 \\
\hline \multicolumn{3}{|c|}{7351.0} & 2129261.0 \\
\hline \multicolumn{3}{|c|}{84161.0} & 222111.0 \\
\hline \multicolumn{3}{|c|}{94181.0} & 2331301.0 \\
\hline \multicolumn{3}{|c|}{104151.0} & 2432301.0 \\
\hline \multicolumn{3}{|c|}{115101.0} & 2519301.0 \\
\hline \multicolumn{3}{|c|}{125111.0} & 2622211.0 \\
\hline \multicolumn{3}{|c|}{135171.0} & 2730211.0 \\
\hline
\end{tabular}


2826211.0

2923221.0

3024221.0

3125221.0

END

CHARGE 0.0 2.0

\section{UNRESTRICTED}

BASIS
type TZP
core Large
createoutput None
END
XC
GGA Becke Perdew
END

\begin{tabular}{|c|c|c|c|}
\hline \multicolumn{4}{|c|}{ 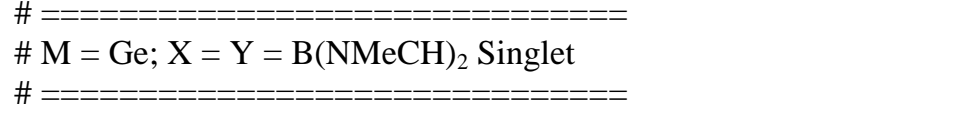 } \\
\hline \multicolumn{4}{|c|}{ "\$ADFBIN/adf" <<eor } \\
\hline $1 \mathrm{Ge}$ & 2.763547203000 & 11.226823750000 & 3.177520221000 \\
\hline $2 \mathrm{~B}$ & 2.815368544000 & 9.502412332000 & 4.440084400000 \\
\hline $3 \mathrm{~N}$ & 2.208872810000 & 9.191695704000 & 5.732776555000 \\
\hline $4 \mathrm{C}$ & 2.445029400000 & 7.860448751000 & 6.044206896000 \\
\hline $5 \mathrm{C}$ & 3.186411778000 & 7.287214369000 & 5.045542515000 \\
\hline $6 \mathrm{~N}$ & 3.411924030000 & 8.229294332000 & 4.054209591000 \\
\hline $7 \mathrm{C}$ & 4.180567261000 & 7.915224861000 & 2.859391671000 \\
\hline $8 \mathrm{H}$ & 3.565877972000 & 6.271403582000 & 4.988190568000 \\
\hline $9 \mathrm{~N}$ & 3.604545383000 & 12.866668910000 & 5.931308562000 \\
\hline $10 \mathrm{C}$ & 4.501608125000 & 11.918309100000 & 6.577246975000 \\
\hline $11 \mathrm{H}$ & 2.198217753000 & 15.853636870000 & 5.775688576000 \\
\hline $12 \mathrm{C}$ & 3.413580139000 & 14.135427920000 & 6.459235311000 \\
\hline $13 \mathrm{C}$ & 1.393652492000 & 10.027272870000 & 22531000 \\
\hline $14 \mathrm{C}$ & 2.573953174000 & 14.843724400000 & 5.641331983000 \\
\hline $15 \mathrm{~N}$ & 2.236040137000 & 14.056404340000 & 4.551838819000 \\
\hline $16 \mathrm{C}$ & 1.345921651000 & 14.536924210000 & 3.505959221000 \\
\hline $17 \mathrm{H}$ & 2.068009825000 & & 6.951742272000 \\
\hline $18 \mathrm{H}$ & 3.889057681000 & 14.461922050000 & 7.379572304000 \\
\hline $19 \mathrm{~B}$ & 2.861024218000 & 12.745350710000 & 4.679136008000 \\
\hline $20 \mathrm{H}$ & 1.746794670000 & 9.963380883000 & 7.643966292000 \\
\hline $21 \mathrm{H}$ & 0.336560617200 & 9.721634616000 & 6.577798551000 \\
\hline $22 \mathrm{H}$ & 1.468266509000 & 11.067912190000 & 6.268721668000 \\
\hline $23 \mathrm{H}$ & 1.634272349000 & 15.549255240000 & 3.188092970000 \\
\hline $24 \mathrm{H}$ & 0.298462750100 & 14.561194190000 & 3.843671447000 \\
\hline $25 \mathrm{H}$ & 1.418515811000 & 13.860808520000 & 2.645224117000 \\
\hline $26 \mathrm{H}$ & 5.552181421000 & 12.233212150000 & 6.484231330000 \\
\hline $27 \mathrm{H}$ & 4.383777193000 & 10.938025680000 & 6.101984566000 \\
\hline $28 \mathrm{H}$ & 4.261933630000 & 11.824852750000 & 7.647160883000 \\
\hline $29 \mathrm{H}$ & 5.258058191000 & 7.849974933000 & 3.075199110000 \\
\hline
\end{tabular}

RELATIVISTIC Scalar ZORA

SCANFREQ -1000 0

AnalyticalFreq

END

SAVE TAPE21 TAPE13

FULLSCF

INTEGRATION 6.0

NOPRINT LOGFILE

eor 


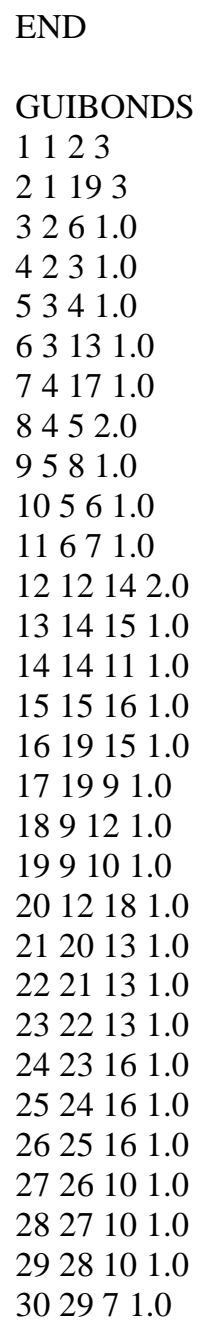

$\begin{array}{ll}8.710081636000 & 2.120428873000 \\ 6.958429723000 & 2.428798201000\end{array}$

$\begin{array}{ll}8.710081636000 & 2.120428873000 \\ 6.958429723000 & 2.428798201000\end{array}$

313071.0

323171.0

END

\section{BASIS}

type TZP

core Large

createoutput None

END

$\mathrm{XC}$

GGA Becke Perdew

END

RELATIVISTIC Scalar ZORA

SCANFREQ -1000 0

AnalyticalFreq

END

SAVE TAPE21 TAPE13

FULLSCF

INTEGRATION 6.0

NOPRINT LOGFILE

eor

\begin{abstract}
3.716165811000
4.419128028000

5.590452698000

5.648043299000

4.576026303000

3.799620945000

2.583254650000

4.320758214000

5.845234528000

6.645628149000

5.136537647000

6.126095820000

6.590934327000
\end{abstract}




$\begin{array}{lccc}14 \mathrm{C} & 2.650852417000 & 15.225685210000 & 5.205800448000 \\ 15 \mathrm{~N} & 2.268927009000 & 14.231091360000 & 4.308742149000 \\ 16 \mathrm{C} & 1.357212851000 & 14.491221150000 & 3.206326753000 \\ 17 \mathrm{H} & 1.922727040000 & 6.733601727000 & 6.451500827000 \\ 18 \mathrm{H} & 3.987828603000 & 15.193594820000 & 6.966270930000 \\ 19 \mathrm{~B} & 2.921124928000 & 12.996806740000 & 4.686129058000 \\ 20 \mathrm{H} & 1.768436002000 & 9.385330991000 & 7.580783835000 \\ 21 \mathrm{H} & 0.284794514000 & 8.975288149000 & 6.673819830000 \\ 22 \mathrm{H} & 1.179657204000 & 10.468978960000 & 6.292118728000 \\ 23 \mathrm{H} & 1.805501384000 & 15.173399100000 & 2.468029175000 \\ 24 \mathrm{H} & 0.417047321600 & 14.933612710000 & 3.568299457000 \\ 25 \mathrm{H} & 1.126359248000 & 13.541663140000 & 2.708942958000 \\ 26 \mathrm{H} & 5.612183679000 & 12.951028260000 & 6.690816106000 \\ 27 \mathrm{H} & 4.669030157000 & 11.525363430000 & 6.185280968000 \\ 28 \mathrm{H} & 4.224602130000 & 12.402485280000 & 7.673437732000 \\ 29 \mathrm{H} & 5.122509664000 & 7.530581782000 & 2.778463806000 \\ 30 \mathrm{H} & 4.330058349000 & 9.022481893000 & 2.207990364000 \\ 31 \mathrm{H} & 3.626995537000 & 7.433681196000 & 1.803832557000 \\ \mathrm{END} & & & \end{array}$

\section{GUIBONDS}

1123

21193

3261.0

4231.0

5341.0

63131.0

74171.0

8452.0

9581.0

10561.0

11671.0

1212142.0

1314151.0

1414111.0

1515161.0

1619151.0

171991.0

189121.0

199101.0

2012181.0

2120131.0

2221131.0

2322131.0

2423161.0

2524161.0

2625161.0

2726101.0

2827101.0

2928101.0

302971.0

313071.0

323171.0
END

CHARGE 0.02 .0

UNRESTRICTED

\section{BASIS}

type TZP

core Large

createoutput None

END

$\mathrm{XC}$

GGA Becke Perdew

END

RELATIVISTIC Scalar ZORA

SCANFREQ -1000 0

AnalyticalFreq

END

SAVE TAPE21 TAPE13

FULLSCF

INTEGRATION 6.0

NOPRINT LOGFILE

eor

\# 


\begin{tabular}{|c|c|c|c|}
\hline \multicolumn{4}{|c|}{ "\$ADFBIN/adf" <<eor } \\
\hline \multicolumn{4}{|c|}{ ATOMS } \\
\hline $1 \mathrm{Ge}$ & 5.519166611000 & 6.955572507000 & 4.237811355000 \\
\hline $2 \mathrm{Si}$ & 3.208083387000 & 7.064583232000 & 6.717249848000 \\
\hline $3 \mathrm{~N}$ & 7.310365316000 & 5.655651209000 & 6.659874855000 \\
\hline $4 \mathrm{~N}$ & 7.457451949000 & 7.924482217000 & 6.702140990000 \\
\hline $5 \mathrm{~N}$ & 3.726135251000 & 7.063110111000 & 5.000221033000 \\
\hline $6 \mathrm{C}$ & 8.241349542000 & 6.033998265000 & 7.632049784000 \\
\hline $7 \mathrm{H}$ & 8.770876094000 & 5.309983496000 & 8.243718757000 \\
\hline $8 \mathrm{C}$ & 8.329542236000 & 7.393337672000 & 7.657308069000 \\
\hline $9 \mathrm{H}$ & 8.946736471000 & 8.019788971000 & 8.293970919000 \\
\hline $10 \mathrm{C}$ & 7.040398430000 & 4.253884794000 & 6.381148743000 \\
\hline $11 \mathrm{H}$ & 6.693676561000 & 3.726223580000 & 7.282966687000 \\
\hline $12 \mathrm{H}$ & 6.602038018000 & 9.562890015000 & 5.723878240000 \\
\hline $13 \mathrm{H}$ & 7.937319083000 & 3.741179953000 & 6.001474295000 \\
\hline $14 \mathrm{H}$ & 7.097222201000 & 9.894192096000 & 7.396473957000 \\
\hline $15 \mathrm{H}$ & 8.329678131000 & 9.763315296000 & 6.110912991000 \\
\hline $16 \mathrm{H}$ & 6.251977264000 & 4.179256836000 & 5.624045455000 \\
\hline $17 \mathrm{C}$ & 7.373026361000 & 9.358662278000 & 6.475025515000 \\
\hline $18 \mathrm{C}$ & 1.312169157000 & 7.182375975000 & 6.803906978000 \\
\hline $19 \mathrm{H}$ & 4.804394008000 & 5.386182273000 & 7.681570198000 \\
\hline $20 \mathrm{C}$ & 3.905783551000 & 8.550611713000 & 7.655439034000 \\
\hline $21 \mathrm{H}$ & 5.000749934000 & 8.494377724000 & 7.735015010000 \\
\hline $22 \mathrm{~B}$ & 6.760672612000 & 6.842458975000 & 6.038542763000 \\
\hline $23 \mathrm{H}$ & 3.493321956000 & 8.569876553000 & 8.677762861000 \\
\hline $24 \mathrm{H}$ & 0.920667406800 & 8.107289970000 & 6.353608292000 \\
\hline $25 \mathrm{H}$ & 3.636855903000 & 9.498844470000 & 7.164828238000 \\
\hline $26 \mathrm{C}$ & 3.711304324000 & 5.471227417000 & 7.601669040000 \\
\hline $27 \mathrm{H}$ & 3.326248731000 & 4.582115892000 & 7.078828630000 \\
\hline $28 \mathrm{H}$ & 3.296542117000 & 5.468830719000 & 8.623246058000 \\
\hline $29 \mathrm{H}$ & 1.012958083000 & 7.182807966000 & 7.865189372000 \\
\hline $30 \mathrm{H}$ & 0.808838852200 & 6.329852551000 & 6.322862984000 \\
\hline $31 \mathrm{C}$ & 2.656514847000 & 7.159939180000 & 3.987741833000 \\
\hline $32 \mathrm{H}$ & 3.092409410000 & 7.146891560000 & 2.977973222000 \\
\hline $33 \mathrm{H}$ & 2.075369736000 & 8.090819710000 & 4.091284847000 \\
\hline $34 \mathrm{H}$ & 1.945817849000 & 6.320531200000 & 4.058710411000 \\
\hline END & & & \\
\hline GUIB & NDS & & 15682.0 \\
\hline 115 & & & 16891.0 \\
\hline 2122 & & & 1718301.0 \\
\hline 325 & & & 1820251.0 \\
\hline 4226 & & & 1915171.0 \\
\hline 5218 & & & 2013101.0 \\
\hline 6220 & & & 2120231.0 \\
\hline 736 & & & 2214171.0 \\
\hline 8310 & & & 2318291.0 \\
\hline 9322 & & & 2416101.0 \\
\hline 104 & & & 2518241.0 \\
\hline 114 & 1.0 & & 2620211.0 \\
\hline 1242 & 1.0 & & 2712171.0 \\
\hline 1353 & 1.0 & & 2811101.0 \\
\hline $146^{7}$ & & & 2926281.0 \\
\hline
\end{tabular}


3026271.0

3126191.0

3232311.0

3333311.0

3434311.0

END

BASIS

type TZP

core Large

createoutput None

END

$\mathrm{XC}$

GGA Becke Perdew

END
RELATIVISTIC Scalar ZORA

SCANFREQ -1000 0

AnalyticalFreq

END

SAVE TAPE21 TAPE13

FULLSCF

INTEGRATION 6.0

NOPRINT LOGFILE

eor

\begin{tabular}{|c|c|c|c|}
\hline \multicolumn{4}{|c|}{$\begin{array}{l}\# \mathrm{M}=\mathrm{Ge} ; \mathrm{X}=\mathrm{B}(\mathrm{NMeCH})_{2} ; \mathrm{Y}=\mathrm{NMe}\left(\mathrm{SiMe}_{3}\right) \text { Triplet } \\
\# \text { =========================== }\end{array}$} \\
\hline \multicolumn{4}{|c|}{ "\$ADFBIN/adf" <<eor } \\
\hline $1 \mathrm{Ge}$ & 5.372878586000 & 5.909136208000 & 5.282435447000 \\
\hline $2 \mathrm{Si}$ & 2.595149619000 & 7.276921101000 & 6.397681825000 \\
\hline $3 \mathrm{~N}$ & 8.400788731000 & 6.194071454000 & 6.120135730000 \\
\hline $4 \mathrm{~N}$ & 7.350946445000 & 8.210851545000 & 6.424193821000 \\
\hline $5 \mathrm{~N}$ & 3.921906266000 & 7.266500864000 & 5.212616701000 \\
\hline $6 \mathrm{C}$ & 9.320503614000 & 7.124813433000 & 6.607420207000 \\
\hline $7 \mathrm{H}$ & 10.359157040000 & 6.869751345000 & 6.792258204000 \\
\hline $8 \mathrm{C}$ & 8.695929672000 & 8.324457015000 & 6.787861464000 \\
\hline $9 \mathrm{H}$ & 9.116077281000 & 9.256538666000 & 7.152781960000 \\
\hline $10 \mathrm{C}$ & 8.773111121000 & 4.823923574000 & 5.812037089000 \\
\hline $11 \mathrm{H}$ & 9.180205373000 & 4.314226099000 & 6.698143577000 \\
\hline $12 \mathrm{H}$ & 5.450035543000 & 9.012635545000 & 6.160941822000 \\
\hline $13 \mathrm{H}$ & 9.523262701000 & 4.785361903000 & 5.007546563000 \\
\hline $14 \mathrm{H}$ & 6.343552732000 & 9.691776598000 & 7.551307586000 \\
\hline $15 \mathrm{H}$ & 6.777953329000 & 10.175328450000 & 5.887774667000 \\
\hline $16 \mathrm{H}$ & 7.884682366000 & 4.275511033000 & 5.478910534000 \\
\hline $17 \mathrm{C}$ & 6.435271079000 & 9.336712649000 & 6.513090082000 \\
\hline $18 \mathrm{C}$ & 1.982542470000 & 9.065812093000 & 6.591263656000 \\
\hline $19 \mathrm{H}$ & 1.444558849000 & 5.157655388000 & 5.694648826000 \\
\hline $20 \mathrm{C}$ & 3.237433447000 & 6.658862394000 & 8.058804197000 \\
\hline $21 \mathrm{H}$ & 3.556617040000 & 5.607439811000 & 8.010943444000 \\
\hline $22 \mathrm{~B}$ & 7.125353994000 & 6.853507180000 & 5.987595693000 \\
\hline $23 \mathrm{H}$ & 2.440604652000 & 6.739725200000 & 8.816406414000 \\
\hline $24 \mathrm{H}$ & 2.793824458000 & 9.744878292000 & 6.895420030000 \\
\hline $25 \mathrm{H}$ & 4.096384871000 & 7.252802984000 & 8.405221256000 \\
\hline $26 \mathrm{C}$ & 1.140941748000 & 6.209222649000 & 5.813503373000 \\
\hline $27 \mathrm{H}$ & 0.743148022600 & 6.558789199000 & 4.847879465000 \\
\hline $28 \mathrm{H}$ & 0.315584627800 & 6.244099711000 & 6.543111631000 \\
\hline $29 \mathrm{H}$ & 1.205522932000 & 9.105273534000 & 7.371928782000 \\
\hline $30 \mathrm{H}$ & 1.534958935000 & 9.460961551000 & 5.666632718000 \\
\hline
\end{tabular}




\begin{tabular}{|c|c|c|c|}
\hline $31 \mathrm{C}$ & 3.649275831000 & 7.851131814000 & 3.890166538000 \\
\hline $32 \mathrm{H}$ & 4.572695702000 & 7.921815851000 & 3.298413503000 \\
\hline $33 \mathrm{H}$ & 3.237785886000 & 8.868675061000 & 3.999907769000 \\
\hline $34 \mathrm{H}$ & 2.919946384000 & 7.265246917000 & 3.303455898000 \\
\hline \multicolumn{4}{|c|}{ END } \\
\hline \multicolumn{3}{|c|}{ GUIBONDS } & 3434311.0 \\
\hline \multicolumn{3}{|c|}{1151.0} & END \\
\hline \multicolumn{4}{|c|}{21223} \\
\hline \multicolumn{3}{|c|}{3251.0} & CHARGE 0.02 .0 \\
\hline \multicolumn{4}{|c|}{42261.0} \\
\hline \multicolumn{3}{|c|}{52181.0} & UNRESTRICTED \\
\hline \multicolumn{4}{|c|}{62201.0} \\
\hline \multicolumn{3}{|c|}{7361.0} & BASIS \\
\hline \multicolumn{3}{|c|}{83101.0} & type TZP \\
\hline \multicolumn{3}{|c|}{93221.0} & core Large \\
\hline \multicolumn{3}{|c|}{10481.0} & createoutput None \\
\hline \multirow{2}{*}{\multicolumn{3}{|c|}{$\begin{array}{l}114171.0 \\
124221.0\end{array}$}} & END \\
\hline & 1.0 & & \\
\hline \multicolumn{3}{|c|}{135311.0} & $\mathrm{XC}$ \\
\hline \multicolumn{3}{|c|}{14671.0} & GGA Becke Perdew \\
\hline \multicolumn{3}{|c|}{15682.0} & END \\
\hline \multicolumn{4}{|c|}{16891.0} \\
\hline \multicolumn{3}{|c|}{1718301.0} & RELATIVISTIC Scalar ZORA \\
\hline \multicolumn{4}{|c|}{1820251.0} \\
\hline \multicolumn{4}{|c|}{1915171.0} \\
\hline \multicolumn{4}{|c|}{2013101.0} \\
\hline 2120 & 31.0 & & SCANFREQ -1000 0 \\
\hline 2214 & 71.0 & & \\
\hline 2318 & 91.0 & & AnalyticalFreq \\
\hline 2416 & 01.0 & & END \\
\hline 2518 & 41.0 & & \\
\hline 2620 & 11.0 & & SAVE TAPE21 TAPE13 \\
\hline 2712 & 71.0 & & \\
\hline 2811 & 01.0 & & FULLSCF \\
\hline 2926 & 81.0 & & INTEGRATION 6.0 \\
\hline 3026 & 71.0 & & \\
\hline 3126 & 91.0 & & NOPRINT LOGFILE \\
\hline 3232 & 11.0 & & \\
\hline 3333 & 11.0 & & eor \\
\hline$\#==$ & $:============$ & $==========$ & \\
\hline$\# \mathrm{M}$ & $\mathrm{Si} ; \mathrm{X}=\mathrm{Y}=\mathrm{B}(\mathrm{NMe}$ & $\mathrm{H})_{2}$ Singlet & \\
\hline$\#==$ & $=============$ & $==========$ & \\
\hline "\$AL & BIN/adf" <<eor & & \\
\hline ATO & & & \\
\hline $1 \mathrm{Si}$ & 2.774393117000 & 11.212106450000 & 3.372348266000 \\
\hline $2 \mathrm{~B}$ & 2.835078039000 & 9.523133479000 & 4.486019442000 \\
\hline $3 \mathrm{~N}$ & 2.260626722000 & 9.130383924000 & 5.774115325000 \\
\hline $4 \mathrm{C}$ & 2.450540697000 & 7.773576894000 & 5.971349546000 \\
\hline $5 \mathrm{C}$ & 3.134164979000 & 7.252066706000 & 4.902802569000 \\
\hline $6 \mathrm{~N}$ & 3.364710063000 & 8.256073368000 & 3.981699945000 \\
\hline $7 \mathrm{C}$ & 4.063102015000 & 8.007014669000 & 2.728599692000 \\
\hline $8 \mathrm{H}$ & 3.466329122000 & 6.229269826000 & 4.752966091000 \\
\hline
\end{tabular}




\begin{tabular}{|c|c|c|c|}
\hline $9 \mathrm{~N}$ & 3.559330145000 & 12.921412020000 & 5.985128667000 \\
\hline $10 \mathrm{C}$ & 4.415255428000 & 11.998261180000 & 6.717099813000 \\
\hline $11 \mathrm{H}$ & 2.271506903000 & 15.930736220000 & 5.539591837000 \\
\hline $12 \mathrm{C}$ & 3.401580858000 & 14.232520730000 & 6.399909081000 \\
\hline $13 \mathrm{C}$ & 1.498102588000 & 9.929396317000 & 6.723222436000 \\
\hline $14 \mathrm{C}$ & 2.610429124000 & 14.899974980000 & 5.500092486000 \\
\hline $15 \mathrm{~N}$ & 2.273646868000 & 14.040600180000 & 4.471637949000 \\
\hline $16 \mathrm{C}$ & 1.444650204000 & 14.465136860000 & 3.352487787000 \\
\hline $17 \mathrm{H}$ & 2.082186122000 & 7.252972092000 & 6.850279004000 \\
\hline $18 \mathrm{H}$ & 3.866802565000 & 14.621067150000 & 7.300910827000 \\
\hline $19 \mathrm{~B}$ & 2.845838275000 & 12.717893360000 & 4.723047255000 \\
\hline $20 \mathrm{H}$ & 1.902784280000 & 9.818912644000 & 7.740590338000 \\
\hline $21 \mathrm{H}$ & 0.439154683200 & 9.629815017000 & 6.736365210000 \\
\hline $22 \mathrm{H}$ & 1.560650425000 & 10.982387550000 & 6.426639499000 \\
\hline $23 \mathrm{H}$ & 1.781957032000 & 15.439439990000 & 2.970498304000 \\
\hline $24 \mathrm{H}$ & 0.385548903000 & 14.547842950000 & 3.639905393000 \\
\hline $25 \mathrm{H}$ & 1.531316304000 & 13.718444960000 & 2.552510802000 \\
\hline $26 \mathrm{H}$ & 5.471788582000 & 12.300463730000 & 6.656183409000 \\
\hline $27 \mathrm{H}$ & 4.310916146000 & 10.999342420000 & 6.278992460000 \\
\hline $28 \mathrm{H}$ & 4.126838798000 & 11.956255610000 & 7.778251014000 \\
\hline $29 \mathrm{H}$ & 5.146023372000 & 7.887650007000 & 2.884013232000 \\
\hline $30 \mathrm{H}$ & 3.898951106000 & 8.864070099000 & 2.062647526000 \\
\hline $31 \mathrm{H}$ & 3.675512918000 & 7.098805149000 & 2.244935724000 \\
\hline \multicolumn{4}{|l|}{ END } \\
\hline \multicolumn{3}{|c|}{ GUIBONDS } & 313071.0 \\
\hline \multicolumn{3}{|c|}{1123} & 323171.0 \\
\hline \multirow{2}{*}{\multicolumn{3}{|c|}{$\begin{array}{llll}2 & 1 & 19 & 3 \\
3 & 2 & 6 & 1.0\end{array}$}} & END \\
\hline \multirow{2}{*}{\multicolumn{3}{|c|}{$\begin{array}{llll}3 & 2 & 6 & 1.0 \\
4 & 2 & 3 & 1.0\end{array}$}} & \\
\hline & & & BASIS \\
\hline \multicolumn{3}{|c|}{5341.0} & type TZP \\
\hline \multicolumn{3}{|c|}{63131.0} & core Large \\
\hline \multicolumn{3}{|c|}{74171.0} & createoutput None \\
\hline \multicolumn{3}{|c|}{ 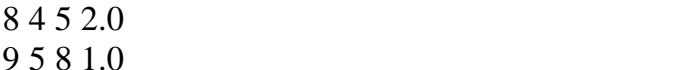 } & END \\
\hline \multicolumn{4}{|c|}{9581.0} \\
\hline \multicolumn{3}{|c|}{10561.0} & $\mathrm{XC}$ \\
\hline \multicolumn{3}{|c|}{11671.0} & GGA Becke Perdew \\
\hline \multicolumn{3}{|c|}{1212142.0} & END \\
\hline \multicolumn{3}{|c|}{1314151.0} & \\
\hline \multicolumn{3}{|c|}{1414111.0} & RELATIVISTIC Scalar ZORA \\
\hline \multicolumn{4}{|c|}{1515161.0} \\
\hline \multicolumn{4}{|c|}{1619151.0} \\
\hline \multicolumn{4}{|c|}{171991.0} \\
\hline \multicolumn{3}{|c|}{189121.0} & SCANFREQ -1000 0 \\
\hline \multicolumn{4}{|c|}{199101.0} \\
\hline 2012 & 81.0 & & AnalyticalFreq \\
\hline 2120 & 31.0 & & END \\
\hline 2221 & 31.0 & & \\
\hline 2322 & 31.0 & & SAVE TAPE21 TAPE13 \\
\hline 2423 & 61.0 & & \\
\hline 2524 & 61.0 & & FULLSCF \\
\hline 2625 & 61.0 & & INTEGRATION 6.0 \\
\hline 2726 & 01.0 & & \\
\hline 2827 & 01.0 & & NOPRINT LOGFILE \\
\hline 2928 & 01.0 & & \\
\hline 3029 & 1.0 & & eor \\
\hline
\end{tabular}




\begin{tabular}{|c|c|c|c|}
\hline \multicolumn{4}{|c|}{ 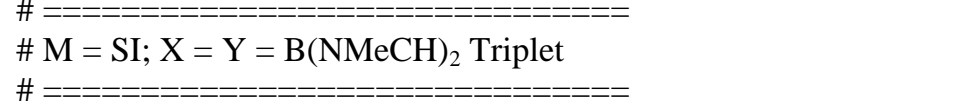 } \\
\hline \multicolumn{4}{|c|}{ "\$ADFBIN/adf" <<eor } \\
\hline $1 \mathrm{Si}$ & 2.800030405000 & 11.190007140000 & 3.665751754000 \\
\hline $2 \mathrm{~B}$ & 2.776764151000 & 9.342393433000 & 4.396346012000 \\
\hline $3 \mathrm{~N}$ & 2.171174173000 & 8.848488156000 & 5.621274450000 \\
\hline $4 \mathrm{C}$ & 2.388227177000 & 7.476830421000 & 5.705176756000 \\
\hline $5 \mathrm{C}$ & 3.079626104000 & 7.060140184000 & 4.601717074000 \\
\hline $6 \mathrm{~N}$ & 3.336445012000 & 8.153282532000 & 3.781367430000 \\
\hline $7 \mathrm{C}$ & 4.058326137000 & 8.025246036000 & 2.526320921000 \\
\hline $8 \mathrm{H}$ & 3.410846265000 & 6.056719740000 & 4.353945663000 \\
\hline $9 \mathrm{~N}$ & 3.633429628000 & 13.225255900000 & 5.871634938000 \\
\hline $10 \mathrm{C}$ & 4.471171822000 & 12.364831210000 & 6.692051936000 \\
\hline $11 \mathrm{H}$ & 2.279492834000 & 16.158269440000 & 5.163876178000 \\
\hline $12 \mathrm{C}$ & 3.432788921000 & 14.567486730000 & 6.177646625000 \\
\hline $13 \mathrm{C}$ & 1.430512844000 & 9.575201158000 & 6.640329795000 \\
\hline $14 \mathrm{C}$ & 2.631600403000 & 15.133482940000 & 5.225221539000 \\
\hline $15 \mathrm{~N}$ & 2.289493889000 & 14.170541210000 & 4.282096579000 \\
\hline $16 \mathrm{C}$ & 1.443575658000 & 14.473652620000 & 3.139587601000 \\
\hline $17 \mathrm{H}$ & 2.040247821000 & 6.883757108000 & 6.545172014000 \\
\hline $18 \mathrm{H}$ & 3.867581788000 & 15.034568810000 & 7.055839016000 \\
\hline $19 \mathrm{~B}$ & 2.904229264000 & 12.910169220000 & 4.654985238000 \\
\hline $20 \mathrm{H}$ & 1.948453867000 & 9.544671903000 & 7.611369637000 \\
\hline $21 \mathrm{H}$ & 0.421996996400 & 9.154461526000 & 6.770095939000 \\
\hline $22 \mathrm{H}$ & 1.333330961000 & 10.621801050000 & 6.329542409000 \\
\hline $23 \mathrm{H}$ & 1.942367878000 & 15.163943080000 & 2.442422900000 \\
\hline $24 \mathrm{H}$ & 0.492886929200 & 14.924769180000 & 3.460772506000 \\
\hline $25 \mathrm{H}$ & 1.223190406000 & 13.540590100000 & 2.607465924000 \\
\hline $26 \mathrm{H}$ & 5.489935437000 & 12.771947610000 & 6.777699318000 \\
\hline $27 \mathrm{H}$ & 4.530193317000 & 11.375811360000 & 6.223268607000 \\
\hline $28 \mathrm{H}$ & 4.056126552000 & 12.248270920000 & 7.705098648000 \\
\hline $29 \mathrm{H}$ & 5.031229384000 & 7.534537513000 & 2.678564077000 \\
\hline $30 \mathrm{H}$ & 4.234827244000 & 9.027422333000 & 2.117888625000 \\
\hline $31 \mathrm{H}$ & 3.483034320000 & 7.444197502000 & 1.789603785000 \\
\hline
\end{tabular}

GUIBONDS

1123

21193

3261.0

4231.0

5341.0

63131.0

74171.0

8452.0

9581.0

10561.0

11671.0

1212142.0

1314151.0
1414111.0

1515161.0

1619151.0

171991.0

189121.0

199101.0

2012181.0

2120131.0

2221131.0

2322131.0

2423161.0

2524161.0

2625161.0

2726101.0 
2827101.0

2928101.0

302971.0

313071.0

323171.0

END

CHARGE 0.02 .0

UNRESTRICTED

BASIS
type TZP
core Large
createoutput None
END
XC
GGA Becke Perdew

END

RELATIVISTIC Scalar ZORA

SCANFREQ -1000 0

AnalyticalFreq

END

SAVE TAPE21 TAPE13

FULLSCF

INTEGRATION 6.0

NOPRINT LOGFILE

eor

\begin{tabular}{|c|c|c|c|}
\hline \multicolumn{4}{|c|}{ 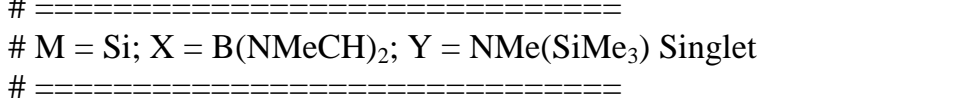 } \\
\hline \multicolumn{4}{|c|}{ "\$ADFBIN/adf" <<eor } \\
\hline $1 \mathrm{Si}$ & 5.458754548000 & 6.962516607000 & 4.417095623000 \\
\hline $2 \mathrm{Si}$ & 3.209183708000 & 7.063517243000 & 6.712310783000 \\
\hline $3 \mathrm{~N}$ & 7.285719420000 & 5.656882316000 & 6.653346341000 \\
\hline $4 \mathrm{~N}$ & 7.437941209000 & 7.925985652000 & 6.691410529000 \\
\hline $5 \mathrm{~N}$ & 3.808194473000 & 7.057202680000 & 5.010776975000 \\
\hline $6 \mathrm{C}$ & 8.310729129000 & 6.030307048000 & 7.525739765000 \\
\hline $7 \mathrm{H}$ & 8.900830235000 & 5.303299976000 & 8.075316871000 \\
\hline $8 \mathrm{C}$ & 8.401914125000 & 7.390323400000 & 7.548689202000 \\
\hline $9 \mathrm{H}$ & 9.082132928000 & 8.013353264000 & 8.121325354000 \\
\hline $10 \mathrm{C}$ & 7.008513319000 & 4.257586736000 & 6.368623446000 \\
\hline $11 \mathrm{H}$ & 6.720454933000 & 3.709852135000 & 7.279434141000 \\
\hline $12 \mathrm{H}$ & 6.541074028000 & 9.560336212000 & 5.742691214000 \\
\hline $13 \mathrm{H}$ & 7.885176254000 & 3.759856790000 & 5.926206271000 \\
\hline $14 \mathrm{H}$ & 7.124860653000 & 9.907131575000 & 7.384721134000 \\
\hline $15 \mathrm{H}$ & 8.286432050000 & 9.752868395000 & 6.036636714000 \\
\hline $16 \mathrm{H}$ & 6.178277818000 & 4.188777222000 & 5.656583430000 \\
\hline $17 \mathrm{C}$ & 7.348097562000 & 9.358688646000 & 6.456298044000 \\
\hline $18 \mathrm{C}$ & 1.313726914000 & 7.185394917000 & 6.708721320000 \\
\hline $19 \mathrm{H}$ & 4.771013878000 & 5.376237552000 & 7.710496196000 \\
\hline $20 \mathrm{C}$ & 3.882568431000 & 8.556640114000 & 7.652029841000 \\
\hline $21 \mathrm{H}$ & 4.974576455000 & 8.497511710000 & 7.764502888000 \\
\hline $22 \mathrm{~B}$ & 6.674338594000 & 6.847289282000 & 6.092407972000 \\
\hline $23 \mathrm{H}$ & 3.439008786000 & 8.594669250000 & 8.660482493000 \\
\hline $24 \mathrm{H}$ & 0.946321657600 & 8.109404806000 & 6.236560264000 \\
\hline $25 \mathrm{H}$ & 3.633614514000 & 9.498005590000 & 7.137910854000 \\
\hline $26 \mathrm{C}$ & 3.681127004000 & 5.462616844000 & 7.595681116000 \\
\hline $27 \mathrm{H}$ & 3.315057024000 & 4.580472784000 & 7.047756559000 \\
\hline $28 \mathrm{H}$ & 3.231528960000 & 5.445921820000 & 8.602083059000 \\
\hline $29 \mathrm{H}$ & 0.962906645000 & 7.189702046000 & 7.753966003000 \\
\hline
\end{tabular}




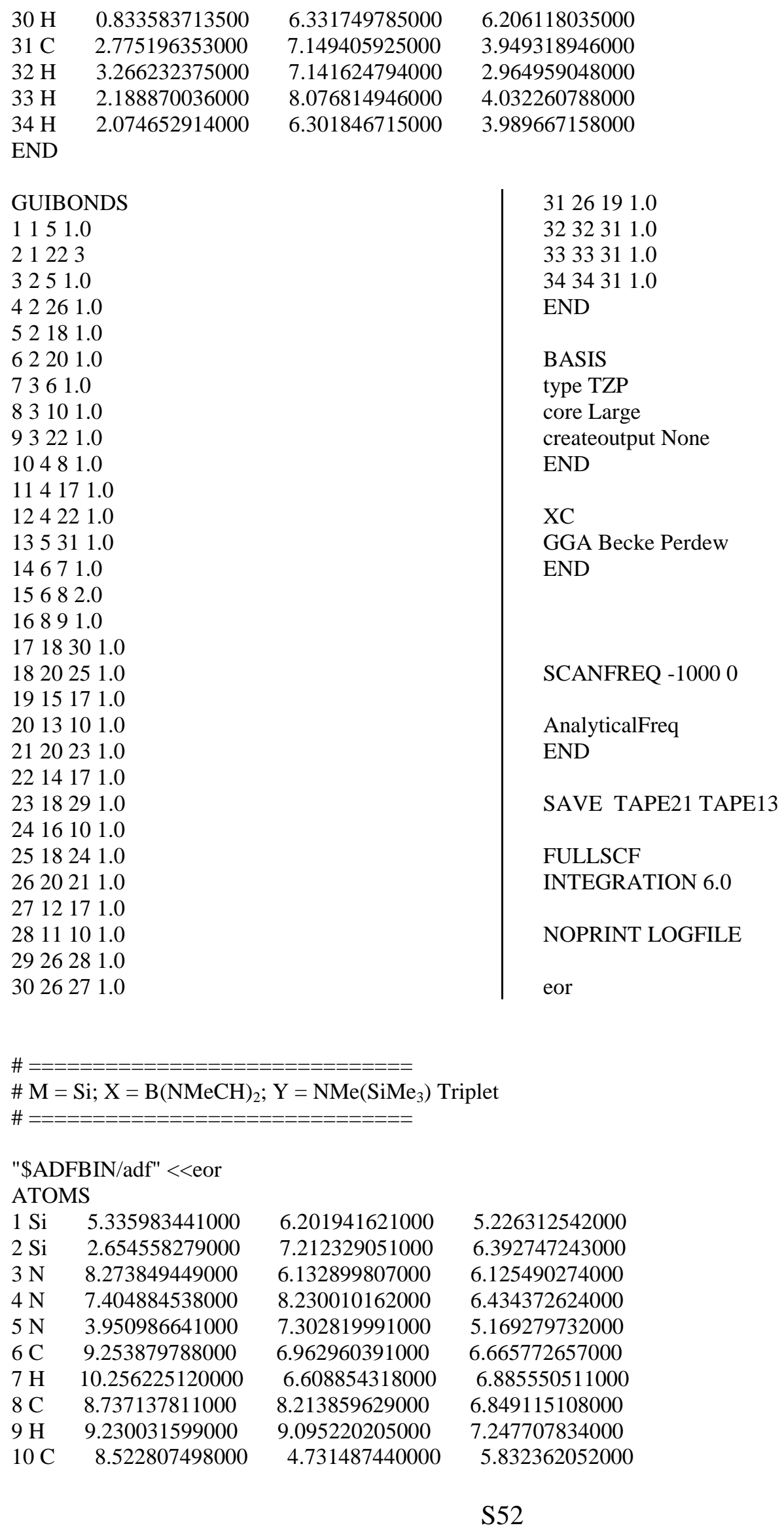




$\begin{array}{lccc}11 \mathrm{H} & 8.774900299000 & 4.170671063000 & 6.745666375000 \\ 12 \mathrm{H} & 5.586718935000 & 9.193903292000 & 6.130788943000 \\ 13 \mathrm{H} & 9.345685760000 & 4.615366200000 & 5.110615105000 \\ 14 \mathrm{H} & 6.509918791000 & 9.804202872000 & 7.530524400000 \\ 15 \mathrm{H} & 7.013638902000 & 10.233228730000 & 5.871699740000 \\ 16 \mathrm{H} & 7.619191424000 & 4.288710431000 & 5.396421629000 \\ 17 \mathrm{C} & 6.590723848000 & 9.431783292000 & 6.497331630000 \\ 18 \mathrm{C} & 1.799864544000 & 8.903683960000 & 6.497712816000 \\ 19 \mathrm{H} & 1.835963423000 & 4.907836259000 & 5.847512094000 \\ 20 \mathrm{C} & 3.408026847000 & 6.787877395000 & 8.069243426000 \\ 21 \mathrm{H} & 3.869037148000 & 5.789090968000 & 8.073133362000 \\ 22 \mathrm{~B} & 7.059680746000 & 6.905517472000 & 5.958794584000 \\ 23 \mathrm{H} & 2.620737957000 & 6.802847577000 & 8.841275124000 \\ 24 \mathrm{H} & 2.516237686000 & 9.707901265000 & 6.727651661000 \\ 25 \mathrm{H} & 4.182397639000 & 7.513514955000 & 8.359813659000 \\ 26 \mathrm{C} & 1.372481689000 & 5.905028738000 & 5.904100307000 \\ 27 \mathrm{H} & 0.920843985300 & 6.123585796000 & 4.923703387000 \\ 28 \mathrm{H} & 0.555466934300 & 5.857926541000 & 6.642145968000 \\ 29 \mathrm{H} & 1.049092803000 & 8.886789653000 & 7.304453119000 \\ 30 \mathrm{H} & 1.273560107000 & 9.170134020000 & 5.568406825000 \\ 31 \mathrm{C} & 3.603946996000 & 7.923221865000 & 3.868639891000 \\ 32 \mathrm{H} & 4.495799202000 & 7.989184765000 & 3.230265351000 \\ 33 \mathrm{H} & 3.215736963000 & 8.942432255000 & 4.019361726000 \\ 34 \mathrm{H} & 2.840685381000 & 7.348533416000 & 3.317810203000 \\ \mathrm{END} & & & \end{array}$




\section{GUIBONDS}

1151.0

21223

3251.0

42261.0

52181.0

62201.0

7361.0

83101.0

93221.0

10481.0

114171.0

124221.0

135311.0

14671.0

15682.0

16891.0

1718301.0

1820251.0

1915171.0

2013101.0

2120231.0

2214171.0

2318291.0

2416101.0

2518241.0

2620211.0

2712171.0

2811101.0

2926281.0

3026271.0

3126191.0

3232311.0

3333311.0

3434311.0

END

CHARGE 0.02 .0

UNRESTRICTED

\section{BASIS}

type TZP

core Large

createoutput None

END

$\mathrm{XC}$

GGA Becke Perdew

END

SCANFREQ -1000 0

AnalyticalFreq

END

SAVE TAPE21 TAPE13

Page S54 
FULLSCF

INTEGRATION 6.0

NOPRINT LOGFILE

eor

Page S55 